\title{
Conjuntos fortemente nulos e fortemente magros
}

\author{
Guilherme Trajano de Santana \\ DISSERTAÇÃO APRESENTADA \\ $\mathrm{AO}$ \\ Instituto De MatemáticA e EstatísticA \\ DA \\ Universidade DE SÃo PAUlo \\ PARA \\ OBTENÇÃO DO TÍTULO \\ DE \\ Mestre EM CiÊnCIAS \\ Programa: Matemática \\ Orientador: Prof. Dr. Rodrigo Roque Dias
}

Durante o desenvolvimento deste trabalho o autor recebeu auxílio financeiro da CNPq

São Paulo, abril de 2019 


\section{Conjuntos fortemente nulos e fortemente magros}

Esta versão da dissertação/tese contém as correções e alterações sugeridas pela Comissão Julgadora durante a defesa da versão original do trabalho, realizada em 18/03/2019. Uma cópia da versão original está disponível no Instituto de Matemática e Estatística da Universidade de São Paulo.

Comissão Julgadora:

- Prof. Dr. Rodrigo Roque Dias - IME - USP

- Prof $^{\mathrm{a}}$. Dra . Ana Carolina Boero - UFABC

- Prof. Dr. Leandro Fiorini Aurichi - ICMC - USP 


\section{Resumo}

Santana, G. T. Conjuntos fortemente nulos e fortemente magros. 2019. $120 \mathrm{f}$. Dissertação - Instituto de Matemática e Estatística, Universidade de São Paulo, São Paulo, 2019.

O presente trabalho tem como objetivo apresentar os conjuntos fortemente nulos e fortemente magros. Mais especificamente, iremos apresentar algumas aplicações e avaliar a independência de ZFC de afirmações envolvendo tais conjuntos. Com relação às aplicações, daremos alguns exemplos de conjuntos fortemente nulos e fortemente magros, estudaremos a aditividade do ideal formado pelos subconjuntos fortemente nulos da reta real, apresentaremos uma análise da relação entre a propriedade fortemente nulo e translações de subconjuntos da reta, mostraremos equivalências da Conjectura de Borel em espaços métricos, com a afirmação R-BC e com uma afirmação envolvendo jogos. Com relação a análise de independência de afirmações de ZFC, mostraremos que a Conjectura Dual de Borel é independente de ZFC e que a negação da Conjectura de Borel é consistente com ZFC.

Palavras-chave: Conjuntos fortemente nulos; Conjuntos fortemente magros; Conjectura de Borel; Conjectura Dual de Borel; Dualidade. 


\section{Abstract}

Santana, G. T. Strongly null and strongly meager sets. 2019. 120 f. Dissertação - Instituto de Matemática e Estatística, Universidade de São Paulo, São Paulo, 2019. The present work aims to present the strongly null and strongly meager sets. More specifically, we will present some applications and evaluate the independence of ZFC from statements involving such sets. With respect to the applications, we will give some examples of strongly null and strongly meager sets, we will study the additivity of the ideal formed by the strongly null subsets of the real line, we will present an analysis of the relation between the strongly null property and the subsets of the line, of the Borel Conjecture in metric spaces, with the statement R-BC and with a statement involving games. Regarding the analysis of the independence of ZFC statements, we will show that the Borel Dual Conjecture is independent of ZFC and that the negation of the Borel Conjecture is consistent with ZFC.

Keywords: Strongly null; Strongly meager; Borel conjecture; Dual Borel conjecture; Duality. 


\section{Conteúdo}

\begin{tabular}{lll}
\hline 1 & Introdução & 7
\end{tabular}

\begin{tabular}{lll}
\hline 2 & Preliminares & 11
\end{tabular}

$2.1 \quad$ O Espaço $\mathbb{K}$, Homeomorfismos e Conjuntos de Cantor . . . . . . . . . . 11

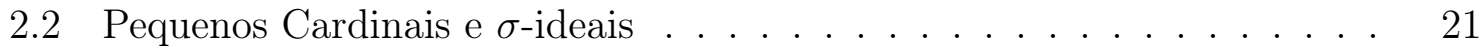

2.3 Forcing: alguns resultados e comentários . . . . . . . . . . . . . . . . . 31

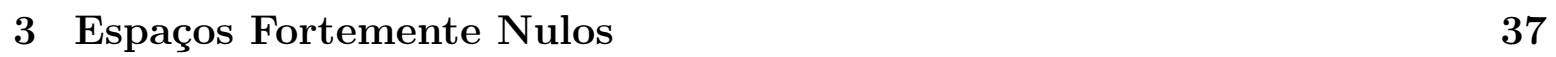

3.1 Definições, Exemplos e Resultados Básicos . . . . . . . . . . . . . . . . 37

$3.2 \quad$ Aditividade de $\mathcal{S N}$. . . . . . . . . . . . . . . . . . . . . . . . . . . . . . . 48

3.3 Translações . . . . . . . . . . . . . . . . . . . . . . . . . . . . 53

3.4 Conjectura de Borel . . . . . . . . . . . . . . . . . . . . 62

3.5 Teorema de Goldstern-Judah-Shelah . . . . . . . . . . . . . . . . . . . 64

$\begin{array}{lll}4 & \text { Equivalências } & 69\end{array}$

4.1 Espaços Métricos e a Conjectura de Borel . . . . . . . . . . . . . . . . 69

$4.2 \quad$ Propriedade de Rothberger e a Conjectura de Borel em $\mathbb{R}$. . . . . . . . 74

4.3 Jogos . . . . . . . . . . . . . . . . . . . . . . . 78

5 Espaços Fortemente Magros $\quad 83$

5.1 Teorema de Erdős-Sierpiński . . . . . . . . . . . . . . . . . . . . . . . 83

5.2 Conjectura Dual de Borel . . . . . . . . . . . . . . . . . . . . . . 91 
CONTEÚDO 


\section{Capítulo 1}

\section{Introdução}

Borel em 1919 (4]) introduziu o conceito de conjunto fortemente nulo na reta real (Definição 3.1.1). Iremos demonstrar que tal conceito estende de maneira natural a definição de conjunto de medida nula que estudamos nos cursos de análise com a Proposição 3.1.4 desenvolvida na Seção 3.1. Veremos que tal extensão, é de fato, estrita, uma vez que iremos apresentar uma demonstração de que o Conjunto de Cantor $2^{\omega}$ não é fortemente nulo na mesma seção (Teorema 3.1.11). Adicionalmente, veremos na Seção 3.1 que a propriedade fortemente nulo não é preservada por funções contínuas. Apresentaremos uma demonstração de que assumindo-se a Hipótese do Contínuo existe um subconjunto do intervalo $[0,1]$ e função contínua que mapeia tal conjunto no conjunto de Cantor, $2^{\omega}$ (Teorema 3.1.18). Como veremos na Proposição 3.1.13, se temos um conjunto fortemente nulo que é subconjunto do domínio de uma função uniformemente contínua então a imagem do conjunto fortemente nulo pela função uniformemente contínua também é fortemente nulo.

Pode-se demonstrar facilmente que todo subconjunto enumerável da reta real é fortemente nulo. A recíproca dessa afirmação conhecida como Conjectura de Borel é uma afirmação independente de ZFC, apresentaremos o enunciado formal de tal conjectura na Seção 3.4. Assumindo a Hipótese do Contínuo iremos apresentar a demonstração da existência de um conjunto de Luzin. Sierpiński ([15]) demonstrou que todo conjunto de Luzin é fortemente nulo, como veremos no Teorema 3.4.3. Apresentaremos uma demonstração alternativa da consistência da negação da Conjectura de Borel, Goldestern ([8]) utilizando conceitos combinatórios também demonstrou a consistência da negação da Conjectura de Borel (Teorema 3.5.7). Adicionalmente, exibiremos duas consequên- 
cias do Teorema de Goldstern, a primeira é a apresentação de um conjunto fortemente nulo de cardinalidade $\mathfrak{b}$ e a segunda é a apresentação de um conjunto fortemente nulo de cardinalidade $\mathfrak{d}$. Não apresentaremos uma prova de que a Conjectura de Borel é consistênte com ZFC, mas Laver ([12]) usando a técnica de forcing demonstrou a consistência da Conjectura de Borel.

$\mathrm{Na}$ Seção 3.1 apresentaremos uma prova de que os subconjuntos da reta real que são fortemente nulos constituem um $\sigma$-ideal (Teorema 3.1.5). Sabemos que assumindo-se o Axioma de Martin o $\sigma$-ideal dos subconjuntos da reta real real que possuem medida de Lebesgue igual a zero é fechado por reuniões de subfamílias com cardinalidade estritamente menor que contínuo. O resultado análogo também é válido na teoria dos conjuntos fortemente nulos, Carlson (5]) provou que assumindo-se o Axioma de Martin tal $\sigma$-ideal é fechado por reuniões de subfamílias com cardinalidade estritamente menor que contínuo, como veremos no Corolário 3.2.12. Exibiremos na Seção 3.1 a definição da propriedade de Rothberger ([14]), uma propriedade topológica associada ao estudo de conjuntos fortemente nulos (Definição 3.1.14). Formularemos uma afirmação na Seção 4.2, que nos diz que todo subconjunto da reta real que possui a propriedade de Rothberger é enumerável. Na mesma seção exibiremos uma demonstração de que tal afirmativa é equivalente à Conjectura de Borel (Miller ([2])) (Corolário 4.2.13).

Na Seção 4.3 que é referente a jogos topológicos, apresentaremos um jogo (Definição 4.3.7) introduzido por Galvin-Mycielski-Solovay ([7]). No decorrer iremos trabalhar para demonstrarmos que o jogo introduzido é determinado se, e somente se, vale a Conjectura de Borel (Corolário 4.3.14). Uma noção equivalente da propriedade fortemente nula envolvendo translações é apresentada na Seção 3.3, como veremos no Corolário 3.3.19. O Teorema de Erdôs-Sierpiński (Teorema 5.1.16) expressa uma dualidade existente entre conjuntos de medida nula e conjuntos magros da reta real. Tal noção sugere uma definição dual a de conjunto fortemente nulo, o conceito de conjunto fortemente magro (Definição 5.2.1). Que de forma análoga, também estende a definição de subconjunto magro (Proposição 5.2.3) da reta real. Tendo em mente essa dualidade entre conjuntos fortemente nulos e conjuntos fortemente magros, Carlson ([5]) enuncia a Conjectura Dual de Borel que é a afirmação que todo subconjunto fortemente magro é enumerável. Na Seção 5.2 iremos apresentar uma demonstração devida a Carlson (5]), de que usando a técnica de forcing, é possível construir um modelo transitivo enumerável de ZFC que satisfaz a Conjectura Dual de Borel. Demonstrando, então a 
consistência da Conjectura Dual de Borel (Teorema 5.2.25). Fazendo uso de pequenos cardinais que iremos apresentar na Seção 2.2 iremos apresentar uma demonstração de que existe um subconjunto fortemente nulo da reta real que possui cardinalidade igual a $\operatorname{cof}(\mathcal{N}) \geq \aleph_{1}$, ou seja, a Conjectura Dual de Borel é uma afirmação também independente de ZFC (Teorema 5.2.5). 


\section{Capítulo 2}

\section{Preliminares}

\subsection{O Espaço $\mathbb{K}$, Homeomorfismos e Conjuntos de Can- tor}

O objetivo dessa seção é introduzir e analisar o espaço $\mathbb{K}$. Apresentar uma demonstração que o espaço de Baire é homeomorfo aos irracionais. Adicionalmente, realizaremos construções de conjuntos de Cantor.

Ao longo desse trabalho denotaremos por $\mathbb{K}$ o conjunto $[0,1[\subseteq \mathbb{R}$ munido da métrica $d:[0,1[\times[0,1[\longrightarrow \mathbb{R}$ dada por $d(x, y)=\min \{|x-y|, 1-|x-y|\}$.

A medida em $\mathbb{K}$ é a medida de Lebesgue usual da reta real restrita a $\mathbb{K}$. Logo, os conjuntos de medida zero de $\mathbb{K}$ são os conjuntos de medida zero de $[0,1]$.

Sejam $a, b \in \mathbb{K}$. Sempre estaremos nos referindo ao intervalo $[a, b]$ (analogamente a outros tipos de intervalos, por exemplo: $[a, b[] a,, b[\ldots)$ em $\mathbb{K}$ da seguinte forma:

$$
[a, b]= \begin{cases}\{x \in[0,1[: a<x<1 \text { ou } 0 \leq x<b\}, & \text { se } b<a \\ \{x \in[0,1[: a \leq x \text { e } x \leq b\}, & \text { se } a \leq b\end{cases}
$$

Considere $x, y \in \mathbb{K}$. Definiremos $x+y$ como sendo:

$$
\left\{\begin{array}{lr}
x+y, & \text { se } x+y<1 \\
x+y-1, & \text { caso contrário }
\end{array}\right.
$$

O oposto de $x$ denotado por $-x$ é definido por: 


$$
\left\{\begin{array}{lr}
0, & \text { se } x=0 ; \\
1-x, & \text { caso contrário } ;
\end{array}\right.
$$

Por conseguinte, $x-y=x+(-y)$. Observe que $x-x=0$.

Abaixo apresentamos a noção de translação em $\mathbb{K}$ e o conceito de soma de conjuntos de $\mathbb{K}$.

Definição 2.1.1. Sejam $A, B \subseteq \mathbb{K}$ e $x \in \mathbb{R}$. Definimos:

(i) $A+x=\{a+x: a \in A\}$;

(ii) $A+B=\{a+b: a \in A$ e $b \in B\}$.

Considere $f: \mathbb{K} \longrightarrow \mathbb{S}^{1}$ dada por $f(x)=(\sin (2 \cdot \pi \cdot x), \cos (2 \cdot \pi \cdot x))$ para cada $x \in \mathbb{K}$. Observe que $f$ é um homeomorfismo. Como $\mathbb{S}^{1}$ é compacto e a compacidade é uma propriedade topológica então $\mathbb{K}$ é um espaço métrico compacto.

As translações de intervalos de $\mathbb{K}$ se comportam de maneira semelhante as translações de intervalos sobre $\mathbb{S}^{1}$. O lema abaixo nos mostra uma maneira de somar intervalos em $\mathbb{K}$.

Observação 2.1.2. Para cada $x, y \in \mathbb{K} e \varepsilon \in \mathbb{R}$ temos que:

$$
] x-\varepsilon, x+\varepsilon[+] y-\varepsilon, y+\varepsilon[=](x+y)-2 \varepsilon,(x+y)+2 \varepsilon[.
$$

Uma propriedade relativa a translações é enunciada abaixo. Será utilizada na Seção 3.3 .

Observação 2.1.3. Sejam $\left.\delta_{0} \in\right] 0,1[$ e $x, y \in \mathbb{K}$. Então temos:

$$
] 0, \delta_{0}[+(x+y)=] x+y, \delta_{0}+(x+y)[.
$$

Apresentamos a seguir definição de distância de um ponto a um conjunto em um espaço métrico arbitrário.

Definição 2.1.4. Seja $(X, d)$ um espaço métrico. Considere $a \in X$ e $A \subseteq X$ um subconjunto não vazio. Definimos a distância do ponto a ao conjunto $A$ como sendo $d(a, A)=\inf \left\{d\left(a, a^{\prime}\right): a^{\prime} \in A\right\}$. 
Segue abaixo o conceito de continuidade uniforme.

Definição 2.1.5. Sejam $\left(X, d_{X}\right)$ e $\left(Y, d_{Y}\right)$ espaços métricos. Dizemos que uma função $f: X \rightarrow Y$ é uniformemente contínua se, para cada $\varepsilon>0$, existe $\delta>0$ tal que, para quaisquer $x, y \in X \operatorname{com} d_{X}(x, y)<\delta$, temos $d_{Y}(f(x), f(y))<\varepsilon$.

Lema 2.1.6. Seja $(X, d)$ um espaço métrico. Considere $x, y \in X$ e $D \subseteq X$ um subconjunto não vazio. Então, vale a desigualdade:

$$
|d(x, D)-d(y, D)| \leq d(x, y) .
$$

Demonstração. Sejam $x, y \in X$ e $D \subseteq X$ um subconjunto não vazio. Devemos mostrar que

$$
-d(x, y) \leq d(x, D)-d(y, D) \leq d(x, y)
$$

De fato, para cada $z \in D$ temos

$$
\begin{aligned}
& d(x, D) \leq d(x, z) \leq d(x, y)+d(y, z), \text { ou seja, } \\
& d(x, D)-d(x, y) \leq d(y, z) . \text { Como vale para cada } z \in D, \\
& d(x, D)-d(x, y) \leq d(y, D) \text { e, portanto, } \\
& d(x, D)-d(y, D) \leq d(x, y)
\end{aligned}
$$

A desigualdade é uma das desigualdades que deveríamos demonstrar. A outra segue de $\mathbf{s}$ trocando-se $x$ por $y$ e multiplicando por -1 .

O Lema 2.1.7 nos mostra que a função que calcula a distância de um ponto a um conjunto fixado em um espaço mético é uniformemente contínua.

Lema 2.1.7. Sejam $(X, d)$ um espaço métrico e $D \subseteq X$ um subconjunto não vazio. Então, $f: X \longrightarrow \mathbb{R}$ dada por $f(x)=d(x, D)$ é uma função uniformemente contínua.

Demonstração. Seja $\varepsilon>0$ arbitrário. Tome $\delta=\varepsilon$. Sejam $x, y \in X$, tais que $d(x, y)<\delta$. Pelo lema anterior, temos:

$$
|f(x)-f(y)|=|d(x, D)-d(y, D)| \leq d(x, y)<\varepsilon .
$$

Logo, $f$ é uniformemente contínua. 
Definição 2.1.8. Chamamos de Espaço de Baire o espaço das funções de $\omega \mathrm{em} \omega$. Tal espaço é denotado por $\omega^{\omega}$.

Denotaremos por $\omega^{<\omega}$ o conjunto das sequências finitas com elementos em $\omega$ e por () a sequência vazia, ou seja, a sequência que não possui elementos.

Se $s \in \omega^{<\omega}$ então $|s|$ denota o comprimento de $s$, isto é, a cardinalidade de $\operatorname{dom}(s)$. Se $n \in \omega$ então denotaremos por $s^{\frown} n$ a sequência $s$ concatenada com $n$, por exemplo, $(0127) \frown 3=(01273)$.

Os abertos básicos de $\omega^{\omega}$ são conjuntos da forma:

$$
[s]=\left\{x \in \omega^{\omega}: s \subseteq x\right\}
$$

para cada $s \in \omega^{\omega}$. Um conjunto é aberto em $\omega^{\omega}$ se, e somente se, é a reunião de abertos básicos.

O Lema 2.2 .12 nos ajuda a demonstrar que o espaço de Baire é homeomorfo aos irracionais.

Definição 2.1.9. Considere $I \subseteq \mathbb{R}$ um intervalo de $\mathbb{R}$. Sejam $a$ o extremo esquerdo do intervalo $I$ e $b$ o extremo direito do intervalo $I$. Definimos $\ell(I)$ como sendo $b-a$.

Lema 2.1.10. Se $\left\{q_{n}: n \in \omega\right\}$ é uma enumeração de $\mathbb{Q}$ sem repetições então existe uma sequência $\left(I_{s}\right)_{s \in \mathbb{Z}<\omega}$ de intervalos abertos em $\mathbb{R}$ que satisfaz:

1. $I_{()}=\mathbb{R}$ e $I_{s} \neq \emptyset$ é um intervalo aberto com extremos racionais para cada $s \in \mathbb{Z}^{<\omega}$ tal que $s \neq \emptyset$;

2. $I_{s}{ }_{n} \subseteq I_{s}$ para cada $s \in \mathbb{Z}^{<\omega}$ e $n \in \mathbb{Z}$;

3. o extremo direito do intervalo $I_{s} \neg_{n}$ é o extremo esquerdo do intervalo $I_{s} \smile_{(n+1)}$; 4. $\forall s \in \mathbb{Z}^{<\omega}, \bigcup_{n \in \mathbb{Z}} I_{s{ }_{n}}=I_{s} \backslash \bigcup_{n \in \mathbb{Z}}\left\{a_{s{ }_{n}}\right\}$;

5. $\ell\left(I_{s}\right) \leq \frac{1}{|s|}$ para cada $s \neq()$

6. $q_{n}$ é ponto extremo de $I_{t}$ para algum $t \in \mathbb{Z}^{<\omega}$ tal que $|t| \leq n+1$.

Demonstração. Iremos demonstrar o resultado por indução no comprimento da sequência. Primeiramente, para a sequência vazia ()$\in \mathbb{Z}^{<\omega}$ defina $I_{()}=\mathbb{R}$.

Fixe $s \in \mathbb{Z}$ tal que $|s|=1$. Então, existe $m \in \mathbb{Z}$ tal que $s=(m)$ e portanto $I_{s}=I_{(m)}$. Defina $\left.I_{(m)}=\right] q_{0}+m, q_{0}+(m+1)\left[\right.$. Note que $\left(I_{m}\right)_{m \in \mathbb{Z}}$ cumpre as condições de 1 . a 6 .. 
Suponha que o resultado vale para cada $t \in \mathbb{Z}^{<\omega}$ tal que $|t|=n$. Vamos estudar o caso em que $t \in \mathbb{Z}$ e $|t|=n+1$.

Fixe $s \in \mathbb{Z}^{<\omega}$ tal que $|s|=n+1$. Note que $s$ é uma sequência da forma $t^{\frown}(a)$, onde $t \in \mathbb{Z}^{<\omega}$ possui comprimento $n$ e $a \in \mathbb{Z}$.

Considere o intervalo aberto não vazio de extremos racionais $I_{t}$, dado pela hipótese de indução. Devemos definir $I_{t}{ }_{(a)}$. Seja $\xi_{t}$ o ponto médio do intervalo $I_{t}$.

Vamos analisar dois casos:

$\circ q_{n} \notin I_{t}$ ou $q_{n}=\xi_{t}$ : Sejam $\left(x_{k}^{t}\right)_{k \in \omega}$ e $\left(y_{m}^{t}\right)_{m \in \omega}$ sequências de números racionais tais que $\left.x_{k}^{t} \in\right] a_{t}, \xi_{t}\left[\right.$ para cada $\left.k \in \omega, y_{m}^{t} \in\right] \xi_{t}, b_{t}\left[\right.$ para cada $m \in \omega, x_{k}^{t}>x_{k+1}^{t}$ para cada $t \in \omega, y_{m}^{t}<y_{m+1}^{t}$ para cada $m \in \omega, x_{k}^{t} \stackrel{k \rightarrow \infty}{\longrightarrow} a_{t}$ e $y_{m}^{t} \stackrel{m \rightarrow \infty}{\longrightarrow} b_{t}$. Defina $\left.I_{t}{ }_{(0)}=\right] \xi_{t}, y_{0}^{t}[$,

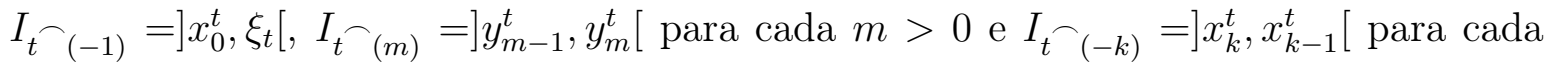
$k>0$.

$\circ q_{n} \in I_{t} \backslash\left\{\xi_{t}\right\}$ : Suponhamos que $q_{n}<\xi_{t}$ (a situação $q_{n}>\xi_{t}$ é análoga). Nesse caso, defina $\left.I_{t \frown 0}=\right] q_{n}, \xi_{t}\left[, I_{t \frown(-1)}=\right] \xi_{t}, y_{0}^{t}\left[, I_{t \frown(-1)}=\right] x_{0}^{t}, q_{n}\left[, I_{t \frown(m)}=\right] y_{m-1}^{t}, y_{m}^{t}[$ para $m>0 \mathrm{e}$ $\left.I_{t-(-k)}=\right] x_{k}^{t}, x_{k-1}^{t}[$ para $k>0$.

Antes de mostrarmos que o espaço de Baire é homeomorfo aos irracionais precisaremos de uma lema auxiliar que apresentamos abaixo.

Definição 2.1.11. Considere $(X, d)$ um espaço métrico e $\mathrm{Y}$ um subconjunto de $\mathrm{X}$. Definimos $\operatorname{diam}(Y)$ como sendo $\sup \{d(x, y): x, y \in Y\}$.

Lema 2.1.12. $\mathbb{Z}^{\omega}$ é homeomorfo a $\mathbb{P}$.

Demonstração. Fixe $x \in \mathbb{Z}^{\omega}$. Queremos definir $f(x)$, para tanto considere a sequência $\left(I_{s}\right)_{s \in \mathbb{Z}<\omega}$ dada pelo Lema 2.2.12.

Pelo Lema 2.2 .12 temos que $\overline{I_{\left.x\right|_{n}}} \subseteq \overline{I_{x \uparrow_{(n+1)}}}$ para cada $n \in \omega$ e que diam $\left(\overline{I_{x \uparrow n}}\right) \stackrel{n \rightarrow \infty}{\longrightarrow} 0$. Logo, existe $p \in \mathbb{R}$ tal que $\{p\}=\bigcap_{n \in \omega} I_{x\lceil n}$. Pelo Lema 2.2.12 temos que $p \in \mathbb{P}$. Defina $f(x)=p$.

A função $f: \mathbb{Z}^{\omega} \longrightarrow \mathbb{P}$ é injetora, pois $I_{s} \cap I_{t}=\emptyset$ se $s, t \in \mathbb{Z}^{<\omega}$ são incomparáveis. A função $f$ é sobrejetora, pois para cada $p \in \mathbb{P}$ e $n \in \omega$ existe um único $s \in \mathbb{Z}^{<\omega}$ tal que $\ell(s)=n$ com $p \in I_{s}$.

Note que, $\left\{I_{s} \cap \mathbb{P}: s \in \mathbb{Z}^{<\omega}\right\}$ é uma base para a topologia usual de $\mathbb{P}$. Note que $f([s])=I_{s} \cap \mathbb{P}$ para cada $s \in \mathbb{Z}^{<\omega}$. Como $f$ é contínua, bijetora e leva aberto básico em aberto básico, temos que $f$ é um homeomorfismo. 
Definição 2.1.13. Denotaremos por $\mathbb{P}$ o espaço $\mathbb{R} \backslash \mathbb{Q}$ com a topologia euclidiana.

Abaixo apresentamos a demonstração de que o espaço de Baire é homeomorfo aos irracionais.

Teorema 2.1.14. $\omega^{\omega}$ é homeomorfo a $\mathbb{P}$.

Demonstração. Sabemos que $\omega$ é homeomorfo a $\mathbb{Z}$ e, portanto temos que $\omega^{\omega}$ é homeomorfo a $\mathbb{Z}^{\omega}$. Pelo Lema 2.1 .12 temos que $\mathbb{Z}^{\omega}$ é homeomorfo a $\mathbb{P}$. Portanto, $\omega^{\omega}$ é homeomorfo a $\mathbb{P}$.

De maneira análoga também podemos demonstrar que o Espaço de Baire é homeomorfo aos irracionais em $[0,1]$.

Abaixo realizamos a definição de conjunto de Cantor.

Definição 2.1.15. Seja $X$ um espaço topológico. Dizemos que $X$ é um Conjunto de Cantor se $X$ é homeomorfo ao produto topológico $2^{\omega}$, sendo que $2=\{0,1\}$ possui a topologia discreta.

A estrutura definida abaixo nos ajuda a realizar construções de conjuntos de Cantor.

Definição 2.1.16. Seja $(X, d)$ um espaço métrico. Dizemos que uma sequência $\left(F_{s}\right)_{s \in 2<\omega}$ é uma estrutura do tipo árvore em $X$ se:

(1) $F_{s} \subseteq X$ é um fechado não vazio de $X$ para cada $s \in 2^{<\omega}$;

(2) $F_{s{ }_{0}}, F_{s} \frown_{1} \subseteq F_{s}$ e $F_{s} \frown_{0} \cap F_{s} \frown_{1}=\emptyset$ para cada $s \in 2^{<\omega}$;

(3) Para cada $f \in 2^{\omega}$ tem-se $\operatorname{diam}\left(F_{f \nmid n}\right) \stackrel{n \rightarrow \infty}{\longrightarrow} 0$.

Abaixo exibimos nossa primeira construção relativa a conjuntos de Cantor.

Teorema 2.1.17. Sejam $(X, d)$ um espaço métrico completo e $\left(F_{s}\right)_{s \in 2<\omega}$ uma estrutura do tipo árvore em $X$. Se $C_{n}=\bigcup\left\{F_{s}: s \in 2^{<\omega}\right.$ e $\left.|s|=n\right\}$ então $C=\bigcap_{n \in \omega} C_{n}$ é um conjunto de Cantor.

Demonstração. Considere $\varphi: 2^{\omega} \longrightarrow C$ dada por $\varphi(f)=y$ sendo que $\{y\}=\bigcap_{n \in \omega} F_{f \mid n}$. Afirmação 2.1.18. $\varphi$ está bem definida. 
Demonstração. Fixe $f \in 2^{\omega}$ arbitrária. Observe que $F_{f\lceil n} \subseteq X$ é fechado em $X$ para cada $n \in \omega$ (Definição 2.1.16). Pela Definição 2.1.16 temos que $\operatorname{diam}\left(F_{f \mid n}\right) \stackrel{n \rightarrow \infty}{\longrightarrow} 0$ para cada $n \in \omega$. Como $F_{f \uparrow(n+1)} \subseteq F_{f\lceil n}$ para cada $n \in \omega$ (Definição 2.1.16) e $X$ é um espaço métrico completo então existe um único ponto em $\bigcap_{n \in \omega} F_{f\lceil n} \subseteq X$.

Afirmação 2.1.19. $\varphi$ é injetora.

Demonstração. Considere $f, g \in 2^{\omega}$. Defina $\varphi(f)=y$ e $\varphi(g)=y^{\prime}$. Suponha que $f \neq g$. Então existe $n \in \omega$ tal que $f(n) \neq g(n)$. Seja $n_{0}=\min \{n \in \omega: f(n) \neq g(n)\}$.

Pela Definição 2.1.16 temos $F_{f \uparrow\left(n_{0}+1\right)} \cap F_{g \uparrow\left(n_{0}+1\right)}=\emptyset$. Portanto, $\{y\}=\bigcap_{n \in \omega} F_{f \nmid n} \neq$ $\bigcap_{n \in \omega} F_{g \nmid n}=\left\{y^{\prime}\right\}$. Logo, $\varphi(f) \neq \varphi(g)$.

Afirmação 2.1.20. $\varphi$ é sobrejetora.

Demonstração. Fixe $y \in C$ arbitrário. Então $y \in C_{n}$ para cada $n \in \omega$. Para cada $n \in \omega$ considere $s_{n} \in 2^{<\omega}$ tal que $s_{n}$ possui comprimento igual a $n$ e $y \in F_{s_{n}} \subseteq C_{n}$. Defina $f=\bigcup_{n \in \omega} s_{n}$.

Afirmação 2.1.21. f é uma função de $\omega$ em 2.

Demonstração. Sejam $(a, b),\left(a, b^{\prime}\right) \in f$. Como $f=\bigcup_{n \in \omega} s_{n}$ então existem $m, n \in \omega$ tais que $(a, b) \in s_{n}$ e $\left(a, b^{\prime}\right) \in s_{m}$. Sem perda de generalidade vamos supor que $m \leq n$. Pela Definição 2.1.16 temos que $F_{s_{n}} \subseteq F_{s_{m}}$. Observe que $s_{m} \subseteq s_{n}$ (Definição 2.1.16). Logo, $(a, b),\left(a, b^{\prime}\right) \in s_{n}$. Como $s_{n}$ é uma função então $b=b^{\prime}$.

Observe que $\bigcap_{n \in \omega} F_{f\lceil n}=\bigcap_{n \in \omega} F_{s_{n}}=\{y\}$. Portanto, $\varphi(f)=y$.

Afirmação 2.1.22. $\varphi$ é contínua.

Demonstração. Seja $V \subseteq C$ um aberto básico não vazio de $C$. Fixe $y \in V$ arbitrário. Como $V$ é um aberto básico de $C$ então existem $\varepsilon>0$ e $z \in X$ tais que $V=B_{\varepsilon}(z) \cap C$. Seja $\delta>0$ tal que $B_{\delta}(y) \subseteq B_{\frac{\varepsilon}{2}}(z)$. Note que $B_{\delta}(y) \cap C \subseteq V$.

Como $\varphi$ é uma função sobrejetora então existe $f \in 2^{\omega}$ tal que $\varphi(f)=y$. Pela Definição 2.1.16 temos que $\operatorname{diam}\left(F_{f\lceil n}\right) \stackrel{n \rightarrow \infty}{\longrightarrow} 0$. Então existe $n_{0} \in \omega$ tal que $\operatorname{diam}\left(F_{f\lceil n}\right) \leq$ $\frac{\delta}{2}$ para cada $n \geq n_{0}$. Como $\operatorname{diam}\left(F_{f\lceil n}\right) \leq \frac{\delta}{2}$ para cada $n \geq n_{0}$ então pela definição de $\varphi$ temos que $F_{f\lceil n} \subseteq B_{\delta}(y)$ para cada $n \geq n_{0}$. Como $B_{\delta}(y) \subseteq B_{\varepsilon}(z)$ então $F_{f\lceil n} \subseteq B_{\varepsilon}(z)$ para cada $n \geq n_{0}$. Observe que $\prod_{n \in \omega} U_{n} \subseteq 2^{\omega}$ é aberto básico de $2^{\omega}$ onde: 


$$
U_{n}=\left\{\begin{array}{lr}
\{f(n)\}, & \text { se } n \in\left\{0,1, \ldots, n_{0}\right\} ; \\
2, & \text { caso contrário } .
\end{array}\right.
$$

Suponha que $g \in \prod_{n \in \omega} U_{n}$. Logo, $g(n)=f(n)$ para cada $n \in\left\{0,1, \ldots, n_{0}\right\}$. Então, temos que:

$$
\begin{aligned}
d(z, \varphi(g)) & \leq d(z, \varphi(f))+d(\varphi(f), \varphi(g)) \\
& =d(z, y)+d(y, \varphi(g)) \\
& \leq d(z, y)+\operatorname{diam}\left(F_{f\left\lceil n_{0}\right.}\right) \\
& <\frac{\varepsilon}{2}+\operatorname{diam}\left(F_{f\left\lceil n_{0}\right.}\right) \\
& <\frac{\varepsilon}{2}+\frac{\delta}{2} \\
& <\frac{\varepsilon}{2}+\frac{\varepsilon}{2} \\
& =\varepsilon .
\end{aligned}
$$

Portanto, $\varphi(g) \in B_{\varepsilon}(z) \cap C$. Logo, temos que $\varphi\left[\prod_{n \in \omega} U_{n}\right] \subseteq A$.

Como $2^{\omega}$ é um compacto, $\varphi$ é função bijetora e $X$ é um espaço de Hausdorff (pois $X$ é um espaço métrico) então $\varphi$ é um homeomorfismo.

Abaixo apresentamos uma forma de se construir um conjunto de Cantor que possui medida de Lebesgue igual a zero dentro de um conjunto perfeito não-vazio.

Proposição 2.1.23. Se $X \subseteq \mathbb{R}$ é perfeito e $X \neq \emptyset$ então existe $C \subseteq X$ conjunto de Cantor de medida zero.

Demonstração. Indutivamente podemos construir:

- $X_{\emptyset}=X$.

- Fixe $n<\omega$ arbitrário. Seja $s \in 2^{<\omega}$ tal que $|s|=n$. Suponha construído $X_{s} \subseteq X$ aberto não-vazio em $X$ tal que $\operatorname{diam}\left(X_{s}\right) \leq \frac{1}{2^{n}}$. Considere $x \in X_{s}$. Como $X$ não possui pontos isolados então existe $y \in X_{s}$ tal que $x \neq y$. Sabemos que $X_{s}$ é um espaço normal. Logo, existem $Y_{s{ }_{0}}, Y_{s} \frown_{1} \subseteq X_{s}$ abertos de $X_{s}$ tais 
que $x \in Y_{s \smile_{0}}, y \in Y_{s\urcorner_{1}}$ e $\operatorname{cl}_{\mathbb{R}}\left(Y_{s \smile_{0}}\right) \cap \operatorname{cl}_{\mathbb{R}}\left(Y_{s} \smile_{1}\right)=\emptyset$. Como $X_{s} \subseteq X$ é aberto em $X$ e $Y_{s} \frown_{0}, Y_{s} \frown_{1} \subseteq X_{s}$ são abertos de $X_{s}$ então $Y_{s} \frown_{0}, Y_{s} \smile_{1} \subseteq X_{s}$ abertos de $X$. Defina $\left.X_{s} \frown_{0}=\right] x-\frac{1}{2^{2 n+1}}, x+\frac{1}{2^{2 n+1}}\left[\cap Y_{s} \frown_{0} \mathrm{e} X_{s} \frown_{1}=\right] y-\frac{1}{2^{2 n+1}}, y+\frac{1}{2^{2 n+1}}\left[\cap Y_{s} \frown_{1}\right.$. Observe que $X_{s{ }_{0}}, X_{s} \frown_{1} \subseteq X$ são abertos não-vazios de $X$ tais que:

$$
\begin{aligned}
& \circ x \in X_{s{ }_{0}} \subseteq \mathrm{cl}_{X}\left(X_{s} \smile_{0}\right) \subseteq \mathrm{cl}_{X}\left(Y_{s} \smile_{0}\right) \subseteq \mathrm{cl}_{X}\left(X_{s}\right) ; \\
& \text { ○ } y \in X_{s} \frown_{1} \subseteq \operatorname{cl}_{X}\left(X_{s} \frown_{1}\right) \subseteq \operatorname{cl}_{X}\left(Y_{s} \frown_{1}\right) \subseteq \operatorname{cl}_{X}\left(X_{s}\right) \text {. }
\end{aligned}
$$

Além disso, $\operatorname{diam}\left(X_{s \smile_{0}}\right), \operatorname{diam}\left(X_{s{ }_{1}}\right) \leq \frac{1}{2^{2 n}}$. Como $Y_{s\urcorner_{0}} \cap Y_{s} \frown_{1}=\emptyset$ então $\mathrm{cl}_{X}\left(X_{s} \frown_{0}\right) \cap \mathrm{cl}_{X}\left(X_{s} \smile_{1}\right)=\emptyset$.

- Defina $C_{n}=\bigcup\left\{\operatorname{cl}_{\mathbb{R}}\left(X_{s}\right) \subseteq X: s \in 2^{<\omega}\right.$ e $s$ possui comprimento igual a $\left.n\right\}$ para cada $n \in \omega$. Observe que $C_{n}$ é a reunião de $2^{n}$ fechados de $\mathbb{R}$ para cada $n \in \omega$. Defina $C=\bigcap_{n \in \omega} C_{n}$.

Observe que $\left(\operatorname{cl}_{\mathbb{R}}\left(X_{s}\right)\right)_{s \in 2<\omega}$ é uma estrutura do tipo árvore em $X$ (Definição 2.1.16). Pelo Teorema 2.1.17 temos que $C$ é um conjunto de Cantor.

Resta mostrarmos que $\mu(C)=0$. De fato, $\mu\left(C_{n}\right)=\mu\left(\bigcup_{s \in 2^{n}} \mathrm{cl}_{\mathbb{R}} X_{s}\right) \leq 2^{n} \cdot \frac{1}{2^{2 n}}=\frac{1}{2^{n}}$ para cada $n \in \omega$. Como $\lim _{n \in \omega} \frac{1}{2^{n}}=0$ então temos o resultado.

Abaixo apresentamos a demonstração de que todo fechado não-enumerável da reta real possui um subconjunto de Cantor.

Corolário 2.1.24. Se $X \subseteq \mathbb{R}$ é fechado em $\mathbb{R} e|X|>\aleph_{0}$ então existe um conjunto de Cantor $C \subseteq X$.

Demonstração. Suponha que $X \subseteq \mathbb{R}$ é fechado em $\mathbb{R}$ e $|X|>\aleph_{0}$. Pelo Teorema da Decomposição de Cantor-Bendixson existem $D \subseteq \mathbb{R}$ aberto disperso e $P \subseteq \mathbb{P}$ conjunto perfeito tais que $X=P \cup D$ e $|D| \leq \aleph_{0}$. Observe que $|P|>\aleph_{0}$, pois $|X|>\aleph_{0}$. Assim $P \neq \emptyset$, e o resultado decorre da Proposição 2.1.23.

Introduziremos a seguir uma demonstração para o fato de que é possível construir um conjunto de Cantor que possui medida de Lebesgue igual a zero dentro de todo $G_{\delta}$ denso da reta real.

Teorema 2.1.25. Se $D \subseteq \mathbb{R}$ é um $G_{\delta}$ denso de $\mathbb{R}$ então existe $C \subseteq D$ conjunto de Cantor de medida zero. 
Demonstração. Fixe $U \subseteq \mathbb{R}$ aberto não-vazio em $\mathbb{R}$. Como $D \subseteq \mathbb{R}$ é um $G_{\delta}$ de $\mathbb{R}$ então existe $\left(D_{n}\right)_{n \in \omega}$ tal que $D_{n} \subseteq \mathbb{R}$ é aberto em $\mathbb{R}$ para cada $n \in \omega$ e $D=\bigcap_{n \in \omega} D_{n}$. Como $D \subseteq \mathbb{R}$ é denso em $\mathbb{R}$ e $D \subseteq D_{n}$ para cada $n \in \omega$ então $D_{n} \subseteq \mathbb{R}$ é denso em $\mathbb{R}$ para cada $n \in \omega$.

Indutivamente podemos construir:

- Como $] 0,1\left[\subseteq \mathbb{R}\right.$ é aberto em $\mathbb{R}$ e $D_{0} \subseteq \mathbb{R}$ é um aberto denso de $\mathbb{R}$ então $\left.D_{0} \cap\right] 0,1[\subseteq$ $\mathbb{R}$ é um aberto não vazio de $\mathbb{R}$. Defina $\left.V_{\emptyset}=D_{0} \cap\right] 0,1[$.

- Fixe $n<\omega$ arbitrário. Seja $s \in 2^{<\omega}$ tal que $|s|=n$. Suponha construído $V_{s} \subseteq V_{\emptyset}$ aberto não-vazio em $V_{\emptyset}$. Como $V_{\emptyset}$ é aberto em $\mathbb{R}$ então $V_{s}$ é aberto em $\mathbb{R}$. Fixe $x \in V_{s}$ arbitrário. Considere $\varepsilon>0$ tal que $] x-\varepsilon, x+\varepsilon\left[\subseteq V_{s}\right.$. Tome $y \in$ ]$x-\varepsilon, x+\varepsilon[\operatorname{com} x \neq y$. Escolha $\delta>0$ tal que $[x-\delta, x+\delta] \cap[y-\delta, y+\delta]=\emptyset \mathrm{e}$ $[x-\delta, x+\delta],[y-\delta, y+\delta] \subseteq] x-\varepsilon, x+\varepsilon\left[\right.$. Como $D_{n+1} \subseteq \mathbb{R}$ é um aberto denso em $\mathbb{R}$ então $\left.D_{n+1} \cap\right] x-\delta, x+\delta\left[, D_{n+1} \cap\right] y-\delta, y+\delta[\subseteq \mathbb{R}$ são abertos não-vazios de $\mathbb{R}$. Considere $\delta^{\prime}=\min \left\{\delta, \frac{1}{2^{2 n+1}}\right\}$. Defina $\left.V_{s} \frown_{0}=D_{n+1} \cap\right] x-\delta^{\prime}, x+\delta^{\prime}\left[\subseteq V_{s}\right.$ e $\left.V_{s \frown 1}=D_{n+1} \cap\right] y-\delta^{\prime}, y+\delta^{\prime}\left[\subseteq V_{s}\right.$. Como $V_{s} \frown_{0} \subseteq \mathbb{R}$ é aberto em $\mathbb{R}$ e $V_{s} \frown_{0} \subseteq V_{s}$ então $V_{s} \smile_{0}$ é aberto em $V_{s}$. Como $V_{s} \frown_{1} \subseteq \mathbb{R}$ é aberto em $\mathbb{R}$ e $V_{s \frown_{1}} \subseteq V_{s}$ então $V_{s} \frown_{1}$ é aberto em $V_{s}$.

- Defina $C_{n}=\bigcup\left\{\operatorname{cl}_{\mathbb{R}}\left(V_{s}\right): s \in 2^{<\omega}\right.$ e $s$ possui comprimento igual a $\left.n\right\}$ para cada $n \in \omega$. Observe que $C_{n}$ é a reunião de $2^{n}$ fechados de $\mathbb{R}$ para cada $n \in \omega$. Defina $C=\bigcap_{n \in \omega} C_{n}$.

Observe que $\left(\mathrm{cl}_{\mathbb{R}}\left(V_{s}\right)\right)_{s \in 2^{<\omega}}$ é uma estrutura do tipo árvore em $D$ (Definição 2.1.16). Pelo Teorema 2.1.17 temos que $C$ é um conjunto de Cantor.

Por motivo análogo ao da proposição anterior temos $\mu(C)=0$. 


\subsection{Pequenos Cardinais e $\sigma$-ideais}

Esta seção tem por objetivo apresentar alguns pequenos cardinais e alguns $\sigma$-ideais que são de interesse no decorrer do texto. Adicionalmente, apresentaremos uma caracterização dos conjuntos compactos do espaço de Baire, $\omega^{\omega}$. Analisaremos algumas construções indutivas de famílias de funções no espaço de Baire que nos serão úteis em 3.1.18. Demonstraremos que a cardinalidade da topologia euclidiana da reta real é contínuo, e portanto, a cardinalidade da família dos conjuntos fechados da reta real é contínuo.

Começamos com a definição de pré-ordem, o conceito de anticadeia e a noção da propriedade ccc.

Definição 2.2.1. Dizemos que $\leq$ é uma pré-ordem sobre um conjunto $\mathbb{P}$ se, para cada $a, b, c \in \mathbb{P}$, temos:

(i) $a \leq a$;

(ii) se $a \leq b$ e $b \leq c$ então $a \leq c$.

Definição 2.2.2. Seja $(\mathbb{P}, \leq)$ uma pré-ordem. Dizemos que $p, q \in \mathbb{P}$ são incompatíveis se não existe $r \in \mathbb{P}$ tal que $r \leq p, q$. Notação: $p \perp q$. Dizemos que $p, q \in \mathbb{P}$ são compatíveis se $p, q$ não são incompatíveis. Notação: $p \not \perp q$.

Definição 2.2.3. Seja $(\mathbb{P}, \leq)$ uma pré-ordem. Dizemos que $A \subseteq \mathbb{P}$ é uma anticadeia se, dados $a, b \in A$ distintos, temos que $a \perp b$.

Definição 2.2.4. Seja ( $\mathbb{P}, \leq)$ uma pré-ordem. Dizemos que X satisfaz c.c.c. (countable chain condition) se toda anticadeia em $\mathbb{P}$ é enumerável.

Abaixo apresentamos a pré-ordem usual no espaço de Baire. Adicionalmente, apresentamos uma demonstração de que a relação definida em 2.2.5é, de fato, uma ordem parcial.

Definição 2.2.5. Sejam $f, g \in \omega^{\omega}$. Dizemos que $f \leq^{*} g$ se $\{n \in \omega: f(n)>$ $g(n)\}$ é finito.

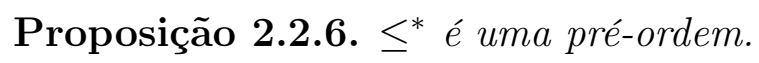


Demonstração. Fixe $f \in \omega^{\omega}$ arbitrária. Note que:

$$
|\{n \in \omega: f(n)>f(n)\}|=|\emptyset|<\aleph_{0} .
$$

Considere $f, g, h \in \omega^{\omega}$ tais que $f \leq^{*} g$ e $g \leq^{*} h$. Observe que:

$$
\{n \in \omega: f(n)>h(n)\} \subseteq\{n \in \omega: f(n)>g(n)\} \cup\{n \in \omega: g(n)>h(n)\} .
$$

Como $|\{n \in \omega: f(n)>g(n)\} \cup\{n \in \omega: g(n)>h(n)\}|<\aleph_{0}$ então $\mid\{n \in \omega: f(n)>$ $h(n)\} \mid<\aleph_{0}$.

A seguir introduziremos o conceito de família dominante e família ilimitada no espaço de Baire.

Definição 2.2.7. Seja $\mathcal{F} \subseteq \omega^{\omega}$. Dizemos que $\mathcal{F}$ é uma familia ilimitada se não existe $g \in \omega^{\omega}$ tal que $f \leq^{*} g$ para cada $f \in \mathcal{F}$. Dizemos que $\mathcal{F}$ é uma família dominante se, para cada $g \in \omega^{\omega}$, existe $f \in \mathcal{F}$ tal que $g \leq^{*} f$.

Exemplo 2.2.8. $\mathcal{F}=\omega^{\omega}$ é uma família dominante e ilimitada.

Abaixo apresentamos a definição de dois pequenos cardinais, $\mathfrak{b}$ e $\mathfrak{d}$.

Definição 2.2.9. Definem-se os seguintes cardinais

$\cdot \mathfrak{b}=\min \left\{|\mathcal{F}|: \mathcal{F} \subseteq \omega^{\omega}\right.$ é uma família ilimitada $\}$

$\cdot \mathfrak{d}=\min \left\{|\mathcal{F}|: \mathcal{F} \subseteq \omega^{\omega}\right.$ é uma família dominante $\}$.

A Proposição 2.2 .10 mostra que $\mathfrak{b} \leq \mathfrak{d}$.

Proposição 2.2.10. Toda familia dominante é ilimitada.

Demonstração. Realizaremos a demonstração da contrapositiva. Seja $\mathcal{F}$ uma família que não é ilimitada. Então, existe $g \in \omega^{\omega}$ tal que $f \leq^{*} g$ para cada $f \in \mathcal{F}$. Considere $h \in \omega^{\omega}$ dada por

$$
h(n)=g(n)+1
$$

para cada $n \in \omega$. 
Afirmação 2.2.11. Não existe $f \in \mathcal{F}$ tal que $h \leq^{*} f$.

Demonstração. Suponha por contradição que existe $f \in \mathcal{F}$ tal que $h \leq^{*} f$. Como $g(n)<h(n)$ para cada $n \in \omega$ temos que $\{n \in \omega: h(n)>g(n)\}=\omega$. Por hipótese, $f \leq^{*} g$, então pela transitividade de $\leq^{*}$ temos $h \leq^{*} g$. Portanto, pela definição de $\leq^{*}$, o conjunto $\{n \in \omega: h(n)>g(n)\}$ é finito, o que é uma contradição, uma vez que $\omega$ é o primeiro ordinal infinito.

Logo, $\mathcal{F}$ não é dominante.

Abaixo apresentamos uma demonstração de que $\aleph_{1} \leq \mathfrak{b}$.

Proposição 2.2.12. Não existe uma família ilimitada enumerável.

Demonstração. Seja $\left(f_{n}\right)_{n \in \omega}$ sequência de funções em $\omega^{\omega}$. Para cada $n \in \omega$, defina

$$
g(n)=\max \left\{f_{0}(n), \ldots, f_{n}(n)\right\} \in \omega .
$$

Note que para cada $n \in \omega$ temos que $\left\{k \in \omega: f_{n}(k)>g(k)\right\} \subseteq\{0, \ldots, n-1\}$ e, consequentemente, $f_{n} \leq^{*} g$ para cada $n \in \omega$. Logo, $\left(f_{n}\right)_{n \in \omega}$ não é ilimitada.

Corolário 2.2.13. $\aleph_{1} \leq \mathfrak{b} \leq \mathfrak{d} \leq \mathfrak{c}$.

Demonstração. Pela Proposição 2.2.12, $\aleph_{1} \leq \mathfrak{b}$. Pelo Exemplo 2.2 .8 temos $\mathfrak{d} \leq \mathfrak{c}$ e $\mathfrak{b} \leq \mathfrak{c}$. Pela Proposição 2.2 .10 temos $\mathfrak{b} \leq \mathfrak{d}$.

O Lema 2.2.14 nos mostra que existe uma partição de $\omega$ em infinitos conjuntos infinitos.

Lema 2.2.14. Existe uma sequência $\left(M_{n}\right)_{n \in \omega}$ tal que $M_{n} \subseteq \omega$ para cada $n \in \omega e$ satisfaz as seguintes propriedades:

(1) $\left|M_{i}\right|=\aleph_{0}$;

(2) $M_{i} \cap M_{j}=\emptyset$ para cada $i, j \in \omega$ tais que $i \neq j$;

(3) $\bigcup_{n \in \omega} M_{n}=\omega$.

(4) $\min \left(M_{n}\right) \geq n$ para cada $n \in \omega$. 
Demonstração. Seja $\left(p_{n}\right)_{n \in \omega}$ uma enumeração crescente dos números primos. Defina $M_{n+1}=\left\{p_{n}^{j}: j \in \omega \backslash\{0\}\right\}$ para $n \in \omega$ e $M_{0}=\omega \backslash \bigcup_{n \in \omega} M_{n+1}$.

Definição 2.2.15. Seja $f \in \omega^{\omega}$. Denotaremos por $C_{f}$ o conjunto $\left\{g \in \omega^{\omega}: g \leq f\right\}$.

No resultado abaixo apresentamos uma caracterização dos conjuntos compactos do espaço de Baire.

Teorema 2.2.16. $X \subseteq \omega^{\omega}$ é compacto se, e somente se, $X$ é fechado em $\omega^{\omega}$ e existe $f \in \omega^{\omega}$ tal que $X \subseteq C_{f}$.

Demonstração. $(\longleftarrow)$ Considere $X_{n}=\{0,1, \ldots, f(n)\}$ para cada $n \in \omega$. Observe que $X_{n} \subseteq \omega$ é compacto em $\omega$ para cada $n \in \omega$. Portanto, pelo Teorema de Tychonoff temos que $C_{f}=\prod_{n \in \omega} X_{n}$ é compacto.

Como $X$ é fechado em $\omega^{\omega}$ e $X \subseteq C_{f}$ então $X$ é fechado em $C_{f}$. Logo, $X$ é compacto. $(\longrightarrow)$ Como $\omega^{\omega}$ é Hausdorff então $X$ é fechado. Considere $\pi_{n}: \omega^{\omega} \longrightarrow \omega$ projeção na $n$-ésima coordenada.

Fixe $n<\omega$. Como $\pi_{n}$ é contínua e $X \subseteq \omega^{\omega}$ é compacto em $\omega^{\omega}$ então $\pi_{n}[X] \subseteq \omega$ é compacto em $\omega$. Logo, $\pi_{n}[X] \in[\omega]^{<\omega}$. Considere $f \in \omega^{\omega}$ dada por $f(n)=\max \pi_{n}[X]+1$ para cada $n \in \omega$. Observe que $X \subseteq C_{f}$.

A Proposição 2.2.17 apresenta uma maneira de relacionarmos os conjuntos compactos do intervalo $[0,1]$ que são disjuntos dos racionais com a caracterização dos compactos do espaço de Baire, apresentada no Teorema 2.2.16.

Proposição 2.2.17. Sejam $\varphi: \mathbb{P} \cap[0,1] \longrightarrow \omega^{\omega}$ um homeomorfismo e $F \subseteq[0,1]$ um fechado em $[0,1]$ tal que $F \cap \mathbb{Q}=\emptyset$. Existe $g \in \omega^{\omega}$ tal que $\varphi[F] \subseteq C_{g}$.

Demonstração. Como $[0,1]$ é um espaço compacto e $F \subseteq[0,1]$ é fechado em $[0,1]$, então $F$ é compacto. Logo, $\varphi[F] \subseteq \omega^{\omega}$ é compacto, pois $\varphi$ é contínua. Portanto, pelo Teorema 2.2.16 existe $g \in \omega^{\omega}$ tal que $\varphi[F] \subseteq C_{g}$.

Apresentamos a seguir uma construção de funções crescentes na pré-ordem $<*$.

Proposição 2.2.18. Se $\mathfrak{b}=\aleph_{1}$ então existe uma família ilimitada $\left\{f_{\alpha}: \alpha<\omega_{1}\right\}$ tal que para cada $\alpha<\beta<\omega_{1}$ temos $f_{\alpha}<^{*} f_{\beta}$.

Demonstração. Seja $\left\{g_{\alpha}: \alpha<\omega_{1}\right\}$ uma família ilimitada em $\omega^{\omega}$. Para obter $\left\{f_{\alpha}: \alpha<\right.$ $\left.\omega_{1}\right\}$ satifazendo as propriedades desejadas iremos proceder por indução transfinita: 
- Defina $f_{0}=g_{0}$;

- Fixe $\eta<\omega_{1}$ arbitrário. Suponha construído $f_{\alpha} \in \omega^{\omega}$ para cada $\alpha<\eta$ tal que $g_{\alpha}<^{*} f_{\alpha}$ para cada $\alpha<\omega_{1}$ e $f_{\alpha}<^{*} f_{\beta}$ para cada $\alpha<\beta<\eta$. Como $\eta<\omega_{1}$ então $\left\{f_{\alpha} \in \omega^{\omega}: \alpha<\eta\right\}$ não é uma família ilimitada em $\omega^{\omega}$. Logo, $\left\{f_{\alpha} \in \omega^{\omega}: \alpha<\eta\right\}$ é limitada em $\omega^{\omega}$. Seja $h_{\eta} \in \omega^{\omega}$ tal que $f_{\alpha}<^{*} h_{\eta}$ para cada $\alpha<\eta$. Considere $f_{\eta} \in \omega^{\omega}$ dada por $f_{\eta}(n)=\max \left\{g_{\eta}(n), h_{\eta}(n)\right\}$ para cada $n \in \omega$.

Considere $\left\{f_{\alpha} \in \omega^{\omega}: \alpha<\omega_{1}\right\}$. Como $g_{\alpha}<^{*} f_{\alpha}$ para cada $\alpha<\omega_{1}$ então a família $\left\{f_{\alpha} \in \omega^{\omega}: \alpha<\omega_{1}\right\}$ é ilimitada em $\omega^{\omega}$. Se $\alpha<\beta<\omega_{1}$ então $f_{\alpha}<^{*} f_{\beta}$ por construção.

A Proposição 2.2.19 exibe uma maneira de relacionar os conjuntos compactos do intervalo $[0,1]$ que são disjuntos dos racionais com famílias crescentes na ordem $<^{*}$.

Proposição 2.2.19. Assuma $\mathfrak{b}=\aleph_{1}$. Seja $\varphi: \mathbb{P} \cap[0,1] \longrightarrow \omega^{\omega}$ um homeomorfismo. Existe $X=\left\{f_{\alpha}: \alpha<\omega_{1}\right\} \subseteq \omega^{\omega}$ tal que para cada $F \subseteq[0,1]$ fechado em $[0,1]$ com $F \cap \mathbb{Q}=\emptyset$ existe $\xi<\omega_{1}$ de maneira que $\varphi[F] \cap X \subseteq\left\{f_{\beta}: \beta \leq \xi\right\}$.

Demonstração. Seja $X=\left\{f_{\alpha}: \alpha<\omega_{1}\right\}$ uma família ilimitada em $\omega^{\omega}$ tal que $f_{\alpha}<^{*} f_{\beta}$ para cada $\alpha<\beta<\omega_{1}$ (Proposição 2.2.18). Considere $g \in \omega^{\omega}$ tal que $\varphi[F] \subseteq C_{g}$ (Proposição 2.2.17). Como $X$ é uma família ilimitada então existe $\xi<\omega_{1}$ tal que $f_{\xi} \mathbb{Z}^{*}$ g. Observe que $f_{\beta} \mathbb{Z}^{*} g$ para cada $\beta>\xi$, pois $f_{\alpha}<^{*} f_{\beta}$ para cada $\alpha<\beta<\omega_{1}$. Logo, temos que $C_{g} \cap X \subseteq\left\{f_{\beta}: \beta \leq \xi\right\}$. Como $\varphi[F] \subseteq C_{g}$ então $\varphi[F] \cap X \subseteq\left\{f_{\beta}: \beta \leq \xi\right\}$.

Exibimos abaixo o conceito de espaço concentrado. Necessitamos dessa noção para determinadas construções, como por exemplo, em 3.1.18.

Definição 2.2.20. Sejam $(X, \tau)$ um espaço topológico e $Y \subseteq X$. Dizemos que $X$ é concentrado em $Y$ se para cada $U \subseteq X$ aberto em $X$ tal que $Y \subseteq U$ temos $X \backslash U$ enumerável.

O Lema 2.2.21 nos mostra uma maneira de construir um conjunto concentrado nos racionais de $[0,1]$ que não é enumerável.

Lema 2.2.21. Assuma $\mathfrak{b}=\aleph_{1}$. Sejam $\varphi: \mathbb{P} \cap[0,1] \longrightarrow \omega^{\omega}$ um homeomorfismo e $X=\left\{f_{\alpha}: \alpha<\omega_{1}\right\} \subseteq \omega^{\omega}$ tal que para cada $F \subseteq[0,1]$ fechado em $[0,1]$ com $F \cap \mathbb{Q}=\emptyset$ existe $\xi<\omega_{1}$ de maneira que $\varphi[F] \cap X \subseteq\left\{f_{\beta}: \beta \leq \xi\right\}$. O conjunto $Y=\varphi^{-1}[X] \cup(\mathbb{Q} \cap[0,1])$ é concentrado em $\mathbb{Q} \cap[0,1]$ e $|Y|>\aleph_{0}$. 
Demonstração. Considere $U \subseteq \mathbb{R}$ aberto em $[0,1]$ tal que $\mathbb{Q} \cap[0,1] \subseteq U$. Logo, $F=$ $[0,1] \backslash U \subseteq[0,1]$ é fechado em $[0,1]$ e, além disso, $F \cap \mathbb{Q}=\emptyset$. Afirmamos que $|Y \backslash U| \leq \aleph_{0}$. Com efeito, como $\mathbb{Q} \cap[0,1] \subseteq U$ então $Y \backslash U \subseteq \varphi^{-1}[X] \backslash U=F \cap \varphi^{-1}[X]$. Por hipótese, $\varphi[F] \cap X \subseteq\left\{f_{\beta}: \beta \leq \xi\right\}$. Como $\xi<\omega_{1}$ então $\left|\left\{f_{\beta}: \beta<\xi\right\}\right| \leq \aleph_{0}$. Logo, temos que $|\varphi[F] \cap X| \leq \aleph_{0}$. Então $\left|F \cap \varphi^{-1}[X]\right| \leq \aleph_{0}$, pois $\varphi$ é um homeomorfismo. Como $Y \backslash U \subseteq F \cap \varphi^{-1}[X]$ e $\left|F \cap \varphi^{-1}[X]\right| \leq \aleph_{0}$ então $|Y \backslash U| \leq \aleph_{0}$. Portanto, $|Y|>\aleph_{0}$.

Abaixo introduzimos o conceito de $\sigma$-ideal.

Definição 2.2.22. Seja $X$ um conjunto. Dizemos $\mathcal{I} \subseteq \wp(X)$ é um $\sigma$-ideal sobre $X$ se $\mathcal{I}$ satisfaz:

(i) $X \notin \mathcal{I} \neq \emptyset$;

(ii) Para cada sequência $\left(A_{n}\right)_{n \in \omega}$ em $\mathcal{I}$ temos que $\bigcup_{n \in \omega} A_{n} \in \mathcal{I}$;

(iii) Se $A \subseteq B \in \mathcal{I}$ então $A \in \mathcal{I}$;

(iv) $\{x\} \in \mathcal{I}$ para cada $x \in X$.

Denotaremos por $\mathcal{N}$ o conjunto $\{X \subseteq \mathbb{R}: X$ tem medida de Lebesgue zero $\}$ e por $\mathcal{M}$ o conjunto $\{X \subseteq \mathbb{R}: X$ é magro $\}$.

Exemplo 2.2.23. $[\mathbb{R}]^{\leq \omega}, \mathcal{N}$ e $\mathcal{M}$ são $\sigma$-ideais sobre $\mathbb{R}$.

Definição 2.2.24. Dado um $\sigma$-ideal $\mathcal{I}$ sobre um conjunto $X$, definem-se os seguintes cardinais:

$$
\begin{aligned}
& \cdot \operatorname{add}(\mathcal{I})=\min \{|\mathcal{A}|: \mathcal{A} \subseteq \mathcal{I} \& \cup \mathcal{A} \notin \mathcal{I}\} \\
& \cdot \operatorname{cov}(\mathcal{I})=\min \{|\mathcal{C}|: \mathcal{C} \subseteq \mathcal{I} \& \cup \mathcal{C}=X\} ; \\
& \cdot \operatorname{non}(\mathcal{I})=\min \{|Y|: Y \subseteq X \& Y \notin \mathcal{I}\} ; \\
& \cdot \operatorname{cof}(\mathcal{I})=\min \{|\mathcal{D}|: \mathcal{D} \subseteq \mathcal{I} \& \forall X \in \mathcal{I} \exists Y \in \mathcal{D}(X \subseteq Y)\}
\end{aligned}
$$

Definição 2.2.25. Sejam $X \neq \emptyset$ e $\mathcal{I}$ um $\sigma$-ideal sobre $X$. Dizemos que $\mathcal{B} \subseteq \mathcal{I}$ é uma base de $\mathcal{I}$ se para cada $I \in \mathcal{I}$ existe $B \in \mathcal{B}$ tal que $I \subseteq B$.

Observação 2.2.26. Sejam $X \neq \emptyset$ e $\mathcal{I}$ um $\sigma$-ideal sobre $X$. Note que $\operatorname{cof}(\mathcal{I})$ é a menor cardinalidade de uma base de $\mathcal{I}$. 
O seguinte fato é um resultado de Teoria da Medida:

Fato 2.2.27. Se $X \subseteq \mathbb{R}$ possui medida de Lebesgue igual a zero então existe $G \subseteq \mathbb{R}$ um $G_{\delta}$ de $\mathbb{R}$ tal que $G$ possui medida de Lebesgue igual a zero e $X \subseteq G$.

Apresentamos abaixo uma demonstração para o fato de que a cardinalidade da família dos subconjuntos da reta real que são $G_{\delta}$ é contínuo.

Proposição 2.2.28. $\mid\left\{G \subseteq \mathbb{R}: G\right.$ é um $\left.G_{\delta} d e \mathbb{R}\right\} \mid=\mathfrak{c}$.

Demonstração. Denote por $\tau$ a topologia usual de $\mathbb{R}$. Sabemos que $|\tau|=\mathfrak{c}$. Note que:

$$
\begin{aligned}
\mid\left\{G \subseteq \mathbb{R}: G \text { é um } G_{\delta} \text { de } \mathbb{R}\right\} \mid & =|\tau|^{\omega} \\
& =\mathfrak{c}^{\omega} \\
& =\left(2^{\omega}\right)^{\omega} \\
& =2^{\omega} \\
& =\mathfrak{c} .
\end{aligned}
$$

De maneira análoga a Proposição 2.2.28, no Corolário 2.2.29 exibimos uma demonstração de que a cardinalidade dos subconjuntos da reta real que são $F_{\sigma}$ é igual a contínuo.

Corolário 2.2.29. $\mid\left\{F \subseteq \mathbb{R}: F\right.$ é um $\left.F_{\sigma} d e \mathbb{R}\right\} \mid=\mathfrak{c}$.

Demonstração. Observe que:

$$
F \text { é um } F_{\sigma} \text { de } \mathbb{R} \longleftrightarrow(\mathbb{R} \backslash F) \text { é um } G_{\delta} \text { de } \mathbb{R} \text {. }
$$

Pela Proposição 2.2.28 temos que:

$$
\begin{aligned}
\mid\left\{F \subseteq \mathbb{R}: F \text { é um } F_{\sigma} \text { de } \mathbb{R}\right\} \mid & =\mid\left\{\mathbb{R} \backslash F: F \text { é um } F_{\sigma} \text { de } \mathbb{R}\right\} \mid \\
& \leq \mid\left\{G \subseteq \mathbb{R}: G \text { é um } G_{\delta} \text { de } \mathbb{R}\right\} \mid \\
& =\mathfrak{c} .
\end{aligned}
$$


Note que:

$$
G \text { é um } G_{\delta} \text { de } \mathbb{R} \longleftrightarrow(\mathbb{R} \backslash G) \text { é um } F_{\sigma} \text { de } \mathbb{R} \text {. }
$$

Pela Proposição 2.2 .28 temos:

$$
\begin{aligned}
\mathfrak{c} & =\mid\left\{G \subseteq \mathbb{R}: G \text { é um } G_{\delta} \text { de } \mathbb{R}\right\} \mid \\
& =\mid\left\{\mathbb{R} \backslash G: G \text { é um } G_{\delta} \text { de } \mathbb{R}\right\} \mid \\
& \leq \mid\left\{F \subseteq \mathbb{R}: F \text { é um } F_{\sigma} \text { de } \mathbb{R}\right\} \mid
\end{aligned}
$$

Exibimos abaixo bases com cardinalidade do contínuo para os ideais de subconjuntos da reta real com medida de Lebesgue igual a zero e subconjuntos magros.

Proposição 2.2.30. Existem $\mathcal{B}, \mathcal{C}$ bases de $\mathcal{N}$ e $\mathcal{M}$ respectivamente tais que $|\mathcal{B}|=$ $|\mathcal{C}|=\mathfrak{c}$.

Demonstração. Defina:

$\mathcal{B}=\left\{G \subseteq \mathbb{R}: G\right.$ é um $G_{\delta}$ de $\mathbb{R}$ e $G$ possui medida de Lebesgue igual a zero $\}$

Pelo Fato 2.2.27 e pela Proposição 2.2.28 temos que $\mathcal{B}$ é uma base de $\mathcal{N}$ tal que $|\mathcal{B}| \leq \mathfrak{c}$

Afirmação 2.2.31. $|\mathcal{B}| \geq \mathfrak{c}$.

Demonstração. Basta notar que $\{x\} \in \mathcal{B}$ para cada $x \in \mathbb{R}$.

Considere:

$$
\mathcal{C}=\left\{F \subseteq \mathbb{R}: F \text { é um } F_{\sigma} \text { de } \mathbb{R}\right\} .
$$

Se $X \subseteq \mathbb{R}$ é magro então existe $F \subseteq \mathbb{R}$ um $F_{\sigma}$ de $\mathbb{R}$ tal que $X \subseteq F$ e, portanto, $\mathcal{C}$ é base de $\mathcal{M}$. Pelo Corolário 2.2 .29 temos que $|\mathcal{C}|=\mathfrak{c}$.

Apresentamos abaixo uma notação para o espaço de funções. 
Notação 2.2.32. Denotamos por $A^{B}$ o conjunto $\{f \subseteq A \times B: f$ é função $\}$.

Exibimos abaixo dois lemas referentes ao cálculo de cardinalidade de conjuntos que nos serão úteis para analisar cardinalidades de conjuntos que não imediatas. Omitimos suas demonstrações por fugirem do escopo deste trabalho.

Lema 2.2.33. Se $\kappa$ é um cardinal infinito $e\left|X_{\alpha}\right| \leq \kappa$ para cada $\alpha<\kappa$, então $\bigcup_{\alpha<\kappa} X_{\alpha} \leq \kappa$.

Lema 2.2.34. Se $\kappa$ um cardinal infinito então $\left|\kappa^{<\omega}\right|=\kappa$.

O Lema 2.2.34 implica que a cardinalidade dos subconjuntos finitos da reta real é contínuo, como apontamos no corolário abaixo.

Corolário 2.2.35. $\left|[\mathbb{R}]^{<\omega}\right|=\mathfrak{c}$.

Exibimos a seguir uma demonstração de que o conjunto dos intervalos da reta real que possuem extremos racionais tem cardinalidade $\aleph_{0}$.

Proposição 2.2.36. Seja $\mathcal{F}$ o conjunto \{]$a, b[: a, b \in \mathbb{Q}\}$. Vale a igualdade $|\mathcal{F}|=\aleph_{0}$.

Demonstração. Note que $] 0, n[\in \mathcal{F}$ para cada $n \in \omega$. Além disso, temos que $] 0, n[\neq] 0, m[$ para cada $m, n \in \omega$ com $m \neq n$. Então $|\{] 0, n[: n \in \omega\}|=\aleph_{0}$. Logo, temos $|\mathcal{F}| \geq \aleph_{0}$.

Considere $f: \mathcal{F} \longrightarrow[\mathbb{Q}]^{<\omega}$ dada por $f(] a, b[)=\{a, b\}$ para cada $] a, b[\in \mathcal{F}$. Afirmo que $f$ é uma função injetora. De fato, considere $] a, b[] c,, d[\in \mathcal{F}$ tais que $] a, b[\neq] c, d[$. Podemos supor sem perda de generalidade que $a \leq b$ e $c \leq d$. Como $] a, b[\neq] c, d[$ então $a \neq c$ ou $b \neq d$. Logo, $\{a, b\} \neq\{c, d\}$. Como $f(] a, b[)=\{a, b\}$ e $f(] c, d[)=\{c, d\}$ então $f(] a, b[) \neq f(] c, d[)$. Portanto, $f$ é injetora.

Logo, temos que:

$$
\begin{aligned}
|\mathcal{F}| & \leq\left|[\mathbb{Q}]^{<\omega}\right| \\
\stackrel{|\mathbb{Q}|=\aleph_{0}}{=} & \left|\left[\aleph_{0}\right]^{<\omega}\right| \\
& =\aleph_{0} .
\end{aligned}
$$

Como $|\mathcal{F}| \leq \aleph_{0}$ e $\aleph_{0} \leq|\mathcal{F}|$ então $|\mathcal{F}|=\aleph_{0}$

No decorrer dessa seção denotaremos por $\tau_{\mathbb{R}}$ o seguinte conjunto:

$$
\{A \subseteq \mathbb{R}: A \text { é aberto em } \mathbb{R}\}
$$


Lema 2.2.37. Existem $\mathfrak{c}$ subconjuntos fechados raros em $\mathbb{R}$.

Demonstração. Sabemos que todo fechado é complementar de um aberto. Existem c abertos em $\mathbb{R}$. Então a cardinalidade de todos os fechados raros em $\mathbb{R}$ é no máximo $\mathfrak{c}$. Sabemos que $\{x\}$ é fechado raro em $\mathbb{R}$ para cada $x \in \mathbb{R}$. Então a cardinalidade de todos os fechados raros em $\mathbb{R}$ é no minímo $\mathfrak{c}$ e, portanto, existem $\mathfrak{c}$ subconjuntos fechados raros em $\mathbb{R}$. 


\subsection{Forcing: alguns resultados e comentários}

O objetivo dessa seção é apresentar alguns resultados e algumas análises pertinentes ao uso de forcing que será realizado na Seção 5.2.

Começaremos com o conceito de imersão densa. Em nosso contexto isso é necessário, pois essas funções nos dão uma noção de equivalência entre forçar com ordens parciais que possuem uma imersão densa entre si.

Definição 2.3.1. Sejam $\left(\mathbb{P}_{1}, \leq_{1}\right)$ e $\left(\mathbb{P}_{2}, \leq_{2}\right)$ ordens parciais. Dizemos que uma função $i: \mathbb{P}_{1} \longrightarrow \mathbb{P}_{2}$ é uma imersão densa se:

(i) para cada $p, q \in \mathbb{P}_{1}$ tais que $p \leq_{1} q$ temos $i(p) \leq_{2} i(q)$;

(ii) para cada $p, q \in \mathbb{P}_{1}$ tais que $p \perp q$ temos $i(p) \perp i(q)$;

(iii) $i\left[\mathbb{P}_{1}\right] \subseteq \mathbb{P}_{2}$ é denso em $\mathbb{P}_{2}$.

Sejam $\mathbb{P}_{0}, \mathbb{P}_{1}$ duas ordens parciais. O próximo resultado mostra que se existe uma imersão densa $\varphi: \mathbb{P}_{0} \longrightarrow \mathbb{P}_{1}$ então $\mathbb{P}_{0}$ e $\mathbb{P}_{1}$ produzem as mesmas extensões genéricas.

Teorema 2.3.2. Sejam $V$ um modelo transitivo enumerável de $Z F C, \mathbb{P}_{0}, \mathbb{P}_{1} \in V$ duas ordens parciais e $\varphi: \mathbb{P}_{0} \longrightarrow \mathbb{P}_{1} \in V$ uma imersão densa.

(1) Se $G_{1} \subseteq \mathbb{P}_{1}$ é um filtro $V$-genérico em $\mathbb{P}_{1}$ então $G_{0} \doteq \varphi^{-1}\left[G_{1}\right]$ é um filtro $V$-genérico em $\mathbb{P}_{0}$. Além disso, $V\left[G_{0}\right]=V\left[G_{1}\right]$;

(2) Se $G_{0} \subseteq \mathbb{P}_{0}$ é um filtro $V$-genérico em $\mathbb{P}_{0}$ então $G_{1} \doteq\left\{q \in \mathbb{P}_{1}: \exists p \in G_{0}(\varphi(p) \leq q)\right\}$ é um filtro $V$-genérico em $\mathbb{P}_{1}$. Além disso, $V\left[G_{0}\right]=V\left[G_{1}\right]$.

O próximo conceito que será introduzido e utilizado na Seção 5.2 é o de ordem separativa. Isso nos interessa, uma vez que ordens separativas e enumeráveis produzem a mesma noção de forcing, como veremos adiante.

Definição 2.3.3. Seja $(\mathbb{P}, \leq)$ uma ordem parcial. Dizemos que $\mathbb{P}$ é separativa se para cada $p \in \mathbb{P}$ existem $r, s \in \mathbb{P}$ tais que $r, s \leq p$ e $r \perp s$.

Iremos introduzir o forcing de Cohen. Na Seção 5.2 iremos introduzir um forcing equivalente a esse. Denotaremos por $F_{u n c}(\omega, 2)$ o conjunto:

$$
\left\{f \subseteq \omega \times 2: f \text { é função, } \operatorname{dom}(f) \subseteq \omega \text { e }|\operatorname{dom}(f)|<\aleph_{0}\right\} .
$$


O próximo exemplo nos mostra que o forcing de Cohen é uma ordem parcial separativa.

Exemplo 2.3.4. Func $_{\omega}(\omega, 2)$ é separativa.

Demonstração. Fixe $p \in \operatorname{Func}_{\omega}(\omega, 2)$ arbitrário. Como $|\operatorname{dom}(p)|<\omega$ então existe $n \in \omega$ tal que $n \notin \operatorname{dom}(p)$. Defina $r=p \cup\{(n, 0)\}$ e $s=p \cup\{(n, 1)\}$. Observe que $r, s \in F \operatorname{Func}_{\omega}(\omega, 2)$ são extensões comuns de $p$. Além disso, $r \perp s$.

O Exemplo 2.3.4 mostra que o forcing de Cohen é uma ordem parcial separativa e a Proposição 2.3.5 mostra que ele também é enumerável. Todo forcing com essas duas propriedades produz as mesmas estensões genéricas, como veremos mais adiante.

Proposição 2.3.5. $\left|F u n c_{\omega}(\omega, 2)\right|=\aleph_{0}$.

Demonstração. Note que $\operatorname{Func}_{\omega}(\omega, 2) \subseteq[\omega \times 2]^{<\omega}$. Logo, temos que:

$$
\begin{aligned}
\left|\operatorname{Func}_{\omega}(\omega, 2)\right| & \leq\left|[\omega \times 2]^{<\omega}\right| \\
& =\left|[\omega]^{<\omega}\right| \\
& =\aleph_{0} .
\end{aligned}
$$

Defina $f_{n}=\{(n, 0)\}$ para cada $n \in \omega$. Note que $f_{n} \in F_{u n c}(\omega, 2)$ para cada $n \in \omega$. Como $f_{n} \neq f_{m}$ para cada $m, n \in \omega$ tais que $m \neq n$ então $\left|\left\{f_{n}: n \in \omega\right\}\right|=\aleph_{0}$. Portanto, $\left|\operatorname{Func}_{\omega}(\omega, 2)\right| \geq \aleph_{0}$.

Seja $\mathbb{P}$ uma ordem parcial não-vazia. Considere $p \in \mathbb{P}$. Denotaremos por $\downarrow p$ o conjunto $\{q \in \mathbb{P}: q \leq p\}$.

O resultado abaixo nos mostra uma maneira de construir indutivamente uma anticadeia maximal enumerável abaixo de um dado elemento em uma ordem parcial separativa.

Lema 2.3.6. Sejam $\mathbb{P}$ uma ordem parcial separativa ccc e $p \in \mathbb{P}$. Existe $A_{p} \subseteq \downarrow p$ anticadeia maximal em $\downarrow p$ tal que $\left|A_{p}\right|=\aleph_{0}$.

Demonstração. Fixe $p \in \mathbb{P}$ arbitrário. Como $\mathbb{P}$ é separativa então existem $p_{0}, q_{0} \in \mathbb{P}$ tais que $p_{0}, q_{0} \leq p$ e $p_{0} \perp q_{0}$. 
Seja $n \in \omega$. Suponha construídos $p_{i}, q_{i}$ para cada $i \in\{0, \ldots, n\}$ em $\downarrow p$ tais que $p_{i+1}, q_{i+1} \leq p_{i}$ para cada $i \in\{0, \ldots, n-1\}$ e $p_{i} \perp q_{i}$ para cada $i \in\{0, \ldots, n\}$. Considere $p_{n} \in \mathbb{P}$. Como $\mathbb{P}$ é separativa então existem $p_{n+1}, q_{n+1} \in \mathbb{P}$ tais que $p_{n+1}, q_{n+1} \leq p_{n} \mathrm{e}$ $p_{n+1} \perp q_{n+1}$. Defina $A=\left\{q_{n}: n \in \omega\right\}$.

Afirmação 2.3.7. A é uma anticadeia em $\mathbb{P}$.

Demonstração. Suponha por absurdo que $A$ não é uma anticadeia em $\mathbb{P}$. Então existem $i, j \in \omega$ com $i \neq j$ tais que $q_{i} \not \not q_{j}$. Sem perda de generalidade suponha que $0 \leq i<j$.

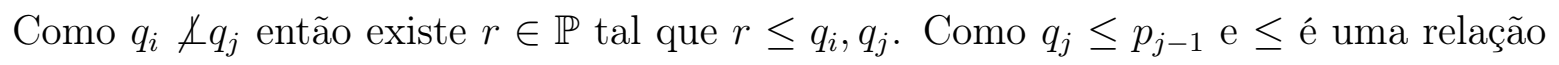
transitiva temos que $r \leq p_{j-1}$. Note que $p_{j-1} \leq p_{i}$. Como $\leq$ é uma relação transitiva e $r \leq p_{j-1}$ então $r \leq p_{i}$. Contradição, pois $p_{i} \perp q_{i}$.

Como $q_{n} \leq p$ para cada $n \in \omega$ então $A \subseteq \downarrow p$.

Defina $\mathcal{A}=\{B \subseteq \downarrow p: B$ é uma anticadeia e $A \subseteq B\}$. Considere $\mathcal{A}$ parcialmente ordenado pela inclusão.

Seja $\left\{B_{i}\right\}_{i \in I}$ uma cadeia em $\mathcal{A}$. Queremos demonstrar que $B=\bigcup_{i \in I} B_{i}$ é um limitante superior de $\left\{B_{i}\right\}_{i \in I}$. Como $B_{i} \subseteq \downarrow p$ para cada $i \in I$ então $B \subseteq \downarrow p$. Como $A \subseteq B_{i}$ para cada $i \in I$ então $A \subseteq B$. Para concluir que $B$ é um majorante superior de $\left\{B_{i}\right\}_{i \in I}$ resta mostrar a seguinte:

Afirmação 2.3.8. B é uma anticadeia em $\mathbb{P}$.

Demonstração. Suponha por absurdo que $B$ não é uma anticadeia em $\mathbb{P}$. Então existem $p, q \in B$ tais que $p \not \perp q$. Como $B=\bigcup_{i \in I} B_{i}$ então existem $i, j \in I$ tais que $p \in B_{i}$ e $q \in B_{j}$. Como $\left\{B_{i}\right\}_{i \in I}$ é uma cadeia em $\mathcal{A}$ então $B_{i} \subseteq B_{j}$ ou $B_{j} \subseteq B_{i}$. Sem perda de generalidade iremos supor que $B_{i} \subseteq B_{j}$. Logo, temos que $p, q \in B_{j}$. Como $B_{j}$ é uma anticadeia em $\mathbb{P}$ então $p \perp q$. Contradição.

Pelo Lema de Kuratowski-Zorn temos que existe $A_{p}$ elemento maximal em $\mathcal{A}$. Como $A \subseteq A_{p}$ e $|A|=\aleph_{0}$ então $\left|A_{p}\right| \geq \aleph_{0}$. Por hipótese, temos que $\mathbb{P}$ é ccc.

Tendo em vista o Teorema 2.3.2, a próxima proposição garante que todas as ordens parciais enumeráveis e separativas produzem as mesmas extensões genéricas.

Proposição 2.3.9. Seja $\mathbb{P}$ uma ordem parcial separativa e enumerável. Considere $\widetilde{\mathbb{P}}=(T, \supset)$, onde $T=\left\{p \in\right.$ Func $\left._{\omega}(\omega, \omega): \operatorname{dom}(p) \in \omega\right\}$. Existe uma imersão densa $\varphi: \widetilde{\mathbb{P}} \longrightarrow \mathbb{P}$. 
Demonstração. Seja $\left\{p_{n}: n \in \omega\right\}$ uma enumeração de $\mathbb{P}$ sem repetições. A ideia é construir $\varphi: \widetilde{\mathbb{P}} \longrightarrow \mathbb{P}$ nível por nível.

Pelo Lema 2.3.6 temos que existe $A_{\emptyset} \subseteq \downarrow p_{0}$ anticadeia maximal em $\mathbb{P}$ tal que $\left|A_{\emptyset}\right|=$ $\aleph_{0}$. Seja $\left\{a_{k}^{\emptyset}: k \in \omega\right\}$ uma enumeração sem repetições de $A_{\emptyset}$. Defina $\varphi(\emptyset)=p_{0} \mathrm{e}$ $\varphi((k))=a_{k}^{\emptyset}$ para cada $k \in \omega$. Observe que as condições $(i)$ e (ii) na Definição 2.3.1 estão satisfeitas.

Fixe $n \in \omega$ arbitrário. Suponha construído $\varphi(p)$ para cada $p \in T$ com $|p| \leq n$ tal que:

(i') $\varphi(p) \leq \varphi(q)$ para cada $p, q \in T$ com $|p|,|q| \leq n$ tais que $p \supseteq q$;

(ii') $\varphi(p) \perp \varphi(q)$ para cada $p, q \in T$ com $|p|,|q| \leq n$ tais que $p \perp q$.

Seja $s \in T$ tal que $|s|=n$. Iremos analisar dois casos:

$p_{n} \perp \varphi(s)$ : Pelo Lema 2.3.6 temos existe $A_{s} \subseteq \downarrow \varphi(s)$ anticadeia maximal em $\mathbb{P}$ tal que $\left|A_{s}\right|=$ $\aleph_{0}$. Seja $\left\{a_{k}^{s}: k \in \omega\right\}$ uma enumeração sem repetições de $A_{s}$. Defina $\varphi\left(s^{\frown}(k)\right)=$ $a_{k}^{s}$ para cada $k \in \omega$.

$p_{n} \not \perp \varphi(s)$ : Como $p_{n} \not \perp \varphi(s)$ então existe $r \in \mathbb{P}$ tal que $r \leq p_{n}, \varphi(s)$. Pelo Lema 2.3 .6 temos que existe $A_{r} \subseteq \downarrow r$ anticadeia maximal em $\mathbb{P}$ tal que $\left|A_{r}\right|=\aleph_{0}$. Como $A_{r} \subseteq \downarrow r$ e $\left|A_{r}\right|=\aleph_{0}$ então existe $s \in A_{r}$ tal que $s \leq r$. Como $r \leq p_{n}, \varphi(s)$ e $\leq$ é uma relação transitiva então $s \leq p_{n}, \varphi(s)$. Seja $\left\{a_{k}^{s}: k \in \omega\right\}$ uma enumeração sem repetições de $A_{s}$. Defina $\varphi(s \frown(k))=a_{k}^{s}$ para cada $k \in \omega$.

Por construção temos que $\varphi$ é uma imersão densa (Definição 2.3.1).

$\mathrm{Na}$ Seção 5.2 iremos introduzir um forcing que em sua essência não é um forcing iterado. Porém iremos enxergá-lo como sendo um forcing iterado, onde todos os forcings são iguais. O Lema do Produto 2.3.10 nos permite quebrar as iterações em etapas para realizarmos análises como as da Observação 2.3.11.

Teorema 2.3.10 (Lema do Produto). Sejam $V$ um modelo transitivo enumerável de $Z F C, \mathbb{P}_{0}, \mathbb{P}_{1} \in V$ duas ordens parciais, $G_{0} \subseteq \mathbb{P}_{0}$ e $G_{1} \subseteq \mathbb{P}_{1}$ filtros em $\mathbb{P}_{0}$ e $\mathbb{P}_{1}$ respectivamente. As seguintes afirmações são equivalentes:

(i) $G_{0} \times G_{1}$ é um filtro $V$-genérico em $\mathbb{P}_{0} \times \mathbb{P}_{1}$;

(ii) $G_{0}$ é um filtro $V$-genérico em $\mathbb{P}_{0}$ e $G_{1}$ é um filtro $V\left[G_{0}\right]$-genérico em $\mathbb{P}_{1}$; 
(iii) $G_{1}$ é um filtro $V$-genérico em $\mathbb{P}_{1}$ e $G_{0}$ é um filtro $V\left[G_{1}\right]$-genérico em $\mathbb{P}_{0}$.

Além disso, se ocorrer alguma das afirmações (i)-(iii) (e, portanto, todas) temos que:

$$
V\left[G_{0} \times G_{1}\right]=V\left[G_{0}\right]\left[G_{1}\right]=V\left[G_{1}\right]\left[G_{0}\right] .
$$

Observação 2.3.11. Seja $Z$ um conjunto. Suponha que $A, B \subseteq Z$ são tais que $A \cap B=$ $\emptyset$ e $Z=A \cup B$. Denotamos por $\mathbb{P}(Z)$ a ordem parcial $\left(F u n c_{\omega}(Z, 2), \supset\right)$. Observe que $\varphi: \mathbb{P}(Z) \longrightarrow \mathbb{P}(A) \times \mathbb{P}(B)$ dada por $\varphi(f)=\left(f_{\lceil A}, f_{\lceil B}\right)$ para cada $f \in F u n c_{\omega}(Z, 2)$ é um isomorfismo de ordem.

Se $G \subseteq \mathbb{P}(Z)$ é um filtro em $\mathbb{P}(Z)$ e $C \subseteq Z$ então definimos $G_{C} \doteq\left\{f_{\lceil C}: f \in G\right\}$. Note que $\varphi[G]=G_{A} \times G_{B}$.

Sejam $V$ um modelo transitivo enumerável de $Z F C, Z, A, B \in V$ tais que $A, B \subseteq Z$, $A \cap B=\emptyset$ e $Z=A \cup B$. Considere $G \subseteq \mathbb{P}(Z)$ um filtro $V$-genérico em $\mathbb{P}(Z)$. Pelo Lema do Produto 2.3.10 temos que $G_{A} \subseteq \mathbb{P}(A)$ é um filtro $V$-genérico em $\mathbb{P}(A), G_{B} \subseteq \mathbb{P}(B)$ é um filtro $V\left[G_{A}\right]$-genérico em $\mathbb{P}(B)$ e:

$$
V[G]=V\left[G_{A} \times G_{B}\right]=V\left[G_{A}\right]\left[G_{B}\right] .
$$

Definição 2.3.12. Fixe $\alpha$ um número ordinal. Seja $\left(\left(Q_{\xi}, \leq_{Q_{\xi}}\right)\right)_{\xi<\alpha}$ uma sequência tal que $\left(Q_{\xi}, \leq_{Q_{\xi}}\right)$ é um forcing para cada $\xi<\alpha$. Para $\beta<\alpha$, defina:

$$
\mathbb{P}_{\beta}=\bigcup\left\{\prod_{\eta \in S} Q_{\eta}: S \in[\beta]^{<\omega}\right\}=\left\{f_{\mid S}: f \in \prod_{\eta<\beta} Q_{\eta} \text { e } S \in[\beta]^{<\omega}\right\} .
$$

A ordem em $\mathbb{P}_{\beta}$ é dada por:

$$
p \leq_{\beta} q \Longleftrightarrow \operatorname{dom}(q) \subseteq \operatorname{dom}(p) \text { e }\left(\forall \eta \in \operatorname{dom}(q)\left(p(\eta) \leq_{Q_{\eta}} q(\eta)\right)\right) .
$$

O lema apresentado abaixo nos diz quando um conjunto entra em uma etapa de iteração de um forcing iterado.

Lema 2.3.13. Sejam $V$ um modelo transitivo enumerável de $Z F C, \kappa \in V$ um número cardinal infinito e regular, $\left(\left(\mathbb{P}_{\xi}, \leq_{\xi}\right)\right)_{\xi \leq \kappa} \in V$ um forcing iterado ccc. Se $Y \in V, G$ é um filtro $V$-genérico em $\mathbb{P}_{\kappa}$ e $S \subseteq Y$ é tal que $V[G] \models|S|<\kappa$; então existe $\zeta<\kappa$ tal que $S \in V\left[G_{\zeta}\right]$, onde $G_{\zeta}=G \cap \mathbb{P}_{\zeta}$. 


\section{Capítulo 3}

\section{Espaços Fortemente Nulos}

\subsection{Definições, Exemplos e Resultados Básicos}

Definição 3.1.1 (Borel [4]). Seja $X \subseteq \mathbb{R}$ um conjunto. Dizemos que $X$ tem medida fortemente nula ou $X$ é fortemente nulo se, para cada sequência de reais positivos $\left(\varepsilon_{n}\right)_{n \in \omega}$, existe uma sequência de intervalos $\left(I_{n}\right)_{n \in \omega}$ tal que $\ell\left(I_{n}\right)<\varepsilon_{n}$ para cada $n \in \omega$ e $X \subseteq \bigcup_{n \in \omega} I_{n}$.

Observe que um argumento por vacuidade demonstra que, o conjunto vazio, $\emptyset$ é fortemente nulo. Note também que, $X \subseteq \mathbb{R}$ é fortemente nulo se, e somente se, para cada sequência de reais positivos $\left(\varepsilon_{n}\right)_{n \in \omega}$, existe uma sequência de intervalos $\left(I_{n}\right)_{n \in \omega}$ tal que $\ell\left(I_{n}\right) \leq \varepsilon_{n}$ para cada $n \in \omega$ e $X \subseteq \bigcup_{n \in \omega} I_{n}$.

Note que, se $X$ fortemente nulo e $Y \subseteq X$ então $Y$ é fortemente nulo. Observe que, todo subconjunto enumerável de $\mathbb{R}$ é fortemente nulo.

Teorema 3.1.2. Seja $X \subseteq \mathbb{R}$. Então $X$ é fortemente nulo se, e somente se, para cada sequência decrescente de reais positivos $\left(\delta_{n}\right)_{n \in \omega}$, existe uma sequência de intervalos com extremos racionais $\left(J_{n}\right)_{n \in \omega}$ tal que $\ell\left(J_{n}\right) \leq \delta_{n}$ para cada $n \in \omega$ e $X \subseteq \bigcup_{n \in \omega} J_{n}$.

Demonstração. Suponha que $X$ é fortemente nulo. Então existe uma sequência de intervalos $\left(I_{n}\right)_{n \in \omega}$ tal que $\ell\left(I_{n}\right)<\delta_{n}$ para cada $n \in \omega$ e $X \subseteq \bigcup_{n \in \omega} I_{n}$.

Fixe $n \in \omega$ arbitrário. Como $\ell\left(I_{n}\right)<\delta_{n}$ então temos que $] 0, \frac{\delta_{n}-\ell\left(I_{n}\right)}{2}[\subseteq \mathbb{R}$ é um intervalo aberto não-vazio de $\mathbb{R}$. Como $\mathbb{Q}$ é denso em $\mathbb{R}$ então existe um número racional $\alpha_{n}$ em $] 0, \frac{\delta_{n}-\ell\left(I_{n}\right)}{2}\left[\right.$. Sejam $a_{n}, b_{n} \in \mathbb{R}$ com $a_{n}<b_{n}$ os extremos do intervalo $I_{n}$. Como 
]$a_{n}-\alpha_{n}, a_{n}[,] b_{n}, b_{n}+\alpha_{n}[\subseteq \mathbb{R}$ são intervalos abertos não-vazios de $\mathbb{R}$ e $\mathbb{Q}$ é denso em $\mathbb{R}$ então existem números racionais $c_{n}, d_{n}$ em $] a_{n}-\alpha_{n}, a_{n}[\mathrm{e}] b_{n}, b_{n}+\alpha_{n}[$ respectivamente. Defina $\left.J_{n}=\right] c_{n}, d_{n}[$. Observe que:

$$
\begin{aligned}
I_{n} & \subseteq\left[a_{n}, b_{n}\right] \\
& \subseteq] c_{n}, d_{n}[ \\
& =J_{n} .
\end{aligned}
$$

Logo, temos que $\bigcup_{n \in \omega} I_{n} \subseteq \bigcup_{n \in \omega} J_{n}$. Portanto, $X \subseteq \bigcup_{n \in \omega} J_{n}$.

Afirmação 3.1.3. Temos que $\ell\left(J_{n}\right)<\delta_{n}$ para cada $n \in \omega$.

Demonstração. Fixe $n \in \omega$ arbitrário. Note que:

$$
\begin{aligned}
\ell\left(J_{n}\right) & \left.<\ell(] a_{n}-\alpha_{n}, b_{n}+\alpha_{n}\right) \\
& =\left(b_{n}+\alpha_{n}\right)-\left(a_{n}-\alpha_{n}\right) \\
& =\left(b_{n}-a_{n}\right)+2 \alpha_{n} \\
& =\ell\left(I_{n}\right)+2 \alpha_{n} .
\end{aligned}
$$

Por construção temos $\alpha_{n}<\frac{\delta_{n}-\ell\left(I_{n}\right)}{2}$. Logo, $\ell\left(I_{n}\right)+2 \alpha_{n}<\delta_{n}$ e, portanto, $\ell\left(J_{n}\right)<$ $\delta_{n}$.

A outra implicação decorre diretamente da Definição 3.1.1. Dada uma sequência de $\left(\varepsilon_{n}\right)_{n \in \omega}$, defina $\delta_{n}=\min \left\{\varepsilon_{k}: k \leq n\right\}$ para cada $n \in \omega$.

Proposição 3.1.4. Seja $X \subseteq \mathbb{R}$ um conjunto fortemente nulo. Então $X$ tem medida de Lebesgue zero.

Demonstração. Seja $\varepsilon>0$ dado. Para cada $n \in \omega$, defina $\varepsilon_{n}=\frac{\varepsilon}{2^{n+1}}$. Como $X$ tem medida fortemente nula, existe uma sequência de intervalos $\left(I_{n}\right)_{n \in \omega}$ tal que $l\left(I_{n}\right) \leq \varepsilon_{n}$ e $X \subseteq \bigcup_{n \in \omega} I_{n}$. Então temos

$$
\sum_{n \in \omega} l\left(I_{n}\right) \leq \sum_{n \in \omega} \varepsilon_{n}=\sum_{n \in \omega} \frac{\varepsilon}{2^{n+1}}=\varepsilon .
$$

Logo, $X$ tem medida de Lebesgue zero. 
É consequência da proposição anterior, que $\mathbb{R}$ não é fortemente nulo.

Denotamos por $\mathcal{S N}$ o conjunto:

$$
\{X \subseteq \mathbb{R}: X \text { tem medida fortemente nula }\} .
$$

Observe que para demonstrarmos que $\mathcal{S N}$ é um $\sigma$-ideal sobre $\mathbb{R}$ basta provarmos a condição (iii) da Definição 2.2.22. Faremos isso no resultado abaixo:

Teorema 3.1.5. $\mathcal{S N}$ é um $\sigma$-ideal sobre $\mathbb{R}$.

Demonstração. Sejam $\left(X_{n}\right)_{n \in \omega}$ uma sequência de conjuntos fortemente nulos e $\left(\varepsilon_{n}\right)_{n \in \omega}$ uma sequência de números reais positivos arbitrária. Considere $\left(P_{n}\right)_{n \in \omega}$ uma partição de $\omega$ em infinitos conjuntos infinitos.

Fixe $m \in \omega$. Como $X_{m}$ é fortemente nulo, existe uma sequência de intervalos $\left(I_{n}\right)_{n \in P_{m}}$ tal que $l\left(I_{n}\right)<\varepsilon_{n}$ para cada $n \in P_{m}$ e $X_{m} \subseteq \bigcup_{n \in P_{m}} I_{n}$. Temos que

$$
\bigcup_{m \in \omega} X_{m} \subseteq \bigcup_{m \in \omega}\left(\bigcup_{n \in P_{m}} I_{n}\right)=\bigcup_{n \in \omega} I_{n} .
$$

Definição 3.1.6 (Borel [4]). Seja $(X, d)$ um espaço métrico. Dizemos que $X$ tem medida fortemente nula ou $X$ é fortemente nulo se, para cada sequência de reais positivos $\left(\varepsilon_{n}\right)_{n \in \omega}$ existe uma sequência $\left(X_{n}\right)_{n \in \omega}$ tal que $\operatorname{diam}\left(X_{n}\right)<\varepsilon_{n}$ para cada $n \in \omega$ e $X=\bigcup_{n \in \omega} X_{n}$.

Seja $(X, d)$ um espaço métrico. Observe que $X$ é fortemente nulo se, e somente se, para cada sequência de reais positivos $\left(\varepsilon_{n}\right)_{n \in \omega}$, existe uma sequência $\left(X_{n}\right)_{n \in \omega}$ tal que $\operatorname{diam}\left(X_{n}\right) \leq \varepsilon_{n}$ para cada $n \in \omega$ e $X \subseteq \bigcup_{n \in \omega} X_{n}$.

Observação 3.1.7. A menos de menção contrária sempre estaremos supondo na Definição 3.1 .6 que $X_{n} \neq \emptyset$ para cada $n \in \omega$.

Proposição 3.1.8. Seja $(X, d)$ um espaço métrico. Então $X$ é fortemente nulo se, e somente se, para cada sequência de números reais positivos $\left(\varepsilon_{n}\right)_{n \in \omega}$ existe uma sequência $\left(x_{n}\right)_{n \in \omega}$ de pontos de $X$ tal que $X=\bigcup_{n \in \omega} B_{\varepsilon_{n}}\left(x_{n}\right)$.

Demonstração. Suponha que $X$ é fortemente nulo. Seja $\left(X_{n}\right)_{n \in \omega}$ tal que $\operatorname{diam}\left(X_{n}\right)<\varepsilon_{n}$ para cada $n \in \omega$ e $X \subseteq \bigcup_{n \in \omega} X_{n}$. Considere $n \in \omega$ arbitrário. Tome $x_{n} \in X_{n}$ qualquer. 
Afirmação 3.1.9. $X_{n} \subseteq B_{\varepsilon_{n}}\left(x_{n}\right)$ para cada $n \in \omega$.

Demonstração. Seja $x \in X_{n}$ arbitrário. Então temos que:

$$
d\left(x, x_{n}\right) \leq \operatorname{diam}\left(X_{n}\right)<\varepsilon_{n}
$$

Logo, $x \in B_{\varepsilon_{n}}\left(x_{n}\right)$.

A Afirmação 3.1 .9 implica $\bigcup_{n \in \omega} X_{n} \subseteq \bigcup_{n \in \omega} B_{\varepsilon_{n}}\left(x_{n}\right)$.

Para outra implicação, fixe uma sequência de números reais positivos $\left(\varepsilon_{n}\right)_{n \in \omega}$ arbitrária. Defina $\delta_{n}=\frac{\varepsilon_{n}}{2}$ para cada $n \in \omega$. Por hipótese, existe uma sequência $\left(x_{n}\right)_{n \in \omega}$ de pontos de $X$ tal que $X=\bigcup_{n \in \omega} B_{\delta_{n}}\left(x_{n}\right)$. Defina $X_{n}=B_{\delta_{n}}\left(x_{n}\right)$. Note que $\operatorname{diam}\left(X_{n}\right)<\left(2 \cdot \delta_{n}\right)=\varepsilon_{n}$ para cada $n \in \omega$.

Definição 3.1.10. A métrica usual de $\omega^{\omega}$ é definida por $\sigma(f, g)=\frac{1}{n+1}$ sendo que $n=\min \{k \in \omega: f(k) \neq g(k)\}$ para cada $f, g \in \omega^{\omega}$ distintas. E a métrica usual de $2^{\omega}$ é a métrica de subespaço induzida no conjunto $2^{\omega}$ por $\sigma$, a qual também denotaremos por $\sigma$.

Observe que a métrica $\sigma$ da Definição 3.1.10 é compatível com a topologia produto.

Teorema 3.1.11. $2^{\omega}$ munido da métrica usual não é fortemente nulo.

Demonstração. De fato, suponha por absurdo que $2^{\omega}$ é um espaço métrico fortemente nulo. Então existe uma sequência $\left(f_{n}\right)_{n \in \omega}$ de funções de $2^{\omega}$ tal que $2^{\omega}=\bigcup_{n \in \omega} B \frac{1}{2 \cdot(n+1)}\left(f_{n}\right)$.

Como $2^{\omega}$ é um espaço compacto então existem $0<n_{0}<n_{1}<\ldots<n_{k}$ tais que $2^{\omega}=\left(\bigcup_{i=0}^{n_{k}} B \frac{1}{2 \cdot\left(n_{i}+1\right)}\left(f_{n_{i}}\right)\right) \cup B_{\frac{1}{2}}\left(f_{0}\right)$. Considere a função $h \in 2^{\omega}$ dada por:

$$
h(n)=\left\{\begin{array}{lr}
f(0)=f_{0}(0)+1 & \text { se } n=0 \\
f_{n_{i}}(n)+1, & \text { se } n=n_{i}-1 \text { para algum } i \in\{0, \ldots, k\} \\
0, & \text { se } n \notin\left\{n_{0}-1, \ldots, n_{k}-1\right\}
\end{array}\right.
$$

Pela Definição 3.1 .10 e pela construção de $h$ temos que $\sigma\left(h, f_{n_{i}}\right) \geq \frac{1}{n_{i}+1}$ para cada $i \in\{0, \ldots, k\}$ e $\sigma\left(h, f_{0}\right) \geq 1$. Então $h \notin B_{\frac{1}{2 \cdot\left(n_{i}+1\right)}}\left(f_{n_{i}}\right)$ para cada $i \in\{0, \ldots, k\}$ e $h \notin B_{\frac{1}{2}}$. Portanto, $h \notin\left(\bigcup_{i=0}^{n_{k}} B \frac{1}{2 \cdot\left(n_{i}+1\right)}\left(f_{n_{i}}\right)\right) \cup B_{\frac{1}{2}}$. Contradição.

Corolário 3.1.12. $\omega^{\omega}$ munido da métrica usual não é fortemente nulo. 
Demonstração. De fato, suponha por absurdo que $\omega^{\omega}$ é fortemente nulo. Então todo subespaço de $\omega^{\omega}$ é fortemente nulo. Em particular, $2^{\omega}$ é fortemente nulo. O que contradiz o Teorema 3.1.11.

A Proposição 3.1 .13 no mostra que a propriedade fortemente nulo é preservada por funções uniformemente contínuas.

Proposição 3.1.13. Sejam $\left(X, d_{X}\right)$ e $\left(Y, d_{Y}\right)$ espaços métricos. Suponha que $X$ é fortemente nulo e $f: X \rightarrow Y$ seja uma função uniformemente contínua. Então $f[X] \subseteq$ $Y$ é fortemente nulo.

Demonstração. Seja $\left(\varepsilon_{n}\right)_{n \in \omega}$ uma sequência de reais positivos. Como $f$ é uniformemente contínua, em particular para cada $\varepsilon_{n}>0$ existe $\delta_{n}>0$ tal que, para todo $A \subseteq X$ com $\operatorname{diam}(A)<\delta_{n}$, temos $\operatorname{diam}(f[A]) \leq \varepsilon_{n}$. Como $X$ é fortemente nulo, existe uma sequência $\left(X_{n}\right)_{n \in \omega}$ tal que $\operatorname{diam}\left(X_{n}\right)<\delta_{n}$ para cada $n \in \omega$ e $X \subseteq \bigcup_{n \in \omega} X_{n}$.

Segue da escolha de $\delta_{n}$ que $\operatorname{diam}\left(f\left[X_{n}\right]\right) \leq \varepsilon_{n}$ para cada $n \in \omega$. Além disso, note que $f[X] \subseteq \bigcup_{n \in \omega} f\left[X_{n}\right]$.

Então, pela definição de conjunto fortemente nulo, segue que $f[X]$ é fortemente nulo.

No que segue iremos demonstrar que a propriedade fortemente nulo não é preservada por funções contínuas. Assumindo a Hipótese do Contínuo, construiremos um subconjunto $Z$ de $[0,1]$ e uma função contínua $\varphi$ de maneira que $\varphi[Z]=2^{\omega}$. Essa construção é devida a Rothberger [14].

Para realizar tal construção precisaremos introduzir o conceito de propriedade de Rothberger, pois o subconjunto $Z$ fortemente nulo será construído de forma indireta utilizando tais conceitos.

Definição 3.1.14. Seja $(X, \tau)$ um espaço topológico. Dizemos que $X$ possui a propriedade de Rothberger se para cada sequência de coberturas abertas $\left(\mathcal{U}_{n}\right)_{n \in \omega}$ de $X$ existe $\left(U_{n}\right)_{n \in \omega}$ tal que $U_{n} \in \mathcal{U}_{n}$ para cada $n \in \omega$ e $X \subseteq \bigcup_{n \in \omega} U_{n}$.

O próximo resultado demonstra que a propriedade de Rothberger implica em fortemente nulo (Miller e Fremlin [3]).

Proposição 3.1.15. Seja $(X, \tau)$ um espaço topológico metrizável. Se X tem a propriedade de Rothberger, então $X$ é fortemente nulo com relação a qualquer métrica que induz a mesma topologia em $X$. 
Demonstração. Suponha que $d$ é uma métrica em $X$ compatível com a sua topologia e que $\left(\varepsilon_{n}\right)_{n \in \omega}$ é uma sequência de reais positivos. Para cada $n \in \omega$, considere o seguinte conjunto:

$$
\mathcal{U}_{n}=\left\{U \subseteq X: U \in \tau \text { e } \operatorname{diam}(U)<\varepsilon_{n}\right\}
$$

Note que $\mathcal{U}_{n}$ é uma cobertura por abertos de $X$ para cada $n \in \omega$. Então, como $X$ tem a propriedade de Rothberger, existe uma cobertura $\left\{U_{n}: n \in \omega\right\}$ tal que $U_{n} \in \mathcal{U}_{n}$ para todo $n \in \omega$. Por construção, temos que $\operatorname{diam}\left(U_{n}\right)<\varepsilon_{n}$ para cada $n \in \omega$ e $X \subseteq \bigcup_{n \in \omega} U_{n}$, pois $\left\{U_{n}: n \in \omega\right\}$ é uma cobertura para $X$. Logo, $X$ é fortemente nulo.

O resultado a seguir nos mostra uma maneira indireta de conseguir espaços com a propriedade de Rothberger.

Proposição 3.1.16. Se $A \subseteq X$ é enumerável e $X$ é concentrado em $A$ então $X$ é Rothberger.

Demonstração. Sejam $\left(\mathcal{U}_{n}\right)_{n \in \omega}$ uma sequência de coberturas abertas para $X$ e $\left\{a_{k}: k \in\right.$ $\omega\}$ uma enumeração para $A$.

Fixe $U_{2 k} \in \mathcal{U}_{2 k}$ tal que $a_{k} \in U_{2 k}$ para cada $k \in \omega$. Defina $V=\bigcup_{k \in \omega} U_{2 k}$. Observe que $V$ é aberto em $X$ e, além disso, $A \subseteq V$. Como $X$ é concentrado em $A$ então $|X \backslash V| \leq \aleph_{0}$. Considere $\left\{b_{k}: k \in \omega\right\}$ uma enumeração para $X \backslash V$. Seja $U_{2 k+1} \in \mathcal{U}_{2 k+1}$ tal que $b_{k} \in U_{2 k+1}$. Logo, $\left\{U_{n}: n \in \omega\right\}$ é cobertura por abertos de $X$.

Abaixo apresentamos uma função que funciona como uma espécie de projeção. Adicionalmetente, demonstramos sua contínuidade em todo domínio. Iremos utilizá-la no Teorema 3.1.18.

Lema 3.1.17. Seja $\pi: \omega^{\omega} \longrightarrow 2^{\omega}$ dada por:

$$
\pi(f)=x \longleftrightarrow \pi(x)(n)=f(n)(\bmod 2) \quad(\forall n \in \omega)
$$

A função $\pi: \omega^{\omega} \longrightarrow 2^{\omega}$ é contínua.

Demonstração. Seja $U \subseteq 2^{\omega}$ um aberto básico de $2^{\omega}$. Sabemos que $U$ é da forma:

$$
\left\{i_{0}\right\} \times \cdots \times\left\{i_{k}\right\} \times 2 \times 2 \times \cdots
$$


onde, $i_{j} \in\{0,1\}$ para cada $j \in\{0, \ldots, k\}$. Pela definição de $\pi$ temos que:

$$
\pi^{-1}[U]=V_{i_{0}} \times \cdots \times V_{i_{k}} \times \omega \times \omega \times \cdots
$$

onde, $V_{i_{j}}=\{2 m: m \in \omega\}$ se $i_{j}=0$ ou $V_{i_{j}}=\{2 m+1: m \in \omega\}$ se $i_{j}=1$ para cada $j \in\{0, \ldots, k\}$. Portanto, $\pi^{-1}[U]$ é um aberto básico de $\omega^{\omega}$.

Teorema 3.1.18 (Rothberger [14]). Assuma $C H$. Existem $Z \subseteq[0,1] \cap \mathbb{P}$ fortemente nulo e $\psi:[0,1] \cap \mathbb{P} \longrightarrow 2^{\omega}$ função contínua tal que $\psi[Z]=2^{\omega}$.

Demonstração. Considere $X$ como em 2.2.19. Seja $\left\{x_{\alpha}: \alpha<\omega_{1}\right\}$ uma indexação sem repetições de $2^{\omega}$.

Indutivamente iremos construir $\left\{g_{\xi}: \xi<\omega_{1}\right\} \subseteq \omega^{\omega}$ que nos auxiliará na definição de $Z$. Defina $g_{0}=f_{0}$. Fixe $\eta<\omega_{1}$ arbitrário. Suponha construido $g_{\xi} \in \omega^{\omega}$ para cada $\xi<\eta$ tal que $\left(g_{\zeta}+1\right) \leq^{*} g_{\xi}$ para cada $\zeta<\xi<\eta$. Como $\eta<\omega_{1}$ então $\left\{g_{\xi}+1: \xi<\eta\right\} \subseteq \omega^{\omega}$ é uma família limitada. Seja $g \in \omega^{\omega}$ tal que $\left(g_{\xi}+1\right) \leq^{*} g$ para cada $\xi<\eta$. Defina $g_{\eta}(n)=\max \left\{g(n), f_{\eta}(n)\right\}$ para cada $n \in \omega$.

Para cada $\alpha<\omega_{1}$ considere $f_{\alpha}^{*} \in \omega^{\omega}$ dada por $f_{\alpha}^{*}(n)=2 \cdot g_{\alpha}(n)+x_{\alpha}(n)$ para cada $n \in \omega$. Defina $X^{*}=\left\{f_{\alpha}^{*}: \alpha<\omega_{1}\right\}$. Considere $\alpha, \beta<\omega_{1}$ tais que $\alpha<\beta$. Observe que:

$$
\begin{aligned}
f_{\alpha}^{*}=2 \cdot g_{\alpha}+x_{\alpha} & \leq 2 \cdot g_{\alpha}+1 \\
& <2 \cdot g_{\alpha}+2 \\
& =2 \cdot\left(g_{\alpha}+1\right) \\
& \leq{ }^{*} 2 \cdot g_{\beta} \\
& \leq 2 \cdot g_{\beta}+x_{\beta}=f_{\beta}^{*} .
\end{aligned}
$$

Afirmação 3.1.19. $X^{*}$ é uma família ilimitada.

Demonstração. Suponha por absurdo que $X^{*}$ não é uma família ilimitada. Então existe $g \in \omega^{\omega}$ tal que $f_{\alpha}^{*} \leq^{*} g$ para cada $\alpha<\omega_{1}$. Observe que $f_{\alpha} \leq^{*} f_{\alpha}^{*}$ para cada $\alpha<\omega_{1}$. Como $\leq^{*}$ é uma ordem parcial sobre $\omega^{\omega}$ então $\leq^{*}$ é uma relação transitiva. Logo, $f_{\alpha} \leq^{*} g$ para cada $\alpha<\omega_{1}$. Contradição, pois $X$ é uma família ilimitada.

Como $\varphi$ é contínua então $\pi \circ \varphi: \mathbb{P} \cap[0,1] \longrightarrow 2^{\omega}$ é contínua. Pelo Lema 2.2.21 temos que $\widetilde{Z}=\varphi^{-1}\left[X^{*}\right] \cup(\mathbb{Q} \cap[0,1])$ é concentrado em $\mathbb{Q} \cap[0,1]$. E, além disso, $Z$ é não- 
enumerável. A Proposição 3.1 .15 e a Proposição 3.1 .16 implicam que $\widetilde{Z}$ é fortemente nulo. Defina $Z=\varphi^{-1}\left[X^{*}\right]$. Note que $Z$ é fortemente nulo.

Afirmamos que $(\pi \circ \varphi)[Z]=2^{\omega}$. De fato, fixe $\alpha<\omega_{1}$ arbitrário. Pela definição da função $\pi$ temos que $\pi\left(f_{\alpha}^{*}\right)=\pi\left(2 \cdot f_{\alpha}+x_{\alpha}\right)=x_{\alpha}$. Logo, $\pi\left[X^{*}\right]=2^{\omega}$. Então temos:

$$
(\pi \circ \varphi)[Z]=\pi[\varphi[Z]]=\pi\left[X^{*}\right]=2^{\omega} .
$$

A seguir exibiremos mais dois exemplos de espaços que não são fortemente nulos.

Corolário 3.1.20. Se $X \subseteq \mathbb{R}$ é um conjunto de Cantor, então $X$ não é fortemente nulo.

Demonstração. Suponha por absurdo que $X$ é fortemente nulo. Como $X \subseteq \mathbb{R}$ é um conjunto de Cantor então existe um homeomorfismo $\varphi: X \longrightarrow 2^{\omega}$. Como $X \subseteq \mathbb{R}$ é compacto então $\varphi: X \longrightarrow 2^{\omega}$ é uma função uniformemente contínua. Então temos que $\varphi[X]=2^{\omega}$ é fortemente nulo, pois $X$ é fortemente nulo e $\varphi: X \longrightarrow 2^{\omega}$ é uma função uniformemente contínua. Contradizendo o Teorema 3.1.11.

Teorema 3.1.21. Não existe um fechado não enumerável $X \subseteq \mathbb{R}$ tal que $X$ é fortemente nulo.

Demonstração. Suponha por absurdo que existe $X \subseteq \mathbb{R}$ tal que $X$ é fechado em $\mathbb{R}$, $|X|>\aleph_{0}$ e $X$ é fortemente nulo. Pelo Corolário 2.1.24 temos que existe um conjunto de Cantor $C \subseteq X$. Como $X$ é fortemente nulo temos que $C$ é fortemente nulo, pois $C \subseteq X$. Contrariando o Corolário 3.1 .20 .

Corolário 3.1.22. Se $P \subseteq \mathbb{R}$ é um conjunto perfeito então $P$ não é fortemente nulo.

Demonstração. Se $P \subseteq \mathbb{R}$ é perfeito então $P \subseteq \mathbb{R}$ é fechado em $\mathbb{R}$. Sabemos que $|P|=\mathfrak{c}$. Aplicando o Teorema 3.1.21 temos o resultado.

Corolário 3.1.23. $\mathbb{P} \subseteq \mathbb{R}$ não é fortemente nulo.

Demonstração. De fato, suponha por absurdo que $\mathbb{P}$ é fortemente nulo. Seja $\psi: \omega^{\omega} \longrightarrow$ $\mathbb{P}$ um homeomorfismo. Denote por $X$ o conjunto $\psi\left[2^{\omega}\right]$.

Afirmação 3.1.24. $X \subseteq \mathbb{P}$ é um conjunto de Cantor. 
Demonstração. Considere $\widetilde{\psi}: 2^{\omega} \longrightarrow X$ dada por $\widetilde{\psi}(f)=\psi(f)$ para cada $f \in 2^{\omega}$, ou seja, $\widetilde{\psi}$ é a restrição de $\psi$ a $2^{\omega}$. Como a restrição de um homemorfismo é um homeomorfismo sobre a imagem, então $\widetilde{\psi}: 2^{\omega} \longrightarrow X$ é um homeomorfismo. Logo, $X \subseteq \mathbb{P}$ é um conjunto de Cantor.

Como $\mathbb{P}$ é fortemente nulo, então todo subspaço de $\mathbb{P}$ é fortemente nulo. Em particular, $X$ é fortemente nulo. O que contraria o Corolário 3.1.20.

Os últimos dois resultados dessa seção são devidos a Kuratowski. Tais resultados demonstram que podemos supor que espaços métricos fortemente nulos são separáveis e zero-dimensionais.

Teorema 3.1.25 (Kuratowski [10]). Suponha que $(X, d)$ é fortemente nulo. Então $X$ é zero-dimensional.

Demonstração. Queremos demonstrar que $X$ é zero-dimensional. Note que basta mostrarmos que para cada $x_{0} \in X$ fixado e para cada $\varepsilon>0$ existe $U \subseteq X$ aberto e fechado tal que $x_{0} \in U$ e $\operatorname{diam}(U)<\varepsilon$.

Fixe $x_{0} \in X$ e seja $\varepsilon>0$ arbitrário. Considere $f: X \rightarrow \mathbb{R}$ dada por $f(x)=d\left(x, x_{0}\right)$, para cada $x \in X$. Pelo Lema 2.1.7, a função $f$ é uniformemente contínua. Portanto, pela Proposição 3.1 .13 temos que $f[X] \subseteq \mathbb{R}$ é fortemente nulo. Em particular, existe $\delta \in] 0, \frac{\varepsilon}{2}$ [ tal que $\delta \notin f[X]$. De fato, suponha por absurdo que não; então para cada $\delta \in] 0, \frac{\varepsilon}{2}[$ temos que $\delta \in f[X]$, o que implica que $] 0, \frac{\varepsilon}{2}[\subseteq f[X]$. O que é uma contradição, pois $f[X]$ é fortemente nulo e, portanto, em particular $f[X]$ tem medida de Lebesgue igual a zero.

Defina o seguinte conjunto:

$$
U=\{x \in X: f(x)<\delta\} .
$$

Afirmamos que $U$ é aberto e fechado e, além disso, $x_{0} \in U$ e $\operatorname{diam}(U)<\varepsilon$.

1. $x_{0} \in U$ : De fato, $f\left(x_{0}\right)=d\left(x_{0}, x_{0}\right)=0<\delta$. Portanto, $x_{0} \in U$.

2. $U$ é aberto: Note que $U=f^{-1}[[0, \delta[]$. Como $f(x) \geq 0$ para cada $x \in X$ temos $f[X] \subseteq[0,+\infty[$. Logo, temos que $[0, \delta[\cap f[X]$ é aberto em $f[X]$ (na topologia de subspaço). Como $f$ é uma função contínua temos que $f^{-1}[[0, \delta[]$ é aberto em $X$. 
3. $U$ é fechado: Note que $U=f^{-1}[[0, \delta]]$, pois $\delta \notin f[X]$. Portanto, $U$ também é fechado, uma vez que $[0, \delta]$ é fechado em $\mathbb{R}$ e $f$ é uma função contínua.

4. $\operatorname{diam}(U)<\varepsilon$ : Suponha por absurdo que não. Então existem $x, x^{\prime} \in U$ tais que $d\left(x, x^{\prime}\right) \geq \varepsilon$. Utilizando a desigualdade triangular temos que

$$
\begin{aligned}
d\left(x, x^{\prime}\right) & \leq d\left(x, x_{0}\right)+d\left(x_{0}, x\right) \\
& =f(x)+f\left(x^{\prime}\right) \\
& <\delta+\delta \\
& =2 \delta \\
& <\varepsilon
\end{aligned}
$$

o que é uma contradição.

Proposição 3.1.26 (Kuratowski [10]). Seja $(X, d)$ um espaço métrico. Se X é fortemente nulo, então $X$ é separável.

Demonstração. Fixe $m \in \omega$. Considere a sequência constante $\left(\varepsilon_{n}^{m}\right)_{n \in \omega}=\left(\frac{1}{2^{m}}\right)_{n \in \omega}$. Como $X$ é fortemente nulo então existe uma sequência $\left(X_{n}^{m}\right)_{n \in \omega}$ de subconjuntos de $X$ tal que $\operatorname{diam}\left(X_{n}^{m}\right)<\frac{1}{2^{m}}$ para cada $n \in \omega$ e $X \subseteq \bigcup_{n \in \omega} X_{n}^{m}$.

Escolha $x_{n}^{m} \in X_{n}^{m}$ para cada $n, m \in \omega$. Defina:

$$
D=\left\{x_{n}^{m}: m, n \in \omega\right\} .
$$

Por construção, $D$ é enumerável. Afirmamos que $D$ é denso em $X$. De fato, sejam $x_{0} \in X$ e $\varepsilon>0$. Devemos mostrar que $B_{\varepsilon}\left(x_{0}\right) \cap D \neq \emptyset$. Como $\frac{1}{2^{m}} \stackrel{m \rightarrow \infty}{\longrightarrow} 0$ então existe $m_{0} \in \omega$ tal que $\frac{1}{2^{m_{0}}}<\varepsilon$. Considere a sequência constante $\left(\frac{1}{2^{m_{0}}}\right)_{n \in \omega}$. Como $X$ é fortemente nulo então existe uma sequência $\left(X_{n}^{m_{0}}\right)_{n \in \omega}$ de subconjuntos de $X$ tal que $\operatorname{diam}\left(X_{n}^{m_{0}}\right)<\frac{1}{2^{m_{0}}}$ para cada $n \in \omega$ e $X \subseteq \bigcup_{n \in \omega} X_{n}^{m_{0}}$. Em particular, existe $n_{0} \in \omega$ tal que $x_{0} \in X_{n_{0}}^{m_{0}}$.

Como $\operatorname{diam}\left(X_{n_{0}}^{m_{0}}\right)<\varepsilon$ então $X_{n_{0}}^{m_{0}} \subseteq B_{\varepsilon}\left(x_{0}\right)$. De fato, se $y \in X_{n_{0}}^{m_{0}}$ então temos: 


$$
d\left(x_{0}, y\right) \leq \operatorname{diam}\left(X_{n_{0}}^{m_{0}}\right)<\frac{1}{2^{m_{0}}}<\varepsilon .
$$

Logo, $y \in B_{\varepsilon}\left(x_{0}\right)$. Então, temos que $x_{n_{0}}^{m_{0}} \in B_{\varepsilon}\left(x_{0}\right) \cap D$. 


\section{$3.2 \quad$ Aditividade de $\mathcal{S N}$}

Na seção anterior demonstramos que $\mathcal{S N}$ é um $\sigma$-ideal. Em particular, $\mathcal{S N}$ é fechado para uniões de comprimento $\aleph_{0}$. Nessa seção iremos demonstrar que o Axioma de Martin implica que $\mathcal{S N}$ é fechado pra uniões de comprimento estritamente menor que c.

Definição 3.2.1. Sejam $X$ um conjunto, $\mathcal{I} \subseteq \wp(X)$ um ideal sobre $X$ e $\kappa$ um número cardinal. Dizemos que $\mathcal{I}$ é $\kappa$-completo se para cada $\mathcal{A} \subseteq \mathcal{I}$ com $|\mathcal{A}|<\kappa$ temos que $\bigcup \mathcal{A} \in \mathcal{I}$.

Definição 3.2.2. Seja $\left(x_{k}\right)_{k \in \omega}$ uma sequência de naturais estritamente crescente. Dizemos que $\left(x_{k}\right)_{k \in \omega}$ tem densidade zero se $\lim _{n \rightarrow+\infty} \frac{b_{n}}{n}=0$, onde $b_{n}=\left|\left\{k \in \omega: x_{k} \leq n\right\}\right|$.

Observação 3.2.3. Note que $|\{k \in \omega: k+1 \leq c\}|=\lfloor c\rfloor \leq c$ para cada número real c.

Lema 3.2.4. Para cada $\left(a_{n}\right)_{n \in \omega}$ sequência não decrescente de números naturais com $a_{n} \leq n$ para cada $n \in \omega$ e $\lim _{n \rightarrow+\infty} \frac{a_{n}}{n}=0$ temos que existe $\left(y_{k}\right)_{k \in \omega}$ estritamente crescente de densidade zero tal que $a_{n}+\left|\left\{k \in \omega: y_{k} \leq n\right\}\right| \leq n$ para cada $n \in \omega$.

Demonstração. Defina $y_{0}=0$. Fixe $k \geq 1$ arbitrário e suponha que $y_{j}$ foi definido para cada $j<k$. Iremos demonstrar que vale a desigualdade $\left|\left\{m \in \omega: m-a_{m}<k+1\right\}\right|<\aleph_{0}$.

De fato, defina $M_{k}=\left\{m \in \omega: m-a_{m}<k+1\right\}$. Suponha por absurdo que $\left|M_{k}\right|=\aleph_{0}$. Seja $\left\{m_{i}: i \in \omega\right\}$ uma enumeração sem repetições de $M \backslash\{0\}$ tal que $m_{i}<m_{i+1}$ para cada $i \in \omega$. Então para cada $i \in \omega$ temos que:

$$
\frac{m_{i}-(k+1)}{m_{i}}<\frac{a_{m_{i}}}{m_{i}}
$$

Tomando o limite em ambos os lados na desigualdade acima temos:

$$
\begin{aligned}
\lim _{i \in \omega} \frac{m_{i}-(k+1)}{m_{i}} & =\lim _{i \in \omega} 1-\frac{k+1}{m_{i}} \\
& =1-\lim _{i \in \omega} \frac{k+1}{m_{i}} \\
& =1-0 \\
& =1
\end{aligned}
$$




$$
\begin{aligned}
& <\lim _{i \in \omega} \frac{a_{m_{i}}}{m_{i}} \\
& =0 .
\end{aligned}
$$

Portanto, $1<0$. Contradição.

Denote por $A_{k}$ o conjunto $\left\{m \in \omega: m>y_{k-1}, \sqrt{m} \geq k+1, m-a_{m} \geq k+1\right\}$. Considere $k_{0}=\max \left\{y_{k-1},(k+1)^{2}, \max \left(M_{k}\right)\right\}$. Observe que $\left\{n \in \omega: n>k_{0}\right\} \subseteq A_{k}$. Logo, temos que $A_{k}$ é cofinito em $\omega$. Seja $y_{k}=\max \left(\omega \backslash A_{k}\right)+1$. Por construção, temos que $\left(y_{k}\right)_{k \in \omega}$ é estritamente crescente. Note que $|\{k \in \omega: k+1 \leq \sqrt{n}\}| \leq \sqrt{n}$ para cada $n \in \omega$ (Observação 3.2.3).

Iremos provar que $a_{n}+\left|\left\{k \in \omega: y_{k} \leq n\right\}\right| \leq n$ para cada $n \in \omega$. Seja $k \in \omega$ tal que $y_{k} \leq n$. Então $n \in A_{k}$. Logo, $n-a_{n} \geq k+1$. Então temos $\left\{k \in \omega: y_{k} \leq n\right\} \subseteq\{k \in$ $\left.\omega: n-a_{n} \geq k+1\right\}$.

Portanto, $\left|\left\{k \in \omega: y_{k} \leq n\right\}\right| \leq\left|\left\{k \in \omega: n-a_{n} \geq k+1\right\}\right| \leq n-a_{n}$. Por fim, temos $a_{n}+\left|\left\{k \in \omega: y_{k} \leq n\right\}\right| \leq a_{n}+\left(n-a_{n}\right)=n$.

Iremos demonstrar que $\left(y_{k}\right)_{k \in \omega}$ possui densidade zero. Por construção temos $y_{k} \geq$ $(k+1)^{2}$. Logo, temos que:

$$
\begin{aligned}
\left\{k \in \omega: y_{k} \leq n\right\} & \subseteq\left\{k \in \omega:(k+1)^{2} \leq n\right\} \\
& =\{k \in \omega: k+1 \leq \sqrt{n}\} .
\end{aligned}
$$

Note que $\left|\left\{k \in \omega: y_{k} \leq n\right\}\right| \leq|\{k \in \omega: k+1 \leq \sqrt{n}\}|=\lfloor\sqrt{n}\rfloor \leq \sqrt{n}$. Portanto, $\lim _{n \in \omega} \frac{\left|\left\{k \in \omega: y_{k} \leq n\right\}\right|}{n}=\lim _{n \in \omega} \frac{\sqrt{n}}{n}=0$.

Definição 3.2.5. Seja $\bar{\varepsilon}=\left(\varepsilon_{n}\right)_{n \in \omega}$ uma sequência decrescente de reais positivos. Defina o seguinte conjunto:

$$
\mathbb{P}(\bar{\varepsilon})=\left\{p \in[\mathcal{F}]^{\aleph_{0}}: \forall n \in \omega\left|\left\{I \in p: l(I)>\varepsilon_{n}\right\}\right| \leq n \text { e } \frac{1}{n}\left|\left\{I \in p: l(I)>\varepsilon_{n}\right\}\right| \stackrel{n \rightarrow \infty}{\longrightarrow} 0\right\} .
$$

Considere a seguinte ordem sobre $\mathbb{P}(\bar{\varepsilon}): p \leq q$ se $p \supseteq q$.

Lema 3.2.6. Seja $\bar{\varepsilon}=\left(\varepsilon_{n}\right)_{n \in \omega}$ uma sequência decrescente de reais positivos. Fixe $p \in \mathbb{P}(\bar{\varepsilon})$. Se $\left\{I_{n}: n \in \omega\right\}$ é uma enumeração sem repetições de $p$ tal que $\ell\left(I_{n+1}\right) \leq \ell\left(I_{n}\right)$ para cada $n \in \omega$ então $\ell\left(I_{n}\right) \leq \varepsilon_{n}$ para cada $n \in \omega$.

Demonstração. Suponha por absurdo que existe $n_{0} \in \omega$ tal que $\ell\left(I_{n_{0}}\right)>\varepsilon_{n_{0}}$. Como 
$\ell\left(I_{n+1}\right) \leq \ell\left(I_{n}\right)$ para cada $n \in \omega$ então $\ell\left(I_{n}\right) \geq \varepsilon_{n_{0}}$ para cada $n \in\left\{0,1, \ldots, n_{0}\right\}$. Portanto, temos $\left|\left\{I \in p: \ell(I)>\varepsilon_{n_{0}}\right\}\right| \geq n_{0}+1>n_{0}$. Contradição, pois $p \in \mathbb{P}(\bar{\varepsilon})$.

Lema 3.2.7. Se $\bar{\varepsilon}=\left(\varepsilon_{n}\right)_{n \in \omega}$ é uma sequência decrescente de números reais positivos então $\mathbb{P}(\bar{\varepsilon})$ é ccc.

Demonstração. Suponha por absurdo que existe $\mathcal{A} \subseteq \mathbb{P}(\bar{\varepsilon})$ uma anticadeia em $\mathbb{P}(\bar{\varepsilon})$ não enumerável. Note que podemos supor que $|\mathcal{A}|=\aleph_{1}$. Seja $\left\{p_{\xi}: \xi<\omega_{1}\right\}$ uma indexação sem repetições de $\mathcal{A}$.

Fixe $\xi<\omega_{1}$. Como $p_{\xi} \in \mathbb{P}(\bar{\varepsilon})$ pela Definição 3.2.5 existe $n_{\xi} \in \omega$ tal que para cada $n \geq n_{\xi}$ temos $\frac{1}{n}\left|\left\{I \in p_{\xi}: l(I)>\varepsilon_{n}\right\}\right|<\frac{1}{2}$. Defina $p_{\xi}^{\prime}=\left\{I \in p_{\xi}: l(I)>\varepsilon_{n_{\xi}}\right\} \in[\omega]^{<\aleph_{0}}$. Como existe apenas uma quantidade enumerável de escolhas para $n_{\xi}$ e para $p_{\xi}^{\prime}$, existem $\xi, \eta<\omega_{1}$ distintos tais que $n_{\xi}=n_{\eta}$ e $p_{\xi}^{\prime}=p_{\eta}^{\prime}$.

Afirmamos que $p_{\xi} \cup p_{\eta}$ é uma condição em $\mathbb{P}(\bar{\varepsilon})$. De fato, pela Definição 3.2.5 temos que $p_{\xi}$ e $p_{\eta}$ são conjuntos enumeráveis. Como a reunião de dois conjuntos enumeráveis é um conjunto enumerável temos, $p_{\xi} \cup p_{\eta}$ enumerável. Se $I \in p_{\xi} \cup p_{\eta}$ então $I \in p_{\xi}$ ou $I \in p_{\eta}$. Sem perda de generalidade suponha que $I \in p_{\xi}$. Pela Definição 3.2.5 segue que $I \in \mathcal{F}$. Logo, $p_{\xi} \cup p_{\eta} \in[\mathcal{F}]^{\aleph_{0}}$.

Note que $\left\{I \in p_{\xi} \cup p_{\eta}: l(I)>\varepsilon_{n}\right\} \subseteq\left\{I \in p_{\xi}: l(I)>\varepsilon_{n}\right\} \cup\left\{I \in p_{\eta}: l(I)>\varepsilon_{n}\right\}$ para cada $n \in \omega$. Então, para cada $n \in \omega$ temos:

$$
\frac{1}{n}\left|\left\{I \in p_{\xi} \cup p_{\eta}: l(I)>\varepsilon_{n}\right\}\right| \leq \frac{1}{n}\left|\left\{I \in p_{\xi}: l(I)>\varepsilon_{n}\right\}\right|+\frac{1}{n}\left|\left\{I \in p_{\eta}: l(I)>\varepsilon_{n}\right\}\right| .
$$

Como $p_{\xi}, p_{\eta} \in \mathbb{P}(\bar{\varepsilon})$ pela Definição 3.2 .5 , temos $\frac{1}{n}\left|\left\{I \in p_{\xi}: l(I)>\varepsilon_{n}\right\}\right| \stackrel{n \rightarrow \infty}{\longrightarrow} 0 \mathrm{e}$ $\frac{1}{n}\left|\left\{I \in p_{\eta}: l(I)>\varepsilon_{n}\right\}\right| \stackrel{n \rightarrow \infty}{\longrightarrow} 0$. Portanto, $\frac{1}{n}\left|\left\{I \in p_{\xi} \cup p_{\eta}: l(I)>\varepsilon_{n}\right\}\right| \stackrel{n \rightarrow \infty}{\longrightarrow} 0$.

Fixe $n \in \omega$. Note que $\left|\left\{I \in p_{\xi} \cup p_{\eta}: l(I)>\varepsilon_{n}\right\}\right| \leq\left|\left\{I \in p_{\xi}: l(I)>\varepsilon_{n}\right\}\right|+\mid\left\{I \in p_{\eta}:\right.$ $\left.l(I)>\varepsilon_{n}\right\} \mid \leq \frac{n}{2}+\frac{n}{2}=n$, pois $\frac{1}{n}\left|\left\{I \in p_{\xi}: l(I)>\varepsilon_{n}\right\}\right|<\frac{1}{2}, n_{\xi}=n_{\eta}$ e $p_{\xi}^{\prime}=p_{\eta}^{\prime}$.

Observe que $p_{\xi} \cup p_{\eta} \supseteq p_{\xi}, p_{\eta}$. Contradição, pois $\mathcal{A}$ é uma anticadeia em $\mathbb{P}(\bar{\varepsilon})$.

Definição 3.2.8. Considere $\bar{\varepsilon}=\left(\varepsilon_{n}\right)_{n \in \omega}$ uma sequência decrescente de números reais positivos. Sejam $\kappa$ um número cardinal infinito com $\kappa<\mathfrak{c}$ e $\left(X_{\alpha}\right)_{\alpha<\kappa}$ uma sequência tal que $X_{\alpha} \subseteq \mathbb{R}$ é fortemente nulo para cada $\alpha<\kappa$. Definimos $D_{\alpha}$ como sendo o conjunto $\left\{p \in \mathbb{P}(\bar{\varepsilon}): X_{\alpha} \subseteq \bigcup p\right\}$ para cada $\alpha<\kappa$. 
Lema 3.2.9. Fixe $\bar{\varepsilon}=\left(\varepsilon_{n}\right)_{n \in \omega}$ uma sequência decrescente de números reais positivos. Temos que $D_{\alpha} \subseteq \mathbb{P}(\bar{\varepsilon})$ é denso em $\mathbb{P}(\bar{\varepsilon})$ para cada $\alpha<\kappa$.

Demonstração. Fixe $\alpha<\kappa$ arbitrário. Sejam $p \in \mathbb{P}(\bar{\varepsilon})$ e $\left(n_{i}\right)_{i \in \omega}$ sequência estritamente crescente de naturais satisfazendo:

(i) $\frac{1}{n}\left|\left\{i \in \omega: n_{i} \leq n\right\}\right| \stackrel{n \rightarrow \infty}{\longrightarrow} 0$;

(ii) $\left|\left\{I \in p: l(I)>\varepsilon_{n}\right\}\right|+\left|\left\{i \in \omega: n_{i} \leq n\right\}\right| \leq n$.

Note que $\left(n_{i}\right)_{i \in \omega}$ existe pelo Lema 3.2.4. Como $X_{\alpha} \subseteq \mathbb{R}$ é um conjunto fortemente nulo então temos que existe uma sequência $\left(I_{i}\right)_{i \in \omega} \subseteq[\mathcal{F}]^{\aleph_{0}}$ tal que $l\left(I_{i}\right)<\varepsilon_{n_{i}}$ para cada $i \in \omega$ e $X_{\alpha}=\bigcup_{i \in \omega} I_{i}$ (Teorema 3.1.2.

Defina $p^{\prime}=\left\{I_{i}: i \in \omega\right\}$. Iremos demonstrar que $q=p \cup p^{\prime}$ é uma condição em $\mathbb{P}(\bar{\varepsilon})$. De fato, como $p \in \mathbb{P}(\bar{\varepsilon})$ segue da Definição 3.2 .5 que $p$ é enumeravel. Note que $p^{\prime}$ também é enumerável. Como a reunião de dois conjuntos enumeráveis é um conjunto enumerável temos que $p \cup p^{\prime}$ é um conjunto enumerável. Se $I \in p \cup p^{\prime}$ então $I \in p$ ou $I \in p^{\prime}$. Se $I \in p$ então pela Definição 3.2 .5 temos que $I \in \mathcal{F}$. Se $I \in p^{\prime}$ então $I=I_{j}$ para algum $j \in \omega$. Como $I_{i} \in \mathcal{F}$ para cada $i \in \omega$ então, em particular, $I_{j} \in \mathcal{F}$. Logo, $p \cup p^{\prime} \in[\mathcal{F}]^{\aleph_{0}}$.

Note que $\left\{I \in q: l(I)>\varepsilon_{n}\right\} \subseteq\left\{I \in p: l(I)>\varepsilon_{n}\right\} \cup\left\{I \in p^{\prime}: l(I)>\varepsilon_{n}\right\}$. Então, para cada $n \in \omega$ temos:

$$
\frac{1}{n}\left|\left\{I \in q: l(I)>\varepsilon_{n}\right\}\right| \leq \frac{1}{n}\left|\left\{I \in p: l(I)>\varepsilon_{n}\right\}\right|+\frac{1}{n}\left|\left\{I \in p^{\prime}: l(I)>\varepsilon_{n}\right\}\right| .
$$

Como $p \in \mathbb{P}(\bar{\varepsilon})$ pela Definição 3.2.5, temos $\frac{1}{n}\left|\left\{I \in p: l(I)>\varepsilon_{n}\right\}\right| \stackrel{n \rightarrow \infty}{\longrightarrow} 0$. Segue da condição $(i)$ que $\frac{1}{n}\left|\left\{I \in p^{\prime}: l(I)>\varepsilon_{n}\right\}\right| \stackrel{n \rightarrow \infty}{\longrightarrow} 0$. Logo, $\frac{1}{n}\left|\left\{I \in q: l(I)>\varepsilon_{n}\right\}\right| \stackrel{n \rightarrow \infty}{\longrightarrow} 0$.

Decorre da maneira como foram indexados os intervalos que compõem $p^{\prime}$ e do fato de $p \in \mathbb{P}_{c}$ que $\left|\left\{I \in q: l(I)>\varepsilon_{n}\right\}\right| \leq n$ para cada $n \in \omega$.

Observe que $X_{\alpha}=\bigcup_{n \in \omega} I_{n} \subseteq \bigcup q$. Então pela Definição 3.2 .8 temos $q \in D_{\alpha}$. Como $q$ estende $p$ e $p$ foi escolhido de maneira arbitrária então $D_{\alpha} \subseteq \mathbb{P}(\bar{\varepsilon})$ é denso em $\mathbb{P}(\bar{\varepsilon})$.

Teorema 3.2.10 $\left(M A_{\kappa}\right)$. Seja $\kappa$ um número cardinal infinito tal que $\kappa<\mathfrak{c}$. Se $X_{\alpha} \in$ $\mathcal{S N}$ para cada $\alpha<\kappa$ então $X=\bigcup_{\alpha<\kappa} X_{\alpha} \in \mathcal{S N}$. 
Demonstração. Fixe $\bar{\varepsilon}=\left(\varepsilon_{n}\right)_{n \in \omega}$ uma sequência decrescente de reais positivos. Pelo Lema 3.2 .9 temos que $D_{\alpha} \subseteq \mathbb{P}(\bar{\varepsilon})$ é denso em $\mathbb{P}(\varepsilon)$ para cada $\alpha<\kappa$. Como estamos assumindo a afirmação $M A_{\kappa}$ então existe $G \subseteq \mathbb{P}(\bar{\varepsilon})$ um filtro tal que $G \cap D_{\alpha} \neq \emptyset$ para cada $\alpha<\kappa$. Defina $p^{\star}=\bigcup G$.

Iremos demonstrar que vale a desigualdade $\left|\left\{J \in p^{\star}: \ell(J)>\varepsilon_{n}\right\}\right| \leq n$ para cada $n \in \omega$. De fato, fixe $n \in \omega$ arbitrário. Suponha por absurdo que $\mid\left\{J \in p^{\star}: \ell(J)>\right.$ $\left.\varepsilon_{n}\right\} \mid>n$. Então temos que $\left|\left\{J \in p^{\star}: \ell(J)>\varepsilon_{n}\right\}\right| \geq n+1$. Sejam $J_{0}, J_{1}, \ldots, J_{n} \in p^{\star}$ distintos tais que $\ell\left(J_{i}\right)>\varepsilon$ para cada $i \in\{0,1, \ldots, n\}$. Como $p^{\star}=\bigcup G$ então existem $p_{0}, p_{1}, \ldots, p_{n} \in G$ tais que $J_{i} \in p_{i}$ para cada $i \in\{0,1, \ldots, n\}$. Como $G$ é um filtro então existe $q \in G$ tal que $q \supseteq p_{i}$ para cada $i \in\{0,1, \ldots, n\}$. Logo, temos que $J_{i} \in q$ para cada $i \in\{0,1, \ldots, n\}$. Como $G \subseteq \mathbb{P}(\bar{\varepsilon})$ então $q \in \mathbb{P}(\bar{\varepsilon})$. Pela Definição 3.2 .5 temos que $\left|\left\{I \in q: l(I)>\varepsilon_{n}\right\}\right| \leq n$. Contradição.

Seja $\left\{J_{n}: n \in \omega\right\}$ uma enumeração sem repetições de $p^{*}$ tal que $\ell\left(J_{n+1}\right) \leq \ell\left(J_{n}\right)$ para cada $n \in \omega$. Note que $\ell\left(J_{n}\right) \leq \varepsilon_{n}$ para cada $n \in \omega$ (Lema 3.2.6).

Fixe $\alpha<\kappa$. Como $G \cap D_{\alpha} \neq \emptyset$ existe $p \in G$ tal que $X_{\alpha} \subseteq \bigcup p$ e, portanto, $X=\bigcup_{\alpha<\kappa} X_{\alpha} \subseteq \bigcup(\bigcup G)$. Logo, $X \in \mathcal{S N}$.

Corolário 3.2.11. $M A_{\kappa} \rightarrow \mathcal{S N}$ é $\kappa$-completo.

Corolário 3.2.12 (Carlson [5]). Assuma MA. Seja $\kappa$ um número cardinal infinito tal que $\kappa<$ c. Se $X_{\alpha} \in \mathcal{S N}$ para cada $\alpha<\kappa$ então $\bigcup_{\alpha<\kappa} X_{\alpha} \in \mathcal{S N}$. 


\subsection{Translações}

Nessa seção apresentaremos uma caracterização para conjuntos fortemente nulos de $\mathbb{R}$ que é devida a Galvin, Mycielski e Solovay [6], mas a demonstração que realizaremos aqui é uma modificação da prova original. Tal modificação é devida a Miller [2].

Definição 3.3.1. Sejam $X, Y \subseteq \mathbb{R}$. Definimos $X+Y$ como sendo o conjunto:

$$
\{x+y: x \in X \text { e } y \in Y\} .
$$

Observação 3.3.2. A função reflexão $r: \mathbb{R} \longrightarrow \mathbb{R}$ dada por $r(x)=-x$ para cada $x \in \mathbb{R}$ é uma isometria. Portanto, um homeomorfismo. Então temos que:

1. se $A \subseteq \mathbb{R}$ é raro então $-A$ é raro

2. se $M \in \mathcal{M}$ então $-M \in \mathcal{M}$

3. $s e-X \in \mathcal{S N}$ então $X \in \mathcal{S N}$

4. se existe $x \in \mathbb{R}$ tal que $X+x \in \mathcal{S N}$ então $X \in \mathcal{S N}$.

Lema 3.3.3. Sejam $X, A \subseteq \mathbb{R}$. Se existe $x \in \mathbb{R}$ tal que $(X+x) \cap(-A)=\emptyset$, então $X+A \neq \mathbb{R}$. Vale a recíproca.

Demonstração. $(\longrightarrow)$ Observe que para demonstrarmos que $X+A \neq \mathbb{R}$ basta provar que $-x \in \mathbb{R} \backslash(X+A)$. Afirmamos que $-x \in \mathbb{R} \backslash(X+A)$. De fato, suponha por absurdo que existem $x^{\prime} \in X$ e $a \in A$ tais que $-x=x^{\prime}+a$. Note que $-a=x^{\prime}+x$. Como $x^{\prime}+x \in X+x$ então $-a \in X+x$. Como $-a \in-A$ então $-a \in(X+x) \cap(-A)$. Contradição.

$(\longleftarrow)$ Por hipótese, $X+A \neq \mathbb{R}$. Então existe $x \in \mathbb{R} \backslash(X+A)$. Afirmo que $X \cap(-A+x)=\emptyset$. De fato, suponha por absurdo que existe $y \in X \cap(-A+x)$. Então existe $n \in-A$ tal que $y=n+x$ e $y \in X$. Logo, $x=y-n$. Como $-n \in A$ então $x \in X+A$. Contradição.

Lema 3.3.4. Seja $X \subseteq \mathbb{R}$. Se para cada $M \subseteq \mathbb{R}$ conjunto magro temos que $X+M \neq \mathbb{R}$, então $X$ é fortemente nulo. 
Demonstração. Fixe $\left(\varepsilon_{n}\right)_{n \in \omega}$ uma sequência de números reais positivos arbitrária. Considere $\left\{q_{n}: n \in \omega\right\}$ uma enumeração sem repetições de $\mathbb{Q}$. Note que $\left.D=\bigcup_{n \in \omega}\right] q_{n}-\frac{\varepsilon_{n}}{2}, q_{n}+\frac{\varepsilon_{n}}{2}[$ é um aberto denso de $\mathbb{R}$. Logo, $M=\mathbb{R} \backslash D$ é um fechado raro de $\mathbb{R}$.

Defina $N=M \cup(-M)$. A Observação 3.3 .2 implica que $N \in \mathcal{M}$. Por hipótese, $X+N \neq \mathbb{R}$. Então existe $x \in \mathbb{R} \backslash(X+N)$. Pelo Lema3.3.3 tems que $(X+x) \cap(-N)=\emptyset$. Logo, $N \cap(-X-x)=\emptyset$. Como $M \subseteq N$ então $(-X-x) \cap M=\emptyset$. Portanto, temos que:

$$
\left.-X-x \subseteq(\mathbb{R} \backslash M)=D=\bigcup_{n \in \omega}\right] q_{n}-\frac{\varepsilon_{n}}{2}, q_{n}+\frac{\varepsilon_{n}}{2}[.
$$

Como $\ell(] q_{n}-\frac{\varepsilon_{n}}{2}, q_{n}+\frac{\varepsilon_{n}}{2}[)=\varepsilon_{n}$ para cada $n \in \omega$ então $-X-x$ é fortemente nulo. Como $-X-x=X+(-x)$ então pela Observação 3.3 .2 temos que $X$ é fortemente nulo.

O resultado a seguir nos fornece uma das implicações do Teorema de Galvin, Mycielski e Solovay.

Teorema 3.3.5. Seja $X \subseteq \mathbb{R}$. Se para cada $M \subseteq \mathbb{R}$ magro temos que existe $x \in \mathbb{R}$ tal que $(X+x) \cap M=\emptyset$, então $X$ é fortemente nulo.

Demonstração. Fixe $M \subseteq \mathbb{R}$ conjunto magro arbitrário. Por hipótese, temos que existe $x \in \mathbb{R}$ tal que $(X+x) \cap M=\emptyset$. Pelo Lema 3.3 .3 temos que $X+M \neq \mathbb{R}$. Pelo Lema 3.3 .4 temos que $X$ é fortemente nulo.

Inicialmente começaremos demonstrando a outra implicação em $\mathbb{K}$ e usaremos a função $\varphi: \mathbb{R} \longrightarrow \mathbb{K}$ dada por $\varphi(x)=x-\lfloor x\rfloor$ para cada $x \in \mathbb{R}$ para consiguir $o$ resultado desejado em $\mathbb{R}$. O motivo é que $\mathbb{K}$ é um espaço métrico compacto e podemos usar o Lema da Cobertura de Lebesgue enunciado e provado a seguir.

Teorema 3.3.6 (Lema da Cobertura de Lebesgue). Suponha que $(X, d)$ é um espaço métrico compacto e $\mathcal{C}$ é uma cobertura por abertos de $X$. Então existe $\delta>0$ tal que para cada $Y \subseteq X$ com $\operatorname{diam}(Y) \leq \delta$ existe $C \in \mathcal{C}$ tal que $Y \subseteq C$.

Demonstração. Seja $\mathcal{U}=\left\{U_{i}\right\}_{i \in I}$ uma cobertura por abertos de $X$. Como $X$ é compacto então existe $\left\{U_{0}, \ldots, U_{n}\right\} \subseteq \mathcal{U}$ subcobertura finita de $X$. Defina $F_{i}=X \backslash U_{i}$ para cada $i \in\{0, \ldots, n\}$. Seja $f: X \longrightarrow \mathbb{R}$ dada por $f(x)=\frac{1}{n} \sum_{i=0}^{n} d\left(x, F_{i}\right)$ para cada $x \in X$. Observe que $f$ é uma função uniformente contínua. Em particular, $f$ é contínua. 
Note que $f(x) \geq 0$ para cada $x \in X$. Iremos provar que $f(x)>0$ para cada $x \in X$. De fato, suponha por absurdo que $f(x)=0$ para algum $x \in X$. Então $d\left(x, F_{i}\right)=0$ para cada $i \in\{0, \ldots, n\}$. Logo, $x \in F_{i}$ para cada $i \in\{0, \ldots, n\}$ e, portanto, $x \notin U_{i}$ para cada $i \in\{0, \ldots, n\}$. Contradição, pois $\bigcup_{i=0}^{n} U_{i}=X$.

Como $X$ é compacto e $f: X \longrightarrow \mathbb{R}$ é uma função contínua então $f[X] \subseteq \mathbb{R}$ é compacto. Seja $\delta=\min \{f(x): x \in X\}$. Como $f(x)>0$ para cada $x \in X$ então $\delta>0$. Observe que $f(x) \geq \delta$ para cada $x \in X$ (definição de minímo de um conjunto).

Sejam $Y \subseteq X$ tal que $\operatorname{diam}(Y) \leq \delta$ e $y \in Y$. Existe $i \in\{0, \ldots, n\}$ tal que $d\left(y, F_{i}\right) \geq \delta$. De fato, suponha por absurdo que $d\left(y, F_{i}\right)<\delta$ para cada $i \in\{0, \ldots, n\}$. Então temos que:

$$
\begin{aligned}
f(y) & =\frac{1}{n} \sum_{i=0}^{n} d\left(y, F_{i}\right) ; \\
& <\frac{1}{n} \sum_{i=0}^{n} \delta ; \\
& =\frac{1}{n}(n \cdot \delta) ; \\
& =\delta .
\end{aligned}
$$

Contradizendo a minimalidade de $\delta$.

Iremos demonstrar que $Y \subseteq B_{\delta}[y] \subseteq U_{i}$. Seja $y^{\prime} \in Y$. Como $\operatorname{diam}(Y) \leq \delta$ e $y \in Y$ então $d\left(y^{\prime}, y\right) \leq \operatorname{diam}(Y) \leq \delta$. Portanto, $Y \subseteq B_{\delta}[y]$. Como $d\left(y, F_{i}\right) \geq \delta$ então $y \in U_{i}$. Suponha por absurdo que $B_{\delta}[y] \nsubseteq U_{i}$. Então existe $y^{\prime} \in B_{\delta}[y]$ tal que $y^{\prime} \notin U_{i}$. Como $y^{\prime} \in B_{\delta}[y]$ então $d\left(y^{\prime}, y\right) \leq \delta$. Por outro lado $d\left(y^{\prime}, y\right)>\delta$, pois $y^{\prime} \notin U_{i}$. Contradição.

Como mencionamos, demonstraremos a recíproca do Teorema de Galvin, Mycielski e Solovay inicialmente em $\mathbb{K}$. Para isso, utilizaremos uma noção equivalente de conjuntos fortemente nulos de $\mathbb{K}$ que é dada pela proposição abaixo.

Proposição 3.3.7. Um conjunto $X \subseteq \mathbb{K}$ é fortemente nulo se e somente se para cada sequência de números reais positivos $\left(\varepsilon_{n}\right)_{n \in \omega}$ existe uma sequência de intervalos $\left(I_{n}\right)_{n \in \omega}$ de $\mathbb{K}$ tal que $\ell\left(I_{n}\right) \leq \varepsilon_{n}$ para cada $n \in \omega$ e $X \subseteq \bigcap_{n \in \omega} \bigcup_{m \geq n} I_{m}$.

Demonstração. $(\longrightarrow)$ Fixe $\left(\varepsilon_{n}\right)_{n \in \omega}$ uma sequência de números reais positivos arbitrária. Considere $\left(M_{n}\right)_{n \in \omega}$ uma partição de $\omega$.. Como $X \subseteq \mathbb{K}$ é um conjunto fortemente 
nulo então para cada $n \in \omega$ existe uma sequência de intervalos $\left(I_{k}\right)_{k \in M_{n}}$ de $\mathbb{K}$ tal que $\ell\left(I_{k}\right) \leq \varepsilon_{k}$ para cada $k \in M_{n}$ e $X \subseteq \bigcup_{k \in M_{n}} I_{k}$.

Afirmamos que vale a inclusão $X \subseteq \bigcap_{n \in \omega} \bigcup_{m \geq n} I_{m}$. De fato, sejam $x \in X$ e $n \in \omega$ arbitrários. Como $X \subseteq \bigcup_{k \in M_{n}} I_{k}$ então existe $k \in M_{n}$ tal que $x \in I_{k}$. Pela condição (2) do Lema 2.2 .14 temos que $M_{i} \cap M_{j}=\emptyset$ para cada $i, j \in \omega$ tais que $i \neq j$. Logo, $\left|\left\{k \in \omega: x \in I_{k}\right\}\right|=\aleph_{0}$. Então existe $m_{0} \in\left\{k \in \omega: x \in I_{k}\right\}$ tal que $m_{0} \geq n$. Portanto, $x \in I_{m_{0}} \subseteq \bigcup_{m \geq n} I_{m}$.

$(\longleftarrow)$ Fixe uma sequência de números reais positivos $\left(\varepsilon_{n}\right)_{n \in \omega}$ arbitrária. Existe uma sequência de intervalos $\left(I_{n}\right)_{n \in \omega}$ de $\mathbb{K}$ tal que $\ell\left(I_{n}\right) \leq \varepsilon_{n}$ para cada $n \in \omega$ e $X \subseteq \bigcap_{m \in \omega} \bigcup_{n \geq m} I_{n}$. Fixe $x \in X$ arbitrário. Note que $x \in \bigcup_{n \geq 0} I_{n}=\bigcup_{n \in \omega} I_{n}$.

O Lema a seguir nos fornece uma maneira de conseguirmos uma cobertura para $\mathbb{K}$ com uma propriedade adicional que posteriormente será utilizada na construção de uma árvore.

Lema 3.3.8. Sejam $C \subseteq \mathbb{K}$ fechado raro e $J \subseteq \mathbb{K}$ intervalo fechado não degenerado. Para cada $x \in \mathbb{K}$ existem $I_{x} \subseteq \mathbb{K}$ intervalo aberto e $J_{x} \subseteq J$ intervalo fechado não degenerado tais que $x \in I_{x}$ e $\left(I_{x}+J_{x}\right) \cap C=\emptyset$.

Demonstração. Fixe $x \in \mathbb{K}$ arbitrário. Seja $y$ o ponto médio do intervalo $J$. Iremos analisar dois casos:

$\underline{x+y \in C}:$ Começaremos esse caso analisando que para cada $\delta \in] 0,1[$ existe $s \in] 0, \delta[$ tal que $x+(y+s) \in \mathbb{K} \backslash C$. De fato, suponha por absurdo que existe $\left.\delta_{0} \in\right] 0,1[$ tal que para cada $s \in] 0, \delta_{0}[$ temos que $x+(y+s) \in C$. Então temos $] 0, \delta_{0}[+(x+y) \subseteq C$. Como $] 0, \delta_{0}[+(x+y)=] x+y, \delta_{0}+(x+y)[($ Lema 2.1.3 $)$ e $] 0, \delta_{0}[+(x+y) \subseteq C$ então ]$x+y, \delta_{0}+(x+y)\left[\subseteq C\right.$. Logo, temos $\operatorname{int}_{\mathbb{K}}(] x+y, \delta_{0}+(x+y)[) \subseteq \operatorname{int}_{\mathbb{K}}(C)=\emptyset$. Contradição, pois $] x+y, \delta_{0}+(x+y)[\neq \emptyset$.

Sejam $a, b \in \mathbb{K}$ tais que $J=[a, b]$. Defina $\delta=y-a$. Como $C \subseteq \mathbb{K}$ é fechado em $\mathbb{K}$ então existe $r>0$ tal que (]$(x+(y+s))-r,(x+(y+s))+r[) \cap C=\emptyset$. Pelo Lema 2.1 .2 temos $](x+(y+s))-r,(x+(y+s))+r[=] x-\frac{r}{2}, x+\frac{r}{2}[+$ ]$(y+s)-\frac{r}{2},(y+s)+\frac{r}{2}[$. Logo, $] x-\frac{r}{2}, x+\frac{r}{2}[+](y+s)-\frac{r}{2},(y+s)+\frac{r}{2}[\cap C=$ $\emptyset$. Defina $\left.I_{x}=\right] x-\frac{r}{2}, x+\frac{r}{2}\left[\right.$. Note que $J_{0} \doteq\left[(y+s)-\frac{r}{2},(y+s)+\frac{r}{2}\right] \cap J \subseteq \mathbb{K}$ é um intervalo fechado de $\mathbb{K}$. Seja $r_{0}>0$ tal que $r_{0}<\frac{r}{2}$. Note que $\left[y-r_{0}, y+r_{0}\right] \subseteq$ $J_{0}$. Defina $J_{x}=\left[y-r_{0}, y+r_{0}\right] \cap J$. Como $J_{x} \subseteq J_{0}$ e $J_{0} \subseteq J$ então $J_{x} \subseteq J_{0}$. 
$\underline{x+y \in(\mathbb{K} \backslash C)}:$ Como $C \subseteq \mathbb{K}$ é fechado em $\mathbb{K}$ então existe $r \in \mathbb{K}$ tal que $](x+y)-r,(x+y)+r[\cap C=$ $\emptyset$. Pelo Lema 2.1.2 temos $](x+y)-r,(x+y)+r[=] x-\frac{r}{2}, x+\frac{r}{2}[+] y-\frac{r}{2}, y+\frac{r}{2}[$. Logo, $] x-\frac{r}{2}, x+\frac{r}{2}[+] y-\frac{r}{2}, r+\frac{r}{2}\left[\cap C=\emptyset\right.$. Defina $\left.I_{x}=\right] x-\frac{r}{2}, x+\frac{r}{2}[$. Note que $J_{0} \doteq\left[y-\frac{r}{2}, y+\frac{r}{2}\right] \cap J \subseteq \mathbb{K}$ é um intervalo fechado de $\mathbb{K}$. Seja $r_{0}>0$ tal que $r_{0}<\frac{r}{2}$. Note que $\left[y-r_{0}, y+r_{0}\right] \subseteq J_{0}$. Defina $J_{x}=\left[y-r_{0}, y+r_{0}\right] \cap J$. Como $J_{x} \subseteq J_{0}$ e $J_{0} \subseteq J$ então $J_{x} \subseteq J_{0}$.

Lema 3.3.9. Sejam $C \subseteq \mathbb{K}$ um fechado raro e $J \subseteq \mathbb{K}$ um intervalo fechado não degenerado. Então existem uma família finita e não vazia $\mathcal{A}$ de subintervalos fechados não degenerados de $J$ e $\varepsilon>0$ tais que para cada intervalo $I \subseteq \mathbb{K}$ com $\ell(I)<\varepsilon$ temos que existe $\tilde{J} \in \mathcal{A}$ satisfazendo $(I+\tilde{J}) \cap C=\emptyset$.

Demonstração. Pelo Lema 3.3 .8 temos que para cada $x \in \mathbb{K}$ existem $I_{x} \subseteq \mathbb{K}$ intervalo aberto e $J_{x} \subseteq J$ intervalo fechado não degenerado tais que $x \in I_{x}$ e $\left(I_{x}+J_{x}\right) \cap C=\emptyset$. Observe que $\left\{I_{x}: x \in \mathbb{K}\right\}$ é uma cobertura por abertos de $\mathbb{K}$. Como $\mathbb{K}$ é compacto então existem $n \in \omega$ e $x_{0}, x_{1}, \ldots, x_{n} \in \mathbb{K}$ tais que $\mathbb{K}=\bigcup_{i=0}^{n} I_{x_{i}}$. Considere $\varepsilon>0$ dado pelo Lema da Cobertura de Lebesgue. Defina $\mathcal{A}=\left\{J_{x_{i}}: i \in\{0,1, \ldots, n\}\right\}$.

Seja $I \subseteq \mathbb{K}$ um intervalo tal que $\ell(I)<\varepsilon$. Observe que existe $i \in\{0,1, \ldots, n\}$ tal que $I \subseteq I_{x_{i}}$. Logo, $I+J_{x_{i}} \subseteq I_{x_{i}}+J_{x_{i}}$. Por construção temos que $\left(I_{x_{i}}+J_{x_{i}}\right) \cap C=\emptyset$. Portanto, $\left(I+J_{x_{i}}\right) \cap C=\emptyset$.

Teorema 3.3.10. Se $X \subseteq \mathbb{K}$ é um conjunto fortemente nulo então para cada $F \subseteq \mathbb{K}$ conjunto magro temos que existe $x \in \mathbb{K}$ tal que $(X+x) \cap F=\emptyset$.

Demonstração. Fixe $F \subseteq \mathbb{K}$ um conjunto magro em $\mathbb{K}$ arbitrário. Como $F \subseteq \mathbb{K}$ é um conjunto magro então existe uma sequência de fechados raros $\left(C_{n}\right)_{n \in \omega}$ tal que $C_{n} \subseteq C_{n+1}$ para cada $n \in \omega$ e $F \subseteq \bigcup_{n \in \omega} C_{n}$.

Iremos construir $\left(\varepsilon_{s}\right)_{s \in \omega<\omega}$ e $\left(J_{s}\right)_{s \in \omega<\omega}$ satisfazendo as propriedades:

1. $J_{t}$ é um subintervalo fechado não degenerado ou vazio de $J_{s}$ para cada $s, t \in \omega^{<\omega}$ $\operatorname{com} t \subseteq s$

2. se $I \subseteq \mathbb{K}$ é um intervalo com $\ell(I)<\varepsilon_{s}$ então existe $k \in \omega$ tal que $\left(I+J_{s}{ }^{(k)}\right) \cap$ $C_{|s|}=\emptyset$ para cada $s \in \omega^{<\omega}$. 
Pelo Lema 3.3 .8 existem uma família finita e não vazia $\mathcal{A}_{\emptyset}$ de subintervalos fechados não degenerados de $\mathbb{K}$ e $\varepsilon_{\emptyset}>0$ tais que para cada intervalo $I \subseteq \mathbb{K}$ com $\ell(I)<\varepsilon_{\emptyset}$ temos que existe $J_{\emptyset} \in \mathcal{A}_{\emptyset}$ satisfazendo $\left(I+J_{\emptyset}\right) \cap C_{0}=\emptyset$.

Considere $n \in \omega$ arbitrário. Suponha construídos números reais positivos $\varepsilon_{s}$ para cada $s \in \omega^{<\omega} \operatorname{com}|s|<n$ e intervalos fechados $J_{s} \subseteq \mathbb{K}$ para cada $s \in \omega^{<\omega}$ com $|s| \leq n$ tais que:

(i) $J_{t}$ é um subintervalo fechado não degenerado ou vazio de $J_{s}$ para cada $s, t \in \omega^{<\omega}$ tais que $|s|,|t| \leq n$ e $t \subseteq s$;

(ii) se $I \subseteq \mathbb{K}$ é um intervalo com $\ell(I)<\varepsilon_{s}$ então existe $k \in \omega$ tal que $\left(I+J_{s}{ }_{(k)}\right) \cap$ $C_{|s|}=\emptyset$ para cada $s \in \omega^{<\omega}$ tal que $|s|<n$.

Seja $s \in{ }^{n} \omega$ arbitrário. Considere $C_{n}$ e $J_{s}$. Iremos analisar dois casos:

$\underline{J_{s}=\emptyset}:$ Defina $J_{s} \overbrace{(m)}=\emptyset$ para cada $m \in \omega$ e $\varepsilon_{s}=0$.

$\underline{J_{s} \neq \emptyset}$ : Pelo Lema 3.3 .9 existem uma família finita $\mathcal{A}_{s}$ de subintervalos fechados não degenerados de $J_{s}$ e $\varepsilon_{s}>0$ tais que para cada $I \subseteq \mathbb{K}$ com $\ell(I)<\varepsilon_{s}$ temos que existe $J \in \mathcal{A}_{s}$ tal que $(I+J) \cap C_{|s|}=\emptyset$. Defina $\left|\mathcal{A}_{s}\right|=k_{s}$. Seja $\left\{J_{s}^{0}, J_{s}^{1}, \ldots, J_{s}^{k_{s}-1}\right\}$ uma enumeração sem repetições de $\mathcal{A}_{s}$. Defina $J_{s(m)}=J_{s}^{m}$ para cada $m \in$ $\left\{0,1, \ldots, k_{s}-1\right\}$ e $J_{s} \frown_{(m)}=\emptyset$ para cada $m \in \omega \backslash\left\{0,1, \ldots, k_{s}-1\right\}$.

Para cada $n \in \omega$ considere o seguinte conjunto $A_{n}=\left\{s \in \omega^{n}: J_{s} \neq \emptyset\right\}$.

Afirmação 3.3.11. $0<\left|A_{n}\right|<\aleph_{0}$ para cada $n \in \omega$.

Demonstração. A demonstração será feita por indução em $n$.

- Suponha que $n=0$. Observe que $A_{0}=\left\{s \in \omega^{0}: J_{s} \neq \emptyset\right\}=\{\emptyset\}$. Logo, temos que $0<\left|A_{0}\right|<\aleph_{0}$.

- Fixe $n \in \omega$ arbitrário. Suponha que $0<\left|A_{n}\right|<\aleph_{0}$. Vamos estudar o caso $n+1$. Defina $\left|A_{n}\right|=k$. Fixe $i \in\{0,1, \ldots, k\}$ arbitrário. Por construção $\varepsilon_{s} \frown_{(i)} \in\left\{t \in \omega^{n+1}: J_{t} \neq \emptyset\right\}=A_{n+1}$. Logo, $0<\left|A_{n+1}\right|$. Defina $\left|\mathcal{A}_{s}{ }_{(i)}\right|=$ $k_{s}{ }_{(i)}<\aleph_{0}$. Note que $\left|A_{n+1}\right| \leq \sum_{i=0}^{k} k_{s} \frown_{(i)}$. Como $k_{s} \frown_{(i)}<\aleph_{0}$ para cada $i \in\{0,1, \ldots, k\}$ então $\sum_{i=0}^{k} k_{s}{ }_{(i)}<\aleph_{0}$. 
Defina $\delta_{n}=\min \left(A_{n}\right)$ para cada $n \in \omega$. Por construção temos que $\delta_{n}>0$ para cada $n \in \omega$. Por hipótese $X \subseteq \mathbb{K}$ é um conjunto fortemente nulo. Pela Proposição 3.3.7 temos que existe uma sequência de intervalos $\left(I_{n}\right)_{n \in \omega}$ tal que $\ell\left(I_{n}\right)<\delta_{n}$ para cada $n \in \omega$ e $X \subseteq \bigcap_{m \in \omega} \bigcup_{n>m} I_{n}$.

Por construção temos que para cada $n \in \omega$ existe $s_{n} \in{ }^{n+1} \omega$ tal que $\left(J_{s_{n}}+I_{n}\right) \cap C_{n}=$ $\emptyset$. Defina $f=\bigcup_{n \in \omega} s_{n}$. Observe que $f \in \omega^{\omega}$. Como $\left(J_{f_{\vdash n}}\right)_{n \in \omega}$ é uma sequência de intervalos fechados encaixantes em $\mathbb{K}$ e $\mathbb{K}$ é um espaço compacto então existe $x \in$ $\bigcap_{n \in \omega} J_{f_{\vdash n}}$ (aqui estamos usando a propriedade da interseção finita).

Iremos demonstrar que $\left[\left(\bigcap_{m \in \omega} \bigcup_{n>m} I_{n}\right)+x\right] \cap\left(\bigcup_{n \in \omega} C_{n}\right)=\emptyset$. De fato, suponha por absurdo que existe $y \in\left[\left(\bigcap_{m \in \omega} \bigcup_{n>m} I_{n}\right)+x\right] \cap\left(\bigcup_{n \in \omega} C_{n}\right)$. Então $y \in$ $\left(\bigcap_{m \in \omega} \bigcup_{n>m} I_{n}\right)+x$ e $y \in \bigcup_{n \in \omega} C_{n}$. Como $y \in\left(\bigcap_{m \in \omega} \bigcup_{n>m} I_{n}\right)+x$ então existe $z \in \bigcap_{m \in \omega} \bigcup_{n>m} I_{n}$ tal que $y=z+x$. Como $y \in \bigcup_{n \in \omega} C_{n}$ então existe $n_{0} \in \omega$ tal que $y \in C_{n_{0}}$. Como $z \in \bigcap_{m \in \omega} \bigcup_{n>m} I_{n}$ então $z \in \bigcup_{n>m} I_{n}$ para cada $m \in \omega$. Em particular $z \in I_{k_{0}}$ para algum $k_{0}>n_{0}$. Como $C_{n} \subseteq C_{n+1}$ para cada $n \in \omega$ então $C_{n_{0}} \subseteq C_{k_{0}}$. Portanto, $y \in C_{k_{0}}$. Então temos que $y \in\left(J_{f \backslash\left(k_{0}+1\right)}+I_{k_{0}}\right) \cap C_{k_{0}}$. Contradição.

Como $X \subseteq \bigcap_{m \in \omega} \bigcup_{n>m} I_{n}$ e $F \subseteq \bigcup_{n \in \omega} C_{n}$ então $(X+x) \cap F=\emptyset$.

Definição 3.3.12. Definimos a função $\varphi: \mathbb{R} \longrightarrow \mathbb{K}$ como sendo $\varphi(x)=x-\lfloor x\rfloor$ para cada $x \in \mathbb{R}$.

Lema 3.3.13. Se $X \subseteq \mathbb{R}$ é um conjunto fortemente nulo então $\varphi[X] \subseteq \mathbb{K}$ é um conjunto fortemente nulo de $\mathbb{K}$.

Demonstração. Defina $X_{n}=X \cap[n, n+1[$ para cada $n \in \mathbb{Z}$. Como $X$ é fortemente nulo e $X_{n} \subseteq X$ para cada $n \in \mathbb{Z}$ então $X_{n}$ é fortemente nulo para cada $n \in \mathbb{Z}$.

Iremos demonstrar que $\varphi\left[X_{n}\right] \subseteq \mathbb{K}$ é um conjunto fortemente nulo de $\mathbb{K}$ para cada $n \in \mathbb{Z}$. De fato, fixe $n \in \mathbb{Z}$ arbitrário. Seja $\left(\varepsilon_{j}\right)_{j \in \omega}$ uma sequência de números reais positivos. Como $X_{n} \subseteq \mathbb{R}$ é um conjunto fortemente nulo então existe uma sequência de intervalos $\left(I_{j}\right)_{j \in \omega}$ de $\mathbb{R}$ tal que $\ell\left(I_{j}\right) \leq \varepsilon_{j}$ para cada $j \in \omega$ e $X_{n} \subseteq \bigcup_{j \in \omega} I_{j}$. Note que $\ell\left(\varphi\left[I_{j}\right]\right)$ é um intervalo de $\mathbb{K}$ e $\ell\left(\varphi\left[I_{j}\right]\right) \leq \ell\left(I_{j}\right)$ para cada $j \in \omega$. Portanto, $\ell\left(\varphi\left[I_{j}\right]\right) \leq \varepsilon_{j}$ para cada $j \in \omega$. Observe que $\varphi\left[X_{n}\right] \subseteq \bigcup_{j \in \omega} \varphi\left[I_{j}\right]$.

Note que $\varphi[X]=\bigcup_{n \in \mathbb{Z}} \varphi\left[X_{n}\right]$. Como a reunião enumeravel de conjuntos fortemente nulos é um conjunto fortemente nulo então temos que $\varphi[X] \subseteq \mathbb{K}$ é um conjunto fortemente nulo. 
Lema 3.3.14. Se $F \subseteq \mathbb{R}$ é um fechado raro então $\varphi[F] \subseteq \mathbb{K}$ é um conjunto magro.

Demonstração. Defina $F_{n}=F \cap[n, n+1]$ para cada $n \in \mathbb{Z}$. Note que $F_{n} \subseteq \mathbb{R}$ é fechado em $\mathbb{R}$ para cada $n \in \mathbb{Z}$.

Fixe $n \in \mathbb{Z}$ arbitrário. Note que $\operatorname{int}_{\mathbb{R}}\left(F_{n}\right)=\emptyset$. De fato, suponha por absurdo que $\operatorname{int}_{\mathbb{R}}\left(F_{n}\right) \neq \emptyset$. Então existe $x \in F_{n}$ e $\varepsilon>0$ tal que $] x-\varepsilon, x+\varepsilon\left[\subseteq F_{n}\right.$. Como $F_{n} \subseteq F$ então $] x-\varepsilon, x+\varepsilon\left[\subseteq F\right.$. Logo, $\operatorname{int}_{\mathbb{R}}(F) \neq \emptyset$. Contradição, pois $F$ é um conjunto raro de $\mathbb{R}$.

Afirmação 3.3.15. $\varphi\left[F_{n}\right] \subseteq \mathbb{K}$ é fechado em $\mathbb{K}$ para cada $n \in \mathbb{Z}$.

Demonstração. Fixe $n \in \mathbb{Z}$ arbitrário. Defina $U=\mathbb{K} \backslash \varphi\left[F_{n}\right]$. Observe que $\varphi^{-1}[A]=$ $\mathbb{R} \backslash F_{n}$. Como $F_{n} \subseteq \mathbb{R}$ é fechado em $\mathbb{R}$ então $\mathbb{R} \backslash F_{n}$ é aberto em $\mathbb{R}$. Logo, $\varphi^{-1}[A]$ é aberto em $\mathbb{R}$. Portanto, $U=\mathbb{K} \backslash \varphi\left[F_{n}\right]$ é aberto em $\mathbb{K}$. Então $\varphi\left[F_{n}\right]$ é fechado em $\mathbb{K}$.

Afirmação 3.3.16. $\operatorname{int}_{\mathbb{K}}\left(\varphi\left[F_{n}\right]\right)=\emptyset$ para cada $n \in \mathbb{Z}$.

Demonstração. Suponha por absurdo que $\operatorname{int}_{\mathbb{K}}\left(\varphi\left[F_{n}\right]\right) \neq \emptyset$ para algum $n \in \mathbb{Z}$. Então existem $x \in \varphi\left[F_{n}\right]$ e $\left.\varepsilon \in\right] 0,1[$ tais que $] x-\varepsilon, x+\varepsilon\left[\subseteq \varphi\left[F_{n}\right]\right.$. Como $] x-\varepsilon, x+\varepsilon[\subseteq \mathbb{K}$ é aberto em $\mathbb{K}$ e $\varphi: \mathbb{R} \longrightarrow \mathbb{K}$ é uma função contínua então $\varphi^{-1}[] x-\varepsilon, x+\varepsilon[] \subseteq \mathbb{R}$ é aberto em $\mathbb{R}$. Como $] x-\varepsilon, x+\varepsilon\left[\neq \emptyset\right.$ então $\varphi^{-1}[] x-\varepsilon, x+\varepsilon[] \neq \emptyset$. Note que $\varphi^{-1}[] x-\varepsilon, x+\varepsilon[] \subseteq F_{n}$. Como $\varphi^{-1}[] x-\varepsilon, x+\varepsilon[] \subseteq \mathbb{R}$ é um aberto não-vazio de $\mathbb{R}$ então existem $y \in \varphi^{-1}[] x-\varepsilon, x+\varepsilon[$ e $\delta>0$ tais que $] y-\delta, y+\delta\left[\subseteq \varphi^{-1}[] x-\varepsilon, x+\varepsilon[]\right.$. Como $\varphi^{-1}[] x-\varepsilon, x+\varepsilon[] \subseteq F_{n}$ então $] y-\delta, y+\delta\left[\subseteq F_{n} . \operatorname{Logo} \operatorname{int}_{\mathbb{R}}\left(F_{n}\right) \neq \emptyset\right.$. Contradição.

Então temos que $\varphi\left[F_{n}\right]$ é um fechado raro de $\mathbb{K}$ para cada $n \in \mathbb{Z}$. Como $\varphi[F]=$ $\bigcup_{n \in \mathbb{Z}} \varphi\left[F_{n}\right]$ então $F$ é um conjunto magro de $\mathbb{K}$.

Corolário 3.3.17. Se $X \subseteq \mathbb{R}$ é um conjunto magro de $\mathbb{R}$ então $\varphi[X]$ é um conjunto magro de $\mathbb{K}$.

Demonstração. Considere $\left(X_{n}\right)_{n \in \omega}$ tal que $X_{n} \subseteq \mathbb{R}$ é um fechado raro para cada $n \in \omega$ e $X=\bigcup_{n \in \omega} X_{n}$. Pelo Lema 3.3.14 temos que $\varphi\left[X_{n}\right] \subseteq \mathbb{K}$ é magro em $\mathbb{K}$ para cada $n \in \omega$. Note que $\varphi[X]=\bigcup_{n \in \omega} \varphi\left[X_{n}\right]$. Como a reunião enumerável de conjuntos magros é um conjunto magro então $\varphi[X]$ é um conjunto magro.

Teorema 3.3.18. Se $X \subseteq \mathbb{R}$ é um conjunto fortemente nulo então para cada conjunto magro $F \subseteq \mathbb{R}$ existe $x \in \mathbb{R}$ tal que $(X+x) \cap F=\emptyset$. 
Demonstração. Pelo Lema 3.3.13 temos que $\varphi[X] \subseteq \mathbb{K}$ é um conjunto fortemente nulo. Pelo Corolário 3.3.17 temos que $\varphi[F] \subseteq \mathbb{K}$ é um conjunto magro de $\mathbb{K}$. Pelo Teorema 3.3.10 temos que existe $x \in \mathbb{K}$ tal que $(\varphi[X]+x) \cap \varphi[F]=\emptyset$. Note que $(X+x) \cap F=\emptyset$. De fato, suponha por absurdo que existe $y \in(X+x) \cap F$. Então $y \in X+x$ e $y \in F$. Como $y \in X+x$ então existe $x^{\prime} \in X$ tal que $y=x^{\prime}+x$. Observe que:

$$
\varphi(y)=\varphi\left(x^{\prime}+x\right)=\varphi\left(x^{\prime}\right)+\varphi(x)=\varphi\left(x^{\prime}\right)+x .
$$

Como $\varphi\left(x^{\prime}\right) \in \varphi[X]$ então $\varphi(y)=\varphi\left(x^{\prime}\right)+x \in \varphi[X]+x$. Como $y \in F$ então $\varphi(y) \in$ $\varphi[F]$. Logo, $\varphi(y) \in(\varphi[X]+x) \cap \varphi[F]$. Contradição, pois $(\varphi[X]+x) \cap \varphi[F]=\emptyset$.

Corolário 3.3.19 (Galvin-Mycielski-Solovay [6]). Seja $X \subseteq \mathbb{R}$. São equivalentes:

1. $X \in \mathcal{S N}$

2. para cada $F \in \mathcal{M}$ existe $x \in \mathbb{R}$ tal que $(X+x) \cap F=\emptyset$

3. $X+F \neq \mathbb{R}$ para cada $F \in \mathcal{M}$. 


\subsection{Conjectura de Borel}

Sabemos que, todo subconjunto enumerável de $\mathbb{R}$ é fortemente nulo. A Conjectura de Borel, abreviada por $B C$, é a afirmação:

Conjectura de Borel (BC). Todo subconjunto fortemente nulo de $\mathbb{R}$ é enumerável.

Em 1938 Sierpiński [15] provou a consistência relativa de $\neg B C$ mostrando que, se vale $C H$, então existe um subconjunto não enumerável de $\mathbb{R}$ que é fortemente nulo (conjunto de Luzin).

Em 1976, Laver, usando a técnica de forcing, mostrou que é consistente com ZFC que todo subconjunto fortemente nulo de $\mathbb{R}$ é enumerável.

Com tudo, hoje sabemos que a Conjectura de Borel é uma afirmação independente de ZFC.

Definição 3.4.1. Seja $X \subseteq \mathbb{R}$. Dizemos que $X$ é um conjunto de Luzin se $|X|>\aleph_{0}$ e $|X \cap F| \leq \aleph_{0}$ para cada $F \in \mathcal{M}$.

Teorema 3.4.2. Assuma CH. Existe um conjunto de Luzin.

Demonstração. Pelo Lema 2.2.37, existem $\mathfrak{c}$ subconjuntos fechados raros em $\mathbb{R}$. Seja $\left\{F_{\alpha}: \alpha<\omega_{1}\right\}$ uma indexação de todos os fechados raros em $\mathbb{R}$.

Tome $x_{0} \notin F_{0}$. Existe $x_{\alpha} \in \mathbb{R} \backslash\left(\left\{x_{\beta}: \beta<\alpha\right\} \cup \bigcup_{\beta<\alpha} F_{\beta}\right)$ para cada $\alpha<\omega_{1}$. De fato, observe que:

$$
\left\{x_{\beta}: \beta<\alpha\right\} \cup\left(\bigcup_{\beta<\alpha} F_{\beta}\right)=\left(\left(\bigcup_{\beta<\alpha}\left\{x_{\beta}\right\}\right) \cup\left(\bigcup_{\beta<\alpha} F_{\beta}\right)\right) \in \mathcal{M} .
$$

O complementar de um conjunto magro é um aberto denso. Em particular, temos que $\left(\mathbb{R} \backslash\left(\left\{x_{\beta}: \beta<\alpha\right\} \cup \bigcup_{\beta<\alpha} F_{\beta}\right)\right) \neq \emptyset$. Portanto, existe $x_{\alpha} \in \mathbb{R} \backslash\left(\left\{x_{\beta}: \beta<\alpha\right\} \cup \bigcup_{\beta<\alpha} F_{\beta}\right)$.

Defina $L=\left\{x_{\alpha}: \alpha<\omega_{1}\right\}$. Iremos demonstrar que $L$ é um conjunto de Luzin. Com efeito, por construção, $|L|>\aleph_{0}$. Considere $F \in \mathcal{M}$ arbitrário. Seja $\Gamma \subseteq\left\{\xi: \xi<\omega_{1}\right\}$ tal que $|\Gamma| \leq \aleph_{0}$ e $F=\bigcup_{\alpha \in \Gamma} F_{\alpha}$. Note que para cada $\alpha<\omega_{1}$ temos que $L \cap F_{\alpha} \subseteq$ $\left\{x_{\beta}: \beta \leq \alpha\right\}$. De fato, seja $x_{\beta} \in L \operatorname{com} \beta>\alpha$, temos que $x_{\beta} \notin F_{\alpha}$ pois por construção $x_{\beta} \in \mathbb{R} \backslash \bigcup_{\gamma<\beta} F_{\gamma}$.

Como $\left\{x_{\beta}: \beta \leq \alpha\right\}$ é enumerável temos que $L \cap F_{\alpha}$ é enumerável e, portanto, temos que: 


$$
L \cap F=L \cap \bigcup_{\alpha \in \Gamma} F_{\alpha}=\bigcup_{\alpha \in \Gamma} L \cap F_{\alpha} \subseteq \bigcup_{\alpha \in \Gamma}\left\{x_{\beta}: \beta \leq \alpha\right\} .
$$

Como $\omega_{1}$ é o primeiro ordinal não enumerável temos que $\left\{x_{\beta}: \beta \leq \alpha\right\}$ é enumerável para cada $\alpha<\omega_{1}$ e, além disso, a reunião enumerável de conjuntos enumeráveis é enumerável, portanto, $\bigcup_{\alpha \in \Gamma}\left\{x_{\beta}: \beta \leq \alpha\right\}$ é enumerável. Como todo subconjunto de um conjunto enumerável também é enumerável, $F \cap L$ é enumerável. Logo, $L$ é um conjunto de Luzin.

Teorema 3.4.3 (Sierpiński [15]). Seja $X \subseteq \mathbb{R}$ um conjunto de Luzin. Então $X$ é fortemente nulo.

Demonstração. Considere $X \subseteq \mathbb{R}$ um conjunto de Luzin. Sejam $\left(\varepsilon_{n}\right)_{n \in \omega}$ uma sequência de reais positivos e $\left\{q_{n}: n \in \omega\right\}$ uma enumeração de $\mathbb{Q}$.

Note que $\left.D=\bigcup_{n \in \omega}\right] q_{n}-\frac{\varepsilon_{2 n}}{2}, q_{n}+\frac{\varepsilon_{2 n}}{2}[$ é aberto denso em $\mathbb{R}$ e, portanto, $\mathbb{R} \backslash D$ é magro em $\mathbb{R}$. Como $X$ é um conjunto de Luzin, temos que $X \cap(\mathbb{R} \backslash D)$ é enumerável. Seja $\left\{x_{n}: n \in \omega\right\}$ uma enumeração para $X \cap(\mathbb{R} \backslash D)$. Então, temos que

$X \subseteq((X \cap D) \cup(X \cap(\mathbb{R} \backslash D))) \subseteq\left(\bigcup_{n \in \omega}\right] q_{n}-\frac{\varepsilon_{2 n}}{2}, q_{n}+\frac{\varepsilon_{2 n}}{2}[) \cup\left(\bigcup_{n \in \omega}\right] x_{n}-\frac{\varepsilon_{2 n+1}}{2}, x_{n}+\frac{\varepsilon_{2 n+1}}{2}[)$

Corolário 3.4.4. $C H \longrightarrow \neg B C$.

Corolário 3.4.5. $\neg B C$ é consistente com ZFC. 


\subsection{Teorema de Goldstern-Judah-Shelah}

Definição 3.5.1. Seja $\kappa$ um cardinal não enumerável. Dizemos que $\mathcal{F} \subseteq \omega^{\omega}$ é uma família $\kappa$-fortemente ilimitada se $\kappa \leq|\mathcal{F}|$ e para cada $g \in \omega^{\omega}$ temos $\mid\left\{f \in \mathcal{F}: f \leq^{*}\right.$ $g\} \mid<\kappa$.

Proposição 3.5.2. Seja $\kappa$ um cardinal não enumerável. Se $\mathcal{F} \subseteq \omega^{\omega}$ é uma família $\kappa$-fortemente ilimitada então $\mathcal{F}$ é uma família ilimitada.

Demonstração. Fixe $\mathcal{F} \subseteq \omega^{\omega}$ família $\kappa$-fortemente ilimitada. Suponha por absudo que $\mathcal{F}$ não é ilimitada. Então existe $g \in \omega^{\omega}$ tal que $f \leq^{*} g$ para cada $f \in \mathcal{F}$. Então temos que $\left|\left\{f \in \mathcal{F}: f \leq^{*} g\right\}\right|=\kappa$.

Como $\mathcal{F} \subseteq \omega^{\omega}$ família $\kappa$-fortemente ilimitada então pela Definição 3.5.1 temos $\left|\left\{f \in \mathcal{F}: f \leq^{*} g\right\}\right|<\kappa$. Logo, $\kappa<\kappa$. Contradição.

Proposição 3.5.3. Se $\left\{f_{\alpha}: \alpha<\mathfrak{d}\right\} \subseteq \omega^{\omega}$ então existe $\left\{g_{\alpha}: \alpha<\mathfrak{d}\right\} \subseteq \omega^{\omega}$ tal que $f_{\alpha} \leq^{*} g_{\alpha}$ para cada $\alpha<\mathfrak{d}$ e $g_{\alpha} \mathbb{Z}^{*} g_{\beta}$ para cada $\beta<\alpha<\mathfrak{d}$.

Demonstração. Para obter a família $\left\{g_{\alpha}: \alpha<\mathfrak{d}\right\} \subseteq \omega^{\omega}$ satisfazendo as propriedades desejadas iremos proceder por indução transfinita:

- Defina $g_{0}=f_{0}$;

- Fixe $\gamma<\mathfrak{d}$ arbitrário. Suponha construído $g_{\alpha} \in \omega^{\omega}$ para cada $\alpha<\gamma$ tal que $f_{\alpha} \leq^{*} g_{\alpha}$ para cada $\alpha<\gamma$ e $g_{\alpha} \not^{*} g_{\beta}$ para cada $\beta<\alpha<\gamma$. Queremos construir $g_{\gamma} \in \omega^{\omega}$ tal que $f_{\gamma} \leq^{*} g_{\gamma}$ e $g_{\gamma} \mathbb{Z}^{*} g_{\beta}$ para cada $\beta<\gamma$. Considere $\left\{g_{\alpha} \in \omega^{\omega}: \alpha<\gamma\right\}$. Como $\gamma<\mathfrak{d}$ então $\left\{g_{\alpha} \in \omega^{\omega}: \alpha<\gamma\right\}$ não é uma família dominante. Logo, existe $h_{\gamma} \in \omega^{\omega}$ tal que $h_{\gamma} \mathbb{Z}^{*} g_{\alpha}$ para cada $\alpha<\gamma$. Considere $g_{\gamma} \in \omega^{\omega}$ dada por $g_{\gamma}(n)=\max \left\{h_{\gamma}(n), f_{\gamma}(n)\right\}$ para cada $n \in \omega$. Note que $h_{\gamma}, f_{\gamma} \leq^{*} g_{\gamma}$. Note que $g_{\gamma} \mathbb{Z}^{*} g_{\xi}$ para cada $\xi<\gamma$, pois caso contrário teríamos $h_{\gamma} \leq^{*} g_{\gamma} \leq^{*} g_{\xi}$. O que contraria a escolha de $h_{\gamma}$.

Teorema 3.5.4. Existe $\mathcal{G} \subseteq \omega^{\omega}$ tal que $\mathcal{G}$ é $\mathfrak{d}$-fortemente ilimitada. 
Demonstração. Seja $\left\{f_{\alpha}: \alpha<\mathfrak{d}\right\} \subseteq \omega^{\omega}$ uma família dominante. Considere $\left\{g_{\alpha} \in \omega^{\omega}\right.$ : $\alpha<\mathfrak{d}\}$ dada pela Proposição 3.5.3. Defina $\mathcal{G}=\left\{g_{\alpha} \in \omega^{\omega}: \alpha<\mathfrak{d}\right\}$. Observe que $\mathcal{G}$ é uma família dominante, pois $f_{\alpha} \leq^{*} g_{\alpha}$ para cada $\alpha<\mathfrak{d}$. Portanto, $|\mathcal{G}|=\mathfrak{d}$.

Afirmamos que $\left|\left\{g \in \mathcal{G}: g \leq^{*} f\right\}\right|<\mathfrak{d}$ para cada $f \in \omega^{\omega}$. De fato, fixe $f \in \omega^{\omega}$ arbitrária. Como $\left\{g_{\alpha}: \alpha<\mathfrak{d}\right\} \subseteq \omega^{\omega}$ é uma família dominante então existe $\beta<\mathfrak{d}$ tal que $f \leq^{*} g_{\beta}$. Por hipótese, $g_{\alpha} \mathbb{Z}^{*} g_{\beta}$ para cada $\beta<\alpha<\mathfrak{d}$. Então $\left\{g \in \mathcal{G}: g \leq^{*} f\right\} \subseteq$ $\left\{g \in \mathcal{G}: g \leq^{*} g_{\beta}\right\} \subseteq\left\{g_{\alpha}: \alpha \leq \beta\right\}$. Como $\left|\left\{g_{\alpha}: \alpha \leq \beta\right\}\right| \leq|\beta|$ então $\mid\left\{g \in \mathcal{G}: g \leq^{*}\right.$ $\left.g_{\beta}\right\} \mid \leq \beta$. Logo, temos $\left|\left\{g \in \mathcal{G}: g \leq^{*} f\right\}\right| \leq\left|\left\{g \in \mathcal{G}: g \leq^{*} g_{\beta}\right\}\right| \leq|\beta|<\mathfrak{d}$.

Proposição 3.5.5. Se $\left\{f_{\alpha}: \alpha<\mathfrak{b}\right\} \subseteq \omega^{\omega}$ então existe $\left\{g_{\alpha}: \alpha<\mathfrak{b}\right\} \subseteq \omega^{\omega}$ tal que $f_{\alpha} \leq^{*} g_{\alpha}$ para cada $\alpha<\mathfrak{b}$ e $g_{\alpha} \leq^{*} g_{\beta}$ para cada $\alpha<\beta<\mathfrak{b}$.

Demonstração. Para obter a família $\left\{g_{\alpha}: \alpha<\mathfrak{b}\right\} \subseteq \omega^{\omega}$ satisfazendo as propriedades desejadas iremos proceder por indução transfinita:

- $g_{0}=f_{0}$

- Fixe $\gamma<\mathfrak{b}$ arbitrário. Suponha construído $g_{\alpha} \in \omega^{\omega}$ para cada $\alpha<\gamma$ tal que $f_{\alpha} \leq^{*} g_{\alpha}$ para cada $\alpha<\gamma$ e $g_{\alpha} \leq^{*} g_{\beta}$ para cada $\alpha<\beta<\gamma$. Queremos construir $g_{\gamma} \in \omega^{\omega}$ tal que $f_{\gamma} \leq^{*} g_{\gamma}$ e $g_{\beta} \leq^{*} g_{\gamma}$ para cada $\beta<\gamma$. Como $\mid\left\{g_{\alpha} \in \omega^{\omega}: \alpha<\right.$ $\gamma\}|\leq| \gamma \mid<\mathfrak{b}$ então $\left\{g_{\alpha}: \alpha<\gamma\right\} \subseteq \omega^{\omega}$ não é uma família ilimitada. Então existe $h_{\gamma} \in \omega^{\omega}$ tal que $g_{\alpha} \leq^{*} h_{\gamma}$ para cada $\alpha<\gamma$. Considere $g_{\gamma} \in \omega^{\omega}$ dada por $g_{\gamma}(n)=\max \left\{h_{\gamma}(n), f_{\gamma}(n)\right\}$ para cada $n \in \omega$. Note que $h_{\gamma}, f_{\gamma} \leq^{*} g_{\gamma}$. Pela escolha de $g_{\gamma} \in \omega^{\omega}$ temos que $g_{\alpha} \leq^{*} g_{\gamma}$ para cada $\alpha<\gamma$.

Teorema 3.5.6. Existe $\mathcal{G} \subseteq \omega^{\omega}$ tal que $\mathcal{G}$ é $\mathfrak{b}$-fortemente ilimitada.

Demonstração. Seja $\left\{f_{\alpha}: \alpha<\mathfrak{b}\right\}$ uma família ilimitada. Considere $\left\{g_{\alpha}: \alpha<\mathfrak{b}\right\}$ dada pela Proposição 3.5.5. Defina $\mathcal{G}=\left\{g_{\alpha}: \alpha<\mathfrak{b}\right\}$. Como $f_{\alpha} \leq^{*} g_{\alpha}$ para cada $\alpha<\mathfrak{b}$ então $\mathcal{G}$ é uma família ilimitada. Logo, $|\mathcal{G}|=\mathfrak{b}$.

Iremos demonstrar que $\left|\left\{g \in \mathcal{G}: g \leq^{*} f\right\}\right|<\mathfrak{b}$ para cada $f \in \omega^{\omega}$. Com efeito, fixe $f \in \omega^{\omega}$ arbitrária. Como $\left\{f_{\alpha}: \alpha<\mathfrak{b}\right\} \subseteq \omega^{\omega}$ é uma família ilimitada então existe $\beta<\mathfrak{b}$ tal que $f_{\beta} \not^{*} f$. Pela Proposição 3.5 .5 temos que $f_{\alpha} \leq^{*} g_{\alpha}$ para cada $\alpha<\mathfrak{b}$. Então, $g_{\beta} \mathbb{Z}^{*} f$. Por hipótese, $g_{\alpha} \leq^{*} g_{\xi}$ para cada $\alpha<\xi<\mathfrak{b}$. Então $\left\{g \in \mathcal{G}: g \leq^{*} f\right\} \subseteq\left\{g \in \mathcal{G}: g \leq^{*} g_{\beta}\right\} \subseteq\left\{g_{\alpha}: \alpha \leq \beta\right\}$. Como $\left|\left\{g_{\alpha}: \alpha \leq \beta\right\}\right| \leq|\beta|$ então $\left|\left\{g \in \mathcal{G}: g \leq^{*} g_{\beta}\right\}\right| \leq \beta$. Logo, temos que $\left|\left\{g \in \mathcal{G}: g \leq^{*} f\right\}\right| \leq \beta<\mathfrak{b}$. 
Teorema 3.5.7 (Goldstern-Judah-Shelah [8]). Assuma que existe uma familia $\kappa$-fortemente ilimitada para cada $\kappa \leq \mathfrak{c} . S e[\mathbb{R}]^{<\kappa} \subseteq \mathcal{S N}$ então existe $X \subseteq \mathbb{R}$ tal que $X$ é fortemente nulo e $|X|=\kappa$.

Demonstração. Sejam $\mathcal{F}=\left\{f_{\alpha} \in \omega^{\omega}: \alpha<\kappa\right\}$ é uma família $\kappa$-fortemente ilimitada e $\left\{q_{n}: n \in \omega\right\}$ uma enumeração sem repetições de $\mathbb{Q} \cap[0,1]$. Seja $\psi: \omega^{\omega} \longrightarrow \mathbb{P} \cap[0,1]$ um homeomorfismo. Iremos demonstrar que $\psi[\mathcal{F}] \subseteq[0,1]$ é fortemente nulo.

Fixe $\left(\varepsilon_{n}\right)_{n \in \omega}$ sequência de números reais positivos. Observe que $\left.U=\bigcup_{n \in \omega}\right] q_{n}-\varepsilon_{2 n}, q_{n}+\varepsilon_{2 n}[\cap$ $[0,1]$ é aberto em $[0,1]$. Considere $F=[0,1] \backslash U \subseteq[0,1]$. Como $U$ é aberto em $[0,1]$ então $F$ é fechado em $[0,1]$. Como $[0,1]$ é um espaço de Hausdorff compacto então $F$ é compacto. Note que $\psi^{-1}[F] \subseteq \omega^{\omega}$ é compacto. Sabemos que existe $g \in \omega^{\omega}$ tal que:

$$
\psi^{-1}[F] \subseteq\left\{f \in \omega^{\omega}: f \leq^{*} g\right\}
$$

Note que $\psi^{-1}[F]=\left\{f \in \omega^{\omega}: \psi(f) \notin U\right\}$. Como $\{f \in \mathcal{F}: \psi(f) \notin U\} \subseteq \psi^{-1}[F]$ então temos $\{f \in \mathcal{F}: \psi(f) \notin U\} \subseteq\left\{f \in \mathcal{F}: f \leq^{*} g\right\}$. Como $\left\{f_{\alpha} \in \omega^{\omega}: \alpha<\kappa\right\}$ é uma família $\kappa$-fortemente ilimitada então $\left|\left\{f \in \mathcal{F}: f \leq^{*} g\right\}\right|<\kappa$ e, portanto, $|\{f \in \mathcal{F}: \psi(f) \notin U\}|<\kappa$.

Como $\psi: \omega^{\omega} \longrightarrow \omega^{\omega}$ é uma função bijetora então $\left|\psi\left[\psi^{-1}[F]\right]\right|=|F|<\kappa$. Por hipótese $F \subseteq[0,1]$ é fortemente nulo. Então existe uma sequência de números reais $\left(r_{n}\right)_{n \in \omega}$ tal que $\left.F \subseteq \bigcup_{n \in \omega}\right] r_{n}-\varepsilon_{2 n+1}, r_{n}+\varepsilon_{2 n+1}[$. Observe que:

$$
\begin{aligned}
\psi[\mathcal{F}] & \subseteq U \cup F \\
& \subseteq\left(\bigcup_{n \in \omega}\right] q_{n}-\varepsilon_{2 n}, q_{n}+\varepsilon_{2 n}[) \cup\left(\bigcup_{n \in \omega}\right] r_{n}-\varepsilon_{2 n+1}, r_{n}+\varepsilon_{2 n+1}[) .
\end{aligned}
$$

Corolário 3.5.8. Se $[\mathbb{R}]^{<\mathfrak{d}} \subseteq \mathcal{S N}$ então existe $X \subseteq \mathbb{R}$ tal que $X$ é fortemente nulo e $|X|=\mathfrak{d}$.

Demonstração. Pelo Teorema 3.5.4 temos que existe $\mathcal{G}=\left\{g_{\alpha} \in \omega^{\omega}: \alpha<\mathfrak{d}\right\}$ família d-fortemente ilimitada. Então basta proceder como na demonstração do Teorema 3.5.7.

Corolário 3.5.9. Se $[\mathbb{R}]^{<\mathfrak{b}} \subseteq \mathcal{S N}$ então existe $X \subseteq \mathbb{R}$ tal que $X$ é fortemente nulo e $|X|=\mathfrak{b}$. 
Demonstração. Pelo Teorema 3.5 .6 temos que existe $\mathcal{G}=\left\{g_{\alpha} \in \omega^{\omega}: \alpha<\mathfrak{b}\right\}$ família $\mathfrak{b}$ - fortemente ilimitada. Então basta proceder como na demonstração do Teorema 3.5.7.

Corolário 3.5.10. Se $\mathfrak{b}=\aleph_{1}$ então existe $X \subseteq \mathbb{R}$ fortemente nulo tal que $X$ é não enumerável.

Demonstração. Decorre diretamente do Corolário 3.5.9. 


\section{Capítulo 4}

\section{Equivalências}

\subsection{Espaços Métricos e a Conjectura de Borel}

Galvin conjecturou (Carlson, [5]) que se vale a Conjectura de Borel então todo espaço métrico fortemente nulo é enumerável. Nesta seção iremos verificar tal conjectura.

Começaremos enunciando dois resultados que serão utilizados mais adiante. Suas demonstrações serão omitidas, pois fogem do escopo desse trabalho.

Teorema 4.1.1. Sejam $z_{0} \in \mathbb{C}$ e $f: \mathbb{C} \longrightarrow \mathbb{C}$. Se existe $R \in \mathbb{R}_{>0}$ tal que $f(z)=$ $\sum_{n \in \omega} a_{n}\left(z-z_{0}\right)^{n}$ para cada $z \in\left\{w \in \mathbb{C}:\left|w-z_{0}\right|<R\right\}$ então $\sum_{n \in \omega} a_{n}\left(z-z_{0}\right)^{n}$ é a espansão em série de Taylor de $f$.

Teorema 4.1.2. Sejam $f: \mathbb{C} \longrightarrow \mathbb{C}$ e $z_{0} \in \mathbb{C}$. Suponha que:

1. $f$ é analítica em $z_{0}$ e $f\left(z_{0}\right)=0$

2. Se $V$ uma vizinhança aberta $z_{0}$ então $f(w) \neq 0$ para cada $w \in V \backslash\left\{z_{0}\right\}$.

Existe $\varepsilon>0$ tal que $f(z) \neq 0$ para cada $z \in \mathbb{C} \backslash B_{\varepsilon}\left(z_{0}\right)$.

Definição 4.1.3. Sejam $\left(X, d_{X}\right)$ e $\left(Y, d_{Y}\right)$ espaços métricos. Dizemos que $f: X \longrightarrow Y$ é uma função lipschitziana se existe um número real não-negativo $L$ (constante de Lipschitz) tal que $d_{Y}(f(x), f(y)) \leq L \cdot d_{X}(x, y)$ para cada $x, y \in X$.

Lema 4.1.4. Se $(X, d)$ é um espaço métrico separável tal que $|X|<\mathfrak{c}$ então existe uma função $f: X \longrightarrow \mathbb{R}$ injetora e lipschitziana. 
Demonstração. Como $X$ é separável existe $D \subseteq X$ denso enumerável. Seja $\left\{x_{n}: n \in \omega\right\}$ uma enumeração para $D$ sem repetições. Seja $\rho: X \times X \longrightarrow \mathbb{R}_{\geq 0}$ dada por $\rho(x, y)=$ $\min \{1, d(x, y)\}$. Note que $\rho$ é uma métrica equivalente a métrica $d$. Além disso, $\rho$ é limitada.

Afirmação 4.1.5. Se $x, y \in X$ e $x \neq y$ então existe $n \in \omega$ tal que $\rho\left(x, x_{n}\right) \neq \rho\left(y, x_{n}\right)$.

Demonstração. Suponha por absurdo que $\rho\left(x, x_{n}\right)=\rho\left(y, x_{n}\right)$ para cada $n \in \omega$. Defina $\varepsilon=\frac{\rho(x, y)}{2}$. Observe que $y \notin B_{\varepsilon}(x)$. Como $d$ e $\rho$ são métricas equivalentes e $D \subseteq X$ é denso em $X$ então existe $x_{n} \in B_{\frac{\varepsilon}{2}}(x)$. Usando a desigualdade triangular temos que $\rho(x, y) \leq \rho\left(x, x_{n}\right)+\rho\left(x_{n}, y\right)$. Como $x_{n} \in B_{\frac{\varepsilon}{2}}(x)$ então $\rho\left(x_{n}, x\right)=\rho\left(x, x_{n}\right)<\frac{\varepsilon}{2}$. Logo, temos que:

$$
\rho\left(x, x_{n}\right)+\rho\left(x_{n}, x\right)<\frac{\varepsilon}{2}+\frac{\varepsilon}{2}<\varepsilon .
$$

Logo, $y \in B_{\varepsilon}(x)$. Contradição.

Fixe $x \in X$. Defina $f_{x}: \mathbb{C} \longrightarrow \mathbb{C}$ como sendo:

$$
f_{x}(z)=\sum_{n \in \omega}\left(\frac{\rho\left(x, x_{n}\right)}{n !}\right) z^{n} .
$$

Note que $f_{x}$ está bem definida, pois $\rho$ é uma métrica limitada. Por definição, temos que $f_{x}$ é uma função analítica.

Afirmação 4.1.6. Se $x, y \in X$ e $x \neq y$ então $f_{x} \neq f_{y}$.

Demonstração. Suponha que $f_{x}(z)=f_{y}(z)$ para cada $z \in \mathbb{C}$. Usando as definições de $f_{x}, f_{y}$, igualando a zero e agrupando os somatórios obtemos:

$$
\sum_{n \in \omega}\left(\frac{\rho\left(x, x_{n}\right)-\rho\left(x_{n}, y\right)}{n !}\right) z^{n}=0 .
$$

Então $\frac{\rho\left(x, x_{n}\right)-\rho\left(x_{n}, y\right)}{n !}=0$ para cada $n \in \omega$ (Teorema 4.1.1. Logo, $\rho\left(x, x_{n}\right)=$ $\rho\left(x_{n}, y\right)$ para cada $n \in \omega$. O que contradiz a Afirmação 4.1.5.

Iremos demonstrar que existe $r \in \mathbb{R}_{>0}$ tal que $f_{x}(r) \neq f_{y}(r)$ para cada $x, y \in X$ com $x \neq y$. De fato, considere $x, y \in X$ tais que $x \neq y$. Pela Afirmação 4.1 .6 temos que $f_{x} \neq f_{y}$. Como $f_{x}$ e $f_{y}$ são funções analíticas então $\left|\left\{z \in \mathbb{C}: f_{x}(z)=f_{y}(z)\right\}\right| \leq \aleph_{0}$ 
(Teorema 4.1.2. Denote por $W$ o conjunto $\bigcup_{x, y \in X}\left\{z \in \mathbb{C}: f_{x}(z)=f_{y}(z)\right.$ e $\left.x \neq y\right\}$. Observe que:

$$
|W|=|X| \cdot \aleph_{0}=\max \left\{|X|, \aleph_{0}\right\} .
$$

Como $|X|<\mathfrak{c}$ e $|\mathbb{R}|=\left|\mathbb{R}_{>0}\right|=\mathfrak{c}$ então $\mathbb{R}_{>0} \backslash W \neq \emptyset$. Portanto, existe $r \in \mathbb{R}_{>0} \backslash W$ tal que $f_{x}(r) \neq f_{y}(r)$ para cada $x, y \in X$.

Considere $f: X \longrightarrow \mathbb{R}$ dada por $f(x)=f_{x}(r)$ para cada $x \in X$. Por construção, temos que $f$ é injetora. Demonstraremos que a função $f$ é lipschitziana. Com efeito, sejam $x, y \in X \operatorname{com} x \neq y$. Então temos que:

$$
\begin{aligned}
|f(x)-f(y)| & =\left|f_{x}(r)-f_{y}(r)\right| ; \\
& =\left|\sum_{n \in \omega}\left(\frac{\rho\left(x, x_{n}\right)}{n !}\right) r^{n}-\sum_{n \in \omega}\left(\frac{\rho\left(y, x_{n}\right)}{n !}\right) r^{n}\right| \\
& =\left|\sum_{n \in \omega}\left(\frac{\rho\left(x, x_{n}\right)-\rho\left(y, x_{n}\right)}{n !}\right) r^{n}\right| \\
& \leq \sum_{n \in \omega}\left|\left(\frac{\rho\left(x, x_{n}\right)-\rho\left(y, x_{n}\right)}{n !}\right) r^{n}\right| \\
& \stackrel{r>0}{=} \sum_{n \in \omega}\left(\frac{\left|\rho\left(x, x_{n}\right)-\rho\left(y, x_{n}\right)\right|}{n !}\right) r^{n} \\
& \leq \sum_{n \in \omega}\left(\frac{\rho(x, y)}{n !}\right) r^{n} \\
& \leq \sum_{n \in \omega}\left(\frac{d(x, y)}{n !}\right) r^{n} \\
& =\left(\sum_{n \in \omega} \frac{r^{n}}{n !}\right) \cdot d(x, y) \\
& =e^{r} \cdot d(x, y) .
\end{aligned}
$$

Portanto, $f$ é uma função lipschitiziana com constante de Lipschitz igual a $e^{r}$.

Definição 4.1.7. Suponha que $\alpha$ é um número real e que $A \subseteq \mathbb{R}$. Denotamos por $\alpha \cdot A$ o conjunto $\{\alpha \cdot x: x \in A\}$. 
Observação 4.1.8. Suponha que $\alpha$ é um número real não-negativo e que $A \subseteq \mathbb{R}$ é um conjunto não-vazio limitado superiormente. Então $\sup (\alpha \cdot A)=\alpha \cdot \sup (A)$.

Lema 4.1.9. Sejam $\left(X, d_{X}\right)$ e $\left(Y, d_{Y}\right)$ espaços métricos. Se $f: X \longrightarrow Y$ é uma função lipschtziana e $A \subseteq X$ é limitado então $\operatorname{diam}(f[A]) \leq L \cdot \operatorname{diam}(A)$, sendo que $L$ é a constante de Lipschitz de $f$.

Demonstração. Pela definição de diâmetro de um conjunto temos:

$$
\operatorname{diam}(f[A])=\sup \left\{d_{Y}(f(x), f(y)): x, y \in A\right\}
$$

Como $f: X \longrightarrow Y$ é uma função lipschitziana (Definição 4.1.3) então existe um número real não-negativo $L$ tal que $d_{Y}(f(x), f(y)) \leq L \cdot d_{X}(x, y)$ para cada $x, y \in X$. Em particular, temos que $d_{Y}(f(x), f(y)) \leq L \cdot d_{X}(x, y)$ para cada $x, y \in A$.

Tomando o supremo em ambos os lados na desigualdade acima, obtemos:

$$
\begin{aligned}
\operatorname{diam}(f[A])=\sup \left\{d_{Y}(f(x), f(y)): x, y \in A\right\} & \leq \sup \left\{L \cdot d_{X}(x, y): x, y \in A\right\} \\
& \stackrel{4.1 .8}{=} L \cdot \sup \left\{d_{X}(x, y): x, y \in A\right\} \\
& =L \cdot \operatorname{diam}(A) .
\end{aligned}
$$

Proposição 4.1.10. Sejam $\left(X, d_{X}\right),\left(Y, d_{Y}\right)$ espaços métricos. Se $\left(X, d_{X}\right)$ é fortemente nulo e $f: X \longrightarrow Y$ é uma função lipschitziana então $f[X]$ é fortemente nulo.

Demonstração. Fixe $\left(\varepsilon_{n}\right)_{n \in \omega}$ uma sequência de números reais positivos arbitrária. Como $f: X \longrightarrow Y$ é uma função lipschitziana (Definição 4.1.3) então existe um número real não-negativo $L$ tal que $d_{Y}(f(x), f(y)) \leq L \cdot d_{X}(x, y)$ para cada $x, y \in X$. Temos dois casos para analisar:

$\underline{L=0}$ : Observe que nesse caso $f$ é uma função constante. Logo, $|f[X]|=1$.

$\underline{L>0}$ : Como $X$ é fortemente nulo então temos que existe uma sequência $\left(X_{n}\right)_{n \in \omega}$ de subconjuntos de $X$ tal que $\operatorname{diam}\left(X_{n}\right) \leq \frac{\varepsilon_{n}}{L}$ para cada $n \in \omega$ e $X \subseteq \bigcup_{n \in \omega} X_{n}$. Observe que $f[X] \subseteq \bigcup_{n \in \omega} f\left[X_{n}\right]$. Além disso, para cada $n \in \omega$ temos: 


$$
\begin{aligned}
\operatorname{diam}\left(f\left[X_{n}\right]\right) & \stackrel{4.1 .9}{\leq} L \cdot \operatorname{diam}\left(X_{n}\right) \\
& \stackrel{L>0}{\leq} L \cdot\left(\frac{\varepsilon_{n}}{L}\right) \\
& =\varepsilon_{n} .
\end{aligned}
$$

Teorema 4.1.11 (Carlson [5]). Assuma BC. Seja $(X, d)$ um espaço métrico. Se X é fortemente nulo então X é enumerável.

Demonstração. Pela Proposição 3.1.26 temos que $X$ é separável. Suponha por contradição que $X$ é não-enumerável. Sem perda de generalidade iremos supor que $|X|=\aleph_{1}$.

Iremos demonstrar que vale a desigualdade $|X|<\mathfrak{c}$. Com efeito, $C H \rightarrow \neg B C$ então $B C \rightarrow \neg C H$. Então $\aleph_{1}<\mathfrak{c}$. Por hipótese, temos que $|X|=\aleph_{1}$. Logo, $|X|<\mathfrak{c}$.

Então pelo Lema 4.1 .4 existe $f: X \longrightarrow \mathbb{R}$ injetora e lipschitizana. Como $f$ é lipschitziana e $X$ é fortemente nulo então pela Proposição 4.1 .10 temos que $f[X]$ é fortemente nulo. Como $f$ é injetora então $|f[X]|=\aleph_{1}$. Contradição.

Denotaremos por $B C_{E M}$ a afirmação:

Todo espaço métrico fortemente nulo é enumerável.

Corolário 4.1.12. $B C \longleftrightarrow B C_{E M}$.

Demonstração. ( $\longrightarrow$ ) Pelo Teorema 4.1.11 temos que todo espaço métrico fortemente nulo é enumerável.

$(\longleftarrow)$ Suponha que $X \in \mathcal{S N}$. Como $\mathbb{R}$ é um espaço métrico (métrica euclidiana) então $X$ é um espaço métrico (métrica euclidiana induzida). Por hipótese, temos que $X$ é enumerável. 


\subsection{Propriedade de Rothberger e a Conjectura de Bo- rel em $\mathbb{R}$}

Definição 4.2.1. Denotaremos por $R$ - $B C$ a afirmação:

Se $X \subseteq \mathbb{R}$ possui a propriedade de Rothberger então $X$ é enumerável.

Observação 4.2.2. Se $X \subseteq \mathbb{R}$ é enumerável então $X$ possui a propriedade de Rothberger.

Demonstração. Seja $\left(\mathcal{U}_{n}\right)_{n \in \omega}$ uma sequência de coberturas abertas de $X$. Considere $\left\{x_{n}: n \in \omega\right\}$ uma enumeração de $X$. Fixe $n \in \omega$ arbitrário. Como $\mathcal{U}_{n}$ é uma cobertura de $X$ então existe $U_{n} \in \mathcal{U}_{n}$ tal que $x_{n} \in U_{n}$. Então temos que $X=\left\{x_{n}: n \in \omega\right\} \subseteq$ $\bigcup_{n \in \omega} U_{n}$.

Teorema 4.2.3. Assuma a Conjectura de Borel. Então a afirmação $R$-BC é verdadeira.

Demonstração. Seja $X \subseteq \mathbb{R}$ tal que $X$ possui a propriedade de Rothberger. Então temos que $X$ é fortemente nulo (Proposição 3.1.15). Como vale a Conjectura de Borel então $X$ é enumerável.

Observação 4.2.4. Fixe $\varphi: \mathbb{P} \cap[0,1] \longrightarrow \omega^{\omega}$ homeomorfismo. Seja $F \subseteq[0,1]$ fechado tal que $F \cap \mathbb{Q}=\emptyset$. Como $[0,1]$ é compacto então $F$ é compacto. Logo, $\varphi[F] \subseteq \omega^{\omega} e^{\prime}$ compacto, pois $\varphi$ é continua. Portanto, existe $g \in \omega^{\omega}$ tal que $\varphi[F] \subseteq C_{g}$.

Definição 4.2.5 (Hurewicz, 1927). Seja $(X, d)$ um espaço métrico. Dizemos que $X$ é um espaço de Hurewicz se para cada sequência $\left(\mathcal{G}_{n}\right)_{n \in \omega}$ de coberturas abertas de $X$ existe $\left(\mathcal{F}_{n}\right)_{n \in \omega}$ tal que $\mathcal{F}_{n} \in\left[\mathcal{G}_{n}\right]^{<\omega}$ para cada $n \in \omega$ e $X \subseteq \bigcup_{n<\omega}\left[\bigcap_{m>n} \cup \mathcal{F}_{m}\right]$.

Teorema 4.2.6. Seja $X$ um espaço de Lindelöf tal que $|X|<\mathfrak{b}$. Então $X$ é um espaço de Hurewicz.

Demonstração. Seja $\left(\mathcal{G}_{n}\right)_{n \in \omega}$ uma sequência de coberturas abertas de $X$. Podemos supor que $\left|\mathcal{G}_{n}\right|=\aleph_{0}$ para cada $n \in \omega$, pois $X$ é um espaço de Lindelöf. Fixe $n \in \omega$ arbitrário. Considere $\left\{U_{m}^{n}: m \in \omega\right\}$ uma enumeração sem repetições de $\mathcal{G}_{n}$.

Fixe $x \in X$ qualquer. Defina $f_{x} \in \omega^{\omega}$ como sendo: 


$$
f_{x}(n)=\min \left\{m \in \omega: x \in U_{n}^{m}\right\} .
$$

Como $|X|<\mathfrak{b}$ então $\left|\left\{f_{x} \in \omega^{\omega}: x \in X\right\}\right|<\mathfrak{b}$. Portanto, $\left\{f_{x} \in \omega^{\omega}: x \in X\right\}$ é uma família de funções limitada em $\omega^{\omega}$. Seja $g \in \omega^{\omega}$ tal que $f_{x} \leq^{*} g$ para cada $x \in X$. Defina $\mathcal{F}_{n}=\left\{U_{m}^{n}: m \in g(n)\right\}$ para cada $n \in \omega$. Observe que $\mathcal{F}_{n} \in\left[\mathcal{G}_{n}\right]^{<\omega}$ para cada $n \in \omega$.

Afirmação 4.2.7. O conjunto $\left\{n \in \omega: x \in \bigcup \mathcal{F}_{n}\right\}$ é cofinito em $\omega$ para cada $x \in X$.

Demonstração. Fixe $x \in X$ arbitrário. Note que:

$$
\begin{aligned}
\left|\left\{n \in \omega: x \notin \bigcup \mathcal{F}_{n}\right\}\right| & =\left|\left\{n \in \omega: x \notin \bigcup_{m \in g(n)} U_{n}^{m}\right\}\right| \\
& =\left|\left\{n \in \omega: f_{x}(n)>g(n)\right\}\right| \\
& <\omega .
\end{aligned}
$$

Por definição, temos que $X$ é um espaço de Hurewicz.

Proposição 4.2.8. Se $X \subseteq 2^{\omega}$ e $|X|<\mathfrak{b}$ então $X$ é um espaço de Hurewicz.

Demonstração. Como $2^{\omega}$ é um espaço compacto então $2^{\omega}$ é um espaço de Lindelöf. Então $2^{\omega}$ possui base enumerável. Portanto, $X \subseteq 2^{\omega}$ é um espaço métrico (munido da métrica induzida) que possui base enumerável. Logo, $X$ é um espaço de Lindelöf. Como $|X|<\mathfrak{b}$ então pelo Teorema 4.2 .6 temos que $X$ é um espaço de Hurewicz.

Teorema 4.2.9. Seja $(X, d)$ um espaço métrico. Se $X$ é um espaço de Hurewicz e $X$ é fortemente nulo então $X$ é um espaço de Rothberger.

Demonstração. Seja $\left(\mathcal{G}_{n}\right)_{n \in \omega}$ uma sequência de coberturas abertas de $X$. Considere $n<\omega$ arbitrário. Defina:

$$
\mathcal{H}_{n}=\left\{B_{\varepsilon}(x): x \in X, \varepsilon>0 \text { e } \exists G \in \mathcal{G}_{n}\left(B_{3 \varepsilon}(x) \subseteq G\right)\right\} .
$$

Afirmação 4.2.10. $\mathcal{H}_{n}$ é uma cobertura por abertos de $X$. 
Demonstração. Fixe $x \in X$ arbitrário. Como $\mathcal{G}_{n}$ é uma cobertura por abertos para $X$ então existe $G \in \mathcal{G}_{n}$ tal que $x \in G$. Seja $\delta>0$ tal que $x \in B_{\delta}(x) \subseteq G$. Tome $\varepsilon=\frac{\delta}{3}$. Logo, temos que $x \in B_{3 \varepsilon}(x)=B_{\delta}(x) \subseteq G$. Então $B_{\delta}(x) \in \mathcal{H}_{n}$.

Como $X$ é um espaço de Hurewicz então existe $\left(\mathcal{F}_{n}\right)_{n \in \omega}$ tal que $\mathcal{F}_{n} \in\left[\mathcal{H}_{n}\right]^{<\omega}$ para cada $n \in \omega$ e $X \subseteq \bigcup_{n<\omega}\left[\bigcap_{m>n} \cup \mathcal{F}_{m}\right]$.

Fixe $n<\omega$. Vamos supor que $\mathcal{F}_{n} \neq \emptyset$. Escreva $\mathcal{F}_{n}=\left\{B_{\varepsilon_{i}^{n}}\left(x_{i}^{n}\right): i \in I_{n}\right\}$ sendo que $I_{n} \subseteq \omega$ é finito. Defina $\varepsilon_{n}=\min \left\{\varepsilon_{i}^{n}: i \in I_{n}\right\}$. Como $\mathcal{F}_{n} \neq \emptyset$ então $\varepsilon_{n}>0$. Considere $\left(J_{k}\right)_{k \in \omega}$ uma partição de $\omega$ sendo que $\left|J_{k}\right|=\aleph_{0}$ para cada $k<\omega$.

Como $X$ é um espaço métrico fortemente nulo então decorre da definição de espaço de Hurewicz que temos que para cada $k<\omega$ existe $\left(y_{n}\right)_{n \in J_{k}}$ tal que $X=\bigcup_{n \in J_{k}} B_{\varepsilon_{n}}\left(y_{n}\right)$. Fixe $n<\omega$ arbitrário. Escolha $G_{n} \in \mathcal{G}_{n}$ tal que $B_{\varepsilon_{n}}\left(y_{n}\right) \subseteq G_{n}$; se tal escolha não for possível então tome $G_{n} \in \mathcal{G}_{n}$ arbitrário.

Considere $x \in X$. Como $X \subseteq \bigcup_{n<\omega}\left[\bigcap_{m>n} \cup \mathcal{F}_{m}\right]$ então existe $m<\omega$ tal que $x \in \bigcap_{n \geq m} \cup \mathcal{F}_{n}$. Seja $k<\omega$ tal que $n>m$ para cada $n \in J_{k}$. Como $X=\bigcup_{n \in J_{k}} B_{\varepsilon_{n}}\left(y_{n}\right)$ então existe $n \in J_{k}$ tal que $x \in B_{\varepsilon_{n}}\left(y_{n}\right)$. Como $n>m$ e $x \in \bigcap_{n \geq m} \cup \mathcal{F}_{n}$ então existe $i \in I_{n}$ tal que $x \in B_{\varepsilon_{i}^{n}}\left(x_{i}^{n}\right)$ (aqui estamos usando a definição de $\mathcal{F}_{n}$ ).

Afirmação 4.2.11. Vale a inclusão $B_{\varepsilon_{n}}\left(y_{n}\right) \subseteq B_{3 \varepsilon_{i}^{n}}\left(x_{i}^{n}\right)$.

Demonstração. Fixe $z \in B_{\varepsilon_{n}}\left(y_{n}\right)$ arbitrário. Aplicando a desigualdade triangular temos que:

$$
\begin{aligned}
d\left(z, x_{i}^{n}\right) & \leq d\left(z, y_{n}\right)+d\left(y_{n}, x\right)+d\left(x, x_{i}^{n}\right) \\
& <\varepsilon_{n}+\varepsilon_{n}+\varepsilon_{i}^{n} \\
& \leq \varepsilon_{i}^{n}+\varepsilon_{i}^{n}+\varepsilon_{i}^{n} \\
& =3 \varepsilon_{i}^{n} .
\end{aligned}
$$

Logo, $z \in B_{3 \varepsilon_{i}^{n}}\left(x_{i}^{n}\right)$.

Pela definição de $\mathcal{H}_{n}$ temos que existe $G \in \mathcal{G}_{n}$ tal que $B_{3 \varepsilon_{i}^{n}}\left(x_{i}^{n}\right) \subseteq G$. Como $B_{\varepsilon_{n}}\left(y_{n}\right) \subseteq B_{3 \varepsilon_{i}^{n}}\left(x_{i}^{n}\right)$ e $B_{3 \varepsilon_{i}^{n}}\left(x_{i}^{n}\right) \subseteq G$ então $B_{\varepsilon_{n}}\left(y_{n}\right) \subseteq G$. Logo, $B_{\varepsilon_{n}}\left(y_{n}\right) \subseteq G_{n}$ e $x \in G_{n}$. Portanto, $X \subseteq \bigcup_{n<\omega} G_{n}$.

Corolário 4.2.12. Se a afirmação R-BC é verdadeira então vale a Conjectura de Borel. 
Demonstração. Sabemos que $\omega_{1} \leq \mathfrak{b}$. Se $\omega_{1}=\mathfrak{b}$ então o resultado decorre do Lema 2.2 .21 e da Proposição 3.1.16.

Suponha que $\omega_{1}<\mathfrak{b}$ e que $X \subseteq 2^{\omega}$ é fortemente nulo. Podemos supor que $|X|=\omega_{1}$. Como $X$ é um espaço de Hurewicz (Proposição 4.2.8) então pelo Teorema 4.2 .9 temos que $X$ possui a propriedade de Rothberger.

Seja $\psi: \omega^{\omega} \longrightarrow[0,1] \cap \mathbb{P}$ um homeomorfismo. Como a propriedade de Rothberger é uma propriedade topológica então $\psi[X] \subseteq[0,1]$ possui a propriedade de Rothberger. Como $X$ é não enumerável e $\psi$ é um homeomorfismo então $\psi[X]$ é não enumerável.

Corolário 4.2.13. A Conjectura de Borel é equivalente a afirmação $R-B C$. 


\subsection{Jogos}

Definição 4.3.1. Seja $(X, d)$ um espaço métrico. Denotaremos por $G(X)$ o jogo de $\omega$ rodadas entre os jogadores $I$ e $I I$ definido da seguinte forma:

- Fixe $n \in \omega$. Na $n$-ésima rodada o jogador $I$ escolhe um número real $\varepsilon_{n}>0$ e o jogador $I I$ responde escolhendo $B_{n} \subseteq X$ tal que $\operatorname{diam}\left(B_{n}\right) \leq \varepsilon_{n}$. O jogador $I I$ vence a partida $\left(\varepsilon_{0}, B_{0}, \ldots, \varepsilon_{n}, B_{n}, \ldots\right)$ do jogo $G(X)$ se $\bigcup_{n \in \omega} B_{n}=X$; caso contrário o jogador $I$ vence.

Definição 4.3.2. Seja $(X, d)$ um espaço métrico. Dizemos que $\sigma:{ }^{<\omega} \mathbb{R}_{>0} \backslash\{\emptyset\} \longrightarrow$ $\wp(X)$ é uma estratégia vencedora para o jogador $I I$ em $G(X)$ se $\bigcup_{n \in \omega} \sigma\left(\varepsilon_{0}, \ldots, \varepsilon_{n}\right)=X$ para cada sequência de números reais positivos $\left(\varepsilon_{n}\right)_{n \in \omega}$.

Teorema 4.3.3. Seja $(X, d)$ um espaço métrico. Então o jogador II possui estratégia vencedora em $G(X)$ se, e somente se, $X$ é enumerável.

Demonstração. Suponha que $X$ é enumerável. Seja $\left\{x_{n}: n \in \omega\right\}$ uma enumeração para $X$. Observe que $\sigma:{ }^{<\omega} \mathbb{R}_{>0} \backslash\{\emptyset\} \longrightarrow \wp(X)$ dada por $\sigma\left(\varepsilon_{0}, \ldots, \varepsilon_{n}\right)=\left\{x_{n}\right\}$ para cada sequência finita $\left(\varepsilon_{0}, \ldots, \varepsilon_{n}\right) \in{ }^{<\omega} \mathbb{R}_{>0}$ é uma estratégia vencedora para o jogador $I I$.

Seja $\sigma:{ }^{<\omega} \mathbb{R}_{>0} \backslash\{\emptyset\} \longrightarrow \wp(X)$ estratégia vencedora para o jogador $I I$, isto é, para cada sequência de números reais positivos $\left(\varepsilon_{n}\right)_{n \in \omega}$ temos que $\bigcup_{n \in \omega} \sigma\left(\varepsilon_{0}, \ldots, \varepsilon_{n}\right)=X$.

Fixe $x \in X$ arbitrário. Temos a seguinte:

Afirmação 4.3.4. Existe uma sequência finita de números racionais positivos $s(x)=$ $\left(r_{0}^{x}, \ldots, r_{n(x)}^{x}\right)$ tal que $x \in \sigma\left(r_{0}^{x}, \ldots, r_{n(x)}^{x}, r\right)$ para cada número racional $r>0$.

Demonstração. Suponha por absurdo que para cada sequência finita de números racionais positivos $\left(r_{0}^{x}, \ldots, r_{n}^{x}\right)$ existe um número racional $r_{n+1}^{x}>0$ tal que $x \notin \sigma\left(r_{0}^{x}, \ldots, r_{n}^{x}, r_{n+1}^{x}\right)$. Considere a partida $\left(r_{0}^{x}, \sigma\left(r_{0}^{x}\right), \ldots, r_{n}^{x}, \sigma\left(r_{0}^{x}, \ldots, r_{n}^{x}\right), \ldots\right)$ do jogo $G(X)$. Observe que $x \notin$ $\bigcup_{n \in \omega} \sigma\left(r_{0}^{x}, \ldots, r_{n}^{x}\right)$. Portanto, $\bigcup_{n \in \omega} \sigma\left(r_{0}^{x}, \ldots, r_{n}^{x}\right) \neq X$. Então o jogador $I I$ não vence a partida $\left(r_{0}^{x}, \sigma\left(r_{0}^{x}\right), \ldots, r_{n}^{x}, \sigma\left(r_{0}^{x}, \ldots, r_{n}^{x}\right), \ldots\right)$. Contradição, pois $\sigma$ é uma estratégia vencedora.

Considere $s: X \longrightarrow<\omega \mathbb{Q}_{>0}$ dada por $s(x)=\left(r_{1}^{x}, \ldots, r_{n(x)}^{x}\right)$ para cada $x \in X$.

Afirmação 4.3.5. s é uma função injetora. 
Demonstração. Sejam $x, y \in X$ tais que $s(x)=s(y)$. Pela definição de $s$ temos que $\sigma\left(r_{0}^{x}, \ldots, r_{n(x)}^{x}\right)=\sigma\left(r_{0}^{y}, \ldots, r_{n(y)}^{y}\right)$. Pela forma como $\sigma\left(r_{0}^{x}, \ldots, r_{n(x)}^{x}\right)$ e $\sigma\left(r_{0}^{y}, \ldots, r_{n(y)}^{y}\right)$ foram escolhidos temos que $x, y \in \sigma\left(r_{0}^{x}, \ldots, r_{n(x)}^{x}, r\right)$ para cada número racional $r>0$. Em particular, $x, y \in \sigma\left(r_{0}^{x}, \ldots, r_{n(x)}^{x}, \frac{1}{k+1}\right)$ para cada $k \in \omega$. Defina $B_{k}=\sigma\left(r_{0}^{x}, \ldots, r_{n(x)}^{x}, \frac{1}{k+1}\right)$ para cada $k \in \omega$. Como $x, y \in B_{k} \mathrm{e} \operatorname{diam}\left(B_{k}\right) \leq \frac{1}{k+1}$ para cada $k \in \omega$ então $d(x, y) \leq$ $\frac{1}{k+1}$ para cada $k \in \omega$. Portanto, $x=y$.

Então temos que $|X| \leq\left.\right|^{<\omega} \mathbb{Q}_{>0} \mid=\aleph_{0}$. Portanto, $X$ é enumerável.

Definição 4.3.6. Seja $(X, d)$ um espaço métrico. Dizemos que $X$ é totalmente limitado se para cada $\varepsilon>0$ existem $x_{0}, \ldots, x_{n} \in X$ tais que $X=\bigcup_{i=0}^{n} B_{\varepsilon}\left(x_{i}\right)$. E dizemos que $X$ é $\sigma$-totalmente limitado se existe $\left(X_{n}\right)_{n \in \omega}$ tal que $X_{n} \subseteq X$ é totalmente limitado para cada $n \in \omega$ e $X=\bigcup_{n \in \omega} X_{n}$.

Definição 4.3.7. Sejam $(X, d)$ um espaço métrico $\sigma$-totalmente limitado e $\mathcal{F}=\left(F_{n}\right)_{n \in \omega}$ tal que $F_{n} \subseteq X$ é totalmente limitado para cada $n \in \omega, F_{n} \subseteq F_{n+1}$ para cada $n \in \omega$ e $X=\bigcup_{n \in \omega} F_{n}$. Denotaremos por $G(X, \mathcal{F})$ o jogo de $\omega$ rodadas entre os jogadores $I$ e II definido da seguinte forma:

- Fixe $n \in \omega$. Na $n$-ésima rodada o jogador $I$ escolhe um número real $\varepsilon_{n}>0$ e o jogador $I I$ responde escolhendo $B_{n} \subseteq F_{n}$ tal que $\operatorname{diam}\left(B_{n}\right) \leq \varepsilon_{n}$. O jogador $I I$ vence a partida $\left(\varepsilon_{0}, B_{0}, \ldots, \varepsilon_{n}, B_{n}, \ldots\right)$ do jogo $G(X, \mathcal{F})$ se $\bigcap_{n \in \omega} \bigcup_{m \geq n} B_{m}=X$; caso contrário o jogador $I$ vence.

Lema 4.3.8. Sejam $(X, d)$ um espaço métrico totalmente limitado e $\delta>0$. Então existe $\emptyset \neq \mathcal{B} \subseteq \wp(X)$ tal que $|\mathcal{B}|<\aleph_{0}$, $\operatorname{diam}(B) \leq \delta$ para cada $B \in \mathcal{B}$ e para cada $Y \subseteq X$ com $\operatorname{diam}(Y) \leq \frac{1}{3} \delta$ existe $B \in \mathcal{B}$ tal que $Y \subseteq B$.

Demonstração. Como $X$ é um espaço métrico totalmente limitado então existem $X_{0}, \ldots, X_{m} \subseteq$ $X$ tais que $\operatorname{diam}\left(X_{j}\right) \leq \frac{\delta}{3}$ para cada $j=0, \ldots, m$ e $X=\bigcup_{j=0}^{m} X_{j}$. Defina $W_{j}=$ $\bigcup_{u \in X_{j}} B_{\frac{\delta}{3}}[u]$ para cada $j=0, \ldots, m$. Vamos mostrar que $\mathcal{B}=\left\{W_{0}, \ldots, W_{m}\right\}$ satisfaz as propriedades desejadas.

Afirmação 4.3.9. $\operatorname{diam}\left(X_{j}\right) \leq \delta$ para cada $j=0, \ldots, m$.

Demonstração. Fixe $j \in\{0, \ldots, m\}$ arbitrário. Se $x, y \in W_{j}$ então existem $u, u^{\prime} \in W_{j}$ tais que $x \in B_{\frac{\delta}{3}}[u]$ e $y \in B_{\frac{\delta}{3}}\left[u^{\prime}\right]$. Usando a desigualdade triangular temos que: 


$$
\begin{aligned}
d(x, y) & \leq d(x, u)+d\left(u, u^{\prime}\right)+d\left(u^{\prime}, y\right) \\
& \leq \frac{\delta}{3}+\frac{\delta}{3}+\frac{\delta}{3} \\
& =\delta
\end{aligned}
$$

Afirmação 4.3.10. Se $\emptyset \neq Y \subseteq X$ e $\operatorname{diam}(Y) \leq \frac{\delta}{3}$ então existe $j \in\{0, \ldots, m\}$ tal que $Y \subseteq W_{j}$

Demonstração. Como $X=\bigcup_{j=0}^{m} X_{j}$ então existe $j \in\{0, \ldots, m\}$ tal que $X_{j} \cap Y \neq \emptyset$. Fixe $x \in X_{j} \cap Y$. Considere $y \in Y$ arbitrário. Como $x \in Y$ e $\operatorname{diam}(Y) \leq \frac{\delta}{3}$ então $y \in B_{\frac{\delta}{3}}[x]$. Como $x \in X_{j}$ então temos que:

$$
y \in B_{\frac{\delta}{3}}[x] \subseteq \bigcup_{u \in X_{j}} B_{\frac{\delta}{3}}[u]=W_{j}
$$

Lema 4.3.11. Se X é fortemente nulo então o jogador I não possui estratégia vencedora em $G(X, \mathcal{F})$.

Demonstração. Suponha que $\sigma:<\omega \wp(X) \longrightarrow \mathbb{R}_{>0}$ é uma estratégia vencedora para o jogador $I$ no jogo $G(X, \mathcal{F})$. Por aplicações sucessivas do Lema 4.3 .8 podemos obter recursivamente $\delta_{n}, \mathcal{B}_{n}$ para cada $n \in \omega$ tais que:

(i) $\delta_{n}=\min \left\{\sigma\left(B_{0}, \ldots, B_{n-1}\right): B_{0} \in \mathcal{B}_{0}, \ldots, B_{n-1} \in \mathcal{B}_{n-1}\right\}$ para cada $n \in \omega$;

(ii) $\emptyset \neq \mathcal{B}_{n} \subseteq \wp\left(F_{n}\right)$ tal que $\left|\mathcal{B}_{n}\right|<\aleph_{0}$ para cada $n \in \omega$ e $\operatorname{diam}(B) \leq \delta_{n}$ para cada $B \in \mathcal{B}_{n}$ e $n \in \omega$

(iii) para cada $Y_{n} \subseteq F_{n} \operatorname{com} \operatorname{diam}\left(Y_{n}\right) \leq \frac{1}{3} \delta_{n}$ existe $B_{n} \in \mathcal{B}_{n}$ tal que $Y_{n} \subseteq B_{n}$.

Seja $\left(M_{n}\right)_{n \in \omega}$ como no Lema 2.2.14. Defina $\alpha_{k}=\frac{1}{3} \delta_{k}$ para cada $k \in \omega$. Como $X$ é fortemente nulo então $F_{n} \subseteq X$ é fortemente nulo para cada $n \in \omega$. Seja $\left(A_{k}\right)_{k \in M_{n}}$ tal que $\operatorname{diam}\left(A_{k}\right) \leq \alpha_{k}$ para cada $k \in M_{n}$ e $F_{n} \subseteq \bigcup_{k \in M_{n}} A_{k}$. 
Se $k \in \omega$ então existe $n \in \omega$ tal que $k \in M_{n}$, pois $\left(M_{n}\right)_{n \in \omega}$ é uma partição de $\omega$. Como $\operatorname{diam}\left(A_{k}\right) \leq \alpha_{k}$ então existe $B_{k} \in \mathcal{B}_{k}$ tal que $A_{k} \subseteq B_{k}$ (condição (iii)). Defina $\varepsilon_{n}=\sigma\left(B_{0}, \ldots, B_{n-1}\right)$ para cada $n \in \omega$. Como $B_{n} \subseteq F_{n}$ (condição $\left.(i i)\right)$ e $\operatorname{diam}\left(B_{n}\right) \leq$ $\delta_{n} \leq \varepsilon_{n}$ (condições $(i)$ e $\left.(i i)\right)$ para cada $n \in \omega$ então $\left(\varepsilon_{0}, B_{0}, \ldots, \varepsilon_{n}, B_{n}, \ldots\right)$ é uma partida do jogo $G(X, \mathcal{F})$.

Resta mostrarmos que $X \subseteq \bigcap_{n \in \omega} \bigcup_{m \geq n} B_{m}$. Sejam $x \in X$ e $n \in \omega$. Devemos demonstrar que $x \in \bigcup_{m \geq n} B_{m}$. Como $X=\bigcup_{n \in \omega} F_{n}$ então existe $j \in \omega$ tal que $x \in F_{j}$. Como $F_{j} \subseteq F_{j+1}$ para cada $j \in \omega$ então $x \in F_{i}$ para cada $i \geq j$. Fixe $i \geq j$. Como $F_{i} \subseteq \bigcup_{k \in M_{i}} A_{k}$ então existe $k \in \omega$ tal que $x \in A_{k} \subseteq B_{k}$. Como $\left(M_{n}\right)_{n \in \omega}$ é uma partição de $\omega$ então $M_{i} \cap M_{j}=\emptyset$ para cada $i, j \in \omega$ tal que $i \neq j$. Então $\left|\left\{k \in \omega: x \in B_{k}\right\}\right| \geq \aleph_{0}$. Escolha $m \in\left\{k \in \omega: x \in B_{k}\right\}$ tal que $m \geq n$ (podemos fazer tal escolha, pois $\left|\left\{k \in \omega: x \in B_{k}\right\}\right| \geq \aleph_{0}$ ). Então $x \in B_{m} \subseteq \bigcup_{m \geq n} B_{m}$. Como queríamos.

Teorema 4.3.12. Sejam $(X, d)$ um espaço métrico $\sigma$-totalmente limitado e $\mathcal{F}=\left(F_{n}\right)_{n \in \omega}$ tal que $F_{n} \subseteq X$ é totalmente limitado para cada $n \in \omega, F_{n} \subseteq F_{n+1}$ para cada $n \in \omega e$ $X=\bigcup_{n \in \omega} F_{n}$. As seguintes afirmações são equivalentes:

(1) X não é fortemente nulo;

(2) o jogador I possui estratégia vencedora em $G(X)$;

(3) o jogador I possui estratégia vencedora em $G(X, \mathcal{F})$.

Demonstração. Iremos mostrar que (1) $\Rightarrow(2),(2) \Rightarrow(3)$ e $\neg(1) \Rightarrow \neg(3)$.

$(1) \Rightarrow(2)$ : Suponha que $X$ não é um espaço fortemente nulo. Então existe uma sequência de números reais positivos $\left(\varepsilon_{n}\right)_{n \in \omega}$ tal que para cada $\left(X_{n}\right)_{n \in \omega} \operatorname{com} \operatorname{diam}\left(X_{n}\right) \leq$ $\varepsilon_{n}$ temos que $\bigcup_{n \in \omega} X_{n} \neq X$.

Considere a estratégia $\sigma:{ }^{<\omega} \wp(X) \longrightarrow \mathbb{R}_{>0}$ para o jogador $I$ dada por $\sigma(\emptyset)=\varepsilon_{0}$ e $\sigma\left(B_{0}, \ldots, B_{n-1}\right)=\varepsilon_{n}$ para cada $n \geq 1$. Vamos mostrar que $\sigma$ é uma estratégia vencedora. Fixe $\left(B_{n}\right)_{n \in \omega} \in{ }^{\omega}{ }_{\wp}(X)$ arbitrária. Considere a partida $\left(\varepsilon_{0}, B_{0}, \ldots \varepsilon_{n}, B_{n}, \ldots\right)$ do jogo $G(X)$. Como $\operatorname{diam}\left(B_{n}\right) \leq \varepsilon_{n}$ para cada $n \in \omega$ então $\bigcup_{n \in \omega} B_{n} \neq X$. Logo, o jogador $I$ vence a partida $\left(\varepsilon_{0}, B_{0}, \ldots, \varepsilon_{n}, B_{n}, \ldots\right)$.

$(2) \Rightarrow(3)$ : Considere $\sigma:{ }^{<\omega} \wp(X) \longrightarrow \mathbb{R}_{>0}$ estratégia vencedora para o jogador $I \mathrm{em}$ $G(X)$. Seja $\left(B_{n}\right)_{n \in \omega} \in{ }^{\omega} \wp(X)$ tal que $B_{n} \subseteq F_{n}$ para cada $n \in \omega$. Defina $\varepsilon_{0}=\sigma(\emptyset)$ e $\varepsilon_{n}=\sigma\left(B_{0}, \ldots, B_{n-1}\right)$ para cada $n \geq 1$. Considere a partida $\left(\varepsilon_{0}, B_{0}, \ldots, \varepsilon_{n}, B_{n}, \ldots\right)$ do 
jogo $G(X)$. Como $\sigma$ é uma estratégia vencedora então $\bigcup_{n \in \omega} B_{n} \neq X$. Observe que $\bigcap_{m \in \omega} \bigcup_{n \geq m} B_{n} \neq X$.

Portanto, $\sigma$ é uma estratégia vencedora para o jogador $I$ em $G(X, \mathcal{F})$.

$\neg(1) \Rightarrow \neg(3)$ : Decorre imediatamente do Lema 4.3.11.

Provamos (Teorema 4.3.3) que o jogador $I I$ possui estratégia vencedora em $G(X)$ se e somente se $X$ for enumerável. E mostramos (Teorema 4.3.12) que $X$ é fortemente nulo se e somente se o jogador $I$ não possui estratégia vencedora no jogo $G(X)$. Iremos extrair um corolário que nos dá uma conecção entre a Conjectura de Borel e o jogo $G(X)$. Para isso precisamos da seguinte:

Definição 4.3.13. Dizemos que o jogo $G(X)$ é determinado se algum dos jogadores possui uma estratégia vencedora.

Observe que a definição acima diz que se o jogador $I$ não possui estratégia vencedora em $G(X)$ então o jogador $I I$ possui estratégia vencedora em $G(X)$ e se o jogador $I I$ não possui estratégia vencedora em $G(X)$ então o jogador $I$ possui estratégia vencedora em $G(X)$.

Com isso temos o seguinte corolário que nos dá a conexão desejada:

Corolário 4.3.14. Vale a Conjectura de Borel se e somente se para cada $X \subseteq \mathbb{R}$ temos que o jogo $G(X)$ é determinado.

Demonstração. $(\leftarrow)$ Seja $Y \subseteq \mathbb{R}$ um conjunto fortemente nulo. Pelo Teorema 4.3.12 temos que o jogador $I$ não possui estratégia vencedora em $G(Y)$. Por hipótese $G(X)$ é um jogo determinado para cada $X \subseteq \mathbb{R}$. Em particular $G(Y)$ é um jogo determinado. Como o jogador $I$ não possui estratégia vencedora em $G(Y)$ e $G(Y)$ é um jogo determinado então o jogador $I I$ possui uma estratégia vencedora em $G(Y)$. Pelo Teorema 4.3 .3 temos que $Y$ é um conjunto enumerável. Como $Y \in \mathcal{S N}$ foi escolhido de forma arbitrária então vale a Conjectura de Borel.

$(\rightarrow)$ Fixe $X \subseteq \mathbb{R}$ arbitrário. Iremos analisar dois casos:

$X \in \mathcal{S N}$ : Por hipótese vale a Conjectura de Borel. Então $X$ é um conjunto enumerável. Pelo Teorema 4.3.3 temos que o jogador $I I$ possui uma estratégia vencedora em $G(X)$. Logo, $G(X)$ é um jogo determinado.

$X \notin \mathcal{S N}$ : Pelo Teorema 4.3 .12 temos que o jogador $I$ possui uma estratégia vencedora em $G(X)$. Portanto, $G(X)$ é um jogo determinado. 


\section{Capítulo 5}

\section{Espaços Fortemente Magros}

\subsection{Teorema de Erdôs-Sierpiński}

O objetivo dessa seção é apresentar o Teorema de Erdôs-Sierpiński. Esse resultado nos mostra que assumindo a hipótese $\operatorname{add}(\mathcal{M})=\operatorname{add}(\mathcal{N})=\mathfrak{c}$ temos uma certa dualidade entre os subconjuntos de medida zero da reta real e os subconjuntos magros da reta real. Tal dualidade é expressa por uma função bijetora $f: \mathbb{R} \longrightarrow \mathbb{R}$ que é construída usando-se a técnica de indução transfinita.

Adicionalmente, apresentaremos duas formas de relacionar conjuntos de Luzin e conjuntos de Sierpiński. A primeira maneira apresentada por Rothberger [13] e a segunda através da função dada pelo Teorema de Erdôs-Sierpiński.

De maneira análoga ao conjunto de Luzin temos o conjunto de Sierpiński.

Definição 5.1.1. Seja $S \subseteq \mathbb{R}$. Dizemos que $S$ é um conjunto de Sierpiński se $S$ é não-enumerável e para cada $N \subseteq \mathbb{R}$ tal que $N$ possui medida de Lebesgue igual a zero temos que $S \cap N$ é enumerável.

Assumindo a Hipótese do Contínuo podemos demonstrar a existência de um conjunto de Sierpiński de forma análoga que demonstramos a existência de um conjunto de Luzin.

Teorema 5.1.2 (CH). Existe $S \subseteq \mathbb{R}$ conjunto de Sierpiński.

O resultado a seguir nos mostra que existe uma partição da reta real em um conjunto nulo e um conjunto magro. 
Teorema 5.1.3. Existem $A, B \subseteq \mathbb{R}$ disjuntos tais que $A \in \mathcal{N}, B \in \mathcal{M}$ e $A \cup B=\mathbb{R}$.

Demonstração. Seja $\left\{q_{n}: n \in \omega\right\}$ uma enumeração sem repetições de $\mathbb{Q}$. Defina $U_{m}=$ $\left.\bigcup_{n>m}\right] q_{n}-\frac{1}{2^{n}}, q_{n}+\frac{1}{2^{n}}\left[\right.$ para cada $m \in \omega$. Observe que $U_{m}$ é um aberto denso de $\mathbb{R}$.

Afirmação 5.1.4. $\mu\left(U_{m}\right) \leq 2^{1-m}$ para cada $m \in \omega$.

Demonstração. Fixe $m \in \omega$ arbitrário. Observe que:

$$
\begin{aligned}
\mu\left(U_{m}\right) & =\mu\left(\bigcup_{n>m}\right] q_{n}-\frac{1}{2^{n}}, q_{n}+\frac{1}{2^{n}}[) \\
& \leq \sum_{n>m} \mu(] q_{n}-\frac{1}{2^{n}}, q_{n}+\frac{1}{2^{n}}[) \\
& =\sum_{n>m}\left[\left(q_{n}+\frac{1}{2^{n}}\right)-\left(q_{n}-\frac{1}{2^{n}}\right)\right] \\
& =\sum_{n>m} 2 \cdot \frac{1}{2^{n}} \\
& =\sum_{n>m} \frac{1}{2^{n-1}} \\
& =2^{1-m} .
\end{aligned}
$$

Defina $A=\bigcap_{m \in \omega} U_{m}$. Observe que $U_{m} \subseteq U_{n}$ para cada $m, n \in \omega$ tais que $n \leq m$. Logo, temos que:

$$
\mu(A)=\mu\left(\bigcap_{m \in \omega} U_{m}\right)=\lim _{m \in \omega} 2^{1-m}=0 .
$$

Considere $B=\mathbb{R} \backslash A$. Como $U_{m}$ é um aberto denso de $\mathbb{R}$ para cada $m \in \omega$ então $A=\bigcap_{m \in \omega} U_{m}$ é um $G_{\delta}$ denso em $\mathbb{R}$ (Teorema de Baire). Logo, temos que $B \in \mathcal{M}$.

A seguir apresentamos, que com a hipótese adicional de não enumerabilidade, subconjuntos de um conjunto de Luzin também são conjuntos de Luzin.

Lema 5.1.5. Seja $X$ um conjunto de Luzin. Se $Y \subseteq X$ e $|Y|>\aleph_{0}$ então $Y$ é um conjunto de Luzin. 
Demonstração. Suponha por absurdo que $Y$ não é um conjunto de Luzin. Então $|Y| \leq$ $\aleph_{0}$ ou existe $M_{0} \in \mathcal{M}$ tal que $\left|Y \cap M_{0}\right|>\aleph_{0}$. Por hipótese, $|Y| \leq \aleph_{0}$ não ocorre. Logo, $\left|Y \cap M_{0}\right|>\aleph_{0}$ para algum $M_{0} \in \mathcal{M}$. Como $Y \subseteq X$ então temos que:

$$
\aleph_{0}<\left|Y \cap M_{0}\right| \leq\left|X \cap M_{0}\right|
$$

Contradição, pois $X$ é um conjunto de Luzin.

De maneira análoga à demonstração do Lema 5.1 .5 podemos demonstrar o seguinte lema:

Lema 5.1.6. Seja $X \subseteq \mathbb{R}$ um conjunto de Sierpiński. Se $Y \subseteq X$ e $|Y|>\aleph_{0}$ então $Y$ é um conjunto de Sierpiński.

Lema 5.1.7. Se $X$ é um conjunto de Luzin então $X \notin \mathcal{M}$.

Demonstração. Suponha por absurdo que existe $X \subseteq \mathbb{R}$ tal que $X$ é um conjunto de Luzin e $X \in \mathcal{M}$. Como $X$ é um conjunto de Luzin então $|X|>\aleph_{0}$. Logo, existe $Y \subseteq X$ tal que $|Y|=\aleph_{1}$. Como $X \in \mathcal{M}$ e $\mathcal{M}$ é um ideal então $Y \in \mathcal{M}$. Note que:

$$
|X \cap Y|=|Y|=\aleph_{1} .
$$

Contradição, pois como $X$ é um conjunto de Luzin deveríamos ter $|X \cap M| \leq \aleph_{0}$ para cada $M \in \mathcal{M}$.

De forma análoga a demonstração do Lema 5.1 .7 também podemos demonstrar o resultado abaixo:

Lema 5.1.8. Se $X$ é um conjunto de Sierpiński então $X \notin \mathcal{N}$.

O Teorema 5.1.9 nos dá uma cobertura de cardinalidade $\kappa\left(\aleph_{0}<\kappa \leq \mathfrak{c}\right)$ para a reta real constítuida por conjuntos de medida zero.

Teorema 5.1.9. Seja $\kappa \leq \mathfrak{c}$ um número cardinal. Suponha que existe $X \in[\mathbb{R}]^{\kappa}$ tal que $X \notin \mathcal{M}$. Então existe $\left(Y_{\xi}\right)_{\xi<\kappa}$ tal que $Y_{\xi} \in \mathcal{N}$ para cada $\xi<\kappa$ e $\mathbb{R}=\bigcup_{\xi<\kappa} Y_{\xi}$.

Demonstração. Considere $A$ dado pelo Lema 5.1.3. Seja $\left\{x_{\xi}: \xi<\kappa\right\}$ uma indexação sem repetições de $X$. Defina $Y_{\xi}=A+x_{\xi}$ para cada $\xi<\kappa$. Note que $Y_{\xi} \in \mathcal{N}$ para cada $\xi<\kappa$, pois $A \in \mathcal{N}$. 
Demonstraremos que $\mathbb{R}=\bigcup_{\xi<\kappa} Y_{\xi}$. De fato, suponha por absurdo que $\mathbb{R} \neq \bigcup_{\xi<\kappa} Y_{\xi}$. Então existe $z \in \mathbb{R} \backslash \bigcup_{\xi<\kappa} Y_{\xi}$. Portanto, $z \notin Y_{\xi}$ para cada $\xi<\kappa$. Como $Y_{\xi}=A+x_{\xi}$ para cada $\xi<\kappa$ então $z \notin A+x_{\xi}$ para cada $\xi<\kappa$. Logo, $(z-A) \cap\left\{x_{\xi}: \xi<\kappa\right\}=\emptyset$. De fato, suponha por absurdo que existe $w \in(z-A) \cap\left\{x_{\xi}: \xi<\kappa\right\}$. Então $w=x_{\gamma}$ para algum $\gamma<\kappa$ e $w=z-a$ para algum $a \in A$. Portanto, $z=a+x_{\gamma}$. Contradição, pois $z \notin A+x_{\xi}$ para cada $\xi<\kappa$.

Como $(z-A) \cap\left\{x_{\xi}: \xi<\kappa\right\}=\emptyset$ então $X \subseteq(\mathbb{R} \backslash z-A) \in \mathcal{M}$. Logo, $X \in \mathcal{M}$ Contradição.

O resultado análogo ao Teorema 5.1.9 é dado abaixo e a sua demonstração é também análoga à do Teorema 5.1.9.

Teorema 5.1.10. Seja $\kappa \leq \mathfrak{c}$ um número cardinal. Suponha que existe $X \in[\mathbb{R}]^{\kappa}$ tal que $X \notin \mathcal{N}$. Então existe $\left(Y_{\xi}\right)_{\xi<\kappa}$ tal que $Y_{\xi} \in \mathcal{M}$ para cada $\xi<\kappa$ e $\mathbb{R}=\bigcup_{\xi<\kappa} Y_{\xi}$.

Estamos prontos para demonstrar a primeira relação entre conjuntos de Luzin e conjuntos de Sierpiński. Assumindo a existência de um conjunto de Luzin podemos construir um conjunto de Sierpiński e vice-versa. Adicionalmente, ambos possuem cardinalidade $\aleph_{1}$.

Teorema 5.1.11 (Rothberger(1938)). Sejam $X, Y \subseteq \mathbb{R}$. Suponha que $X$ é um conjunto de Luzin e $Y$ é um conjunto de Sierpiński. Então temos:

$$
|X|=|Y|=\aleph_{1} .
$$

Demonstração. Seja $X_{0} \subseteq X$ tal que $\left|X_{0}\right|=\aleph_{1}$. Pelos Lemas 5.1.7 e 5.1.5 temos que $X_{0} \notin \mathcal{M}$. Pelo Lema 5.1 .9 temos que existe $\left(Y_{\xi}\right)_{\xi<\omega_{1}}$ tal que $Y_{\xi} \in \mathcal{N}$ para cada $\xi<\omega_{1}$ e $\mathbb{R}=\bigcup_{\xi<\omega_{1}} Y_{\xi}$. Observe que:

$$
Y=Y \cap \mathbb{R}=Y \cap\left(\bigcup_{\xi<\omega_{1}} Y_{\xi}\right)=\bigcup_{\xi<\omega_{1}}\left(Y_{\xi} \cap Y\right) .
$$

Como $Y$ é um conjunto de Sierpiński então $\left|Y_{\xi} \cap Y\right| \leq \aleph_{0}$ para cada $\xi<\omega_{1}$. Logo, temos que:

$$
|Y|=\left|\bigcup_{\xi<\omega_{1}}\left(Y_{\xi} \cap Y\right)\right| \leq \sum_{\xi<\omega_{1}}\left|Y_{\xi} \cap Y\right| \leq \sum_{\xi<\omega_{1}} \aleph_{0} \leq \aleph_{1} .
$$


Como $Y$ é um conjunto de Sierpiński então $|Y|>\aleph_{0}$. Logo, $|Y|=\aleph_{1}$.

Analogamente, considere $Y_{0} \subseteq Y$ tal que $\left|Y_{0}\right|=\aleph_{1}$. Pelos Lemas 5.1.8 e 5.1.6 temos que $Y_{0} \notin \mathcal{N}$. Pelo Lema 5.1 .10 temos que existe $\left(Z_{\xi}\right)_{\xi<\omega_{1}}$ tal que $Z_{\xi} \in \mathcal{N}$ para cada $\xi<\omega_{1}$ e $\mathbb{R}=\bigcup_{\xi<\omega_{1}} Z_{\xi}$. Observe que:

$$
X=X \cap \mathbb{R}=X \cap\left(\bigcup_{\xi<\omega_{1}} Z_{\xi}\right)=\bigcup_{\xi<\omega_{1}}\left(Z_{\xi} \cap X\right) .
$$

Como $X$ é um conjunto de Luzin então $\left|Z_{\xi} \cap X\right| \leq \aleph_{0}$ para cada $\xi<\omega_{1}$. Logo, temos que:

$$
|X|=\left|\bigcup_{\xi<\omega_{1}}\left(Z_{\xi} \cap X\right)\right| \leq \sum_{\xi<\omega_{1}}\left|Z_{\xi} \cap X\right| \leq \sum_{\xi<\omega_{1}} \aleph_{0} \leq \aleph_{1} .
$$

Como $X$ é um conjunto de Luzin então $|X|>\aleph_{0}$. Logo, $|X|=\aleph_{1}$.

A proposição a seguir é uma forma de extender um conjunto magro para um outro conjunto magro, essencialmente. Porém, tal extensão não é qualquer. É uma extensão grande, no sentido de que deixa um resíduo de cardinalidade do contínuo.

Proposição 5.1.12. Se $X \in \mathcal{M}$ então existe $Y \in \mathcal{M}$ tal que $X \subseteq Y$ e $|Y \backslash X|=\mathfrak{c}$.

Demonstração. Observe que existe $A \subseteq \mathbb{R} \backslash X$ um $G_{\delta}$ denso de $\mathbb{R}$. Pelo Teorema 2.1.25 existe $C \subseteq A$ conjunto de Cantor. Como $C \subseteq \mathbb{R}$ é raro então $C \in \mathcal{M}$. Considere $Y=X \cup C$.

A Proposição 5.1 .13 também diz respeito a extensões de conjuntos. É uma maneira de extender um conjunto de medida zero para um outro conjunto de medida zero. Novamente, tal estensão não é arbitrária. É uma extensão grande, no sentido de que deixa um resíduo de cardinalidade do contínuo.

Proposição 5.1.13. Se $M \in \mathcal{N}$ existe $N \in \mathcal{N}$ tal que $M \subseteq N$ e $|N \backslash M|=\mathfrak{c}$.

Demonstração. Note que $\mathbb{R} \backslash M \notin \mathcal{N}$. Logo, $|\mathbb{R} \backslash M|>\aleph_{0}$. Pelo Teorema da Decomposição de Cantor-Bendixson existe $\emptyset \neq P \subseteq \mathbb{R} \backslash M$ conjunto perfeito. Pela Proposição 2.1.23 temos que existe $C \subseteq P$ conjunto de Cantor. Defina $N=M \cup C$. 
O resultado abaixo nos diz que assumindo a hipótese $\operatorname{add}(\mathcal{M})=\mathfrak{c}$ podemos construir de maneira indutiva uma base para o ideal dos subconjuntos magros da reta real. Os elementos de tal base são crescentes, no sentido de que cada elemento é uma extensão de seu antecessor imediato e no caso de um ordinal limite, pode ser escrito com uma reunião dos elementos da base que foram construídos antes dele. Além disso, o resíduo de um elemento da base para seu antecessor imediato possui cardinalidade do contínuo.

Lema 5.1.14. Assuma add $(\mathcal{M})=\mathfrak{c}$. Existe $\left\{X_{\alpha}: \alpha<\mathfrak{c}\right\}$ base de $\mathcal{M}$ tal que $X_{\alpha} \subseteq X_{\beta}$ para cada $\alpha<\beta<\mathfrak{c} e\left|X_{\alpha+1} \backslash X_{\alpha}\right|=\mathfrak{c}$ para cada $\alpha<\mathfrak{c}$. Além disso, para cada $\alpha<\mathfrak{c}$ ordinal limite temos $X_{\alpha}=\bigcup_{\beta<\alpha} X_{\beta}$.

Demonstração. Seja $\left\{Y_{\alpha}: \alpha<\mathfrak{c}\right\}$ base de $\mathcal{M}$ dada pela Proposição 2.2.30. Para construir $\left\{X_{\alpha}: \alpha<\mathfrak{c}\right\}$ iremos proceder por recursão:

- $X_{0}=Y_{0}$

- Fixe $\alpha<\mathfrak{c}$ arbitrário. Suponha construído $X_{\beta} \subseteq \mathbb{R}$ magro para cada $\beta<\alpha$ tal que $X_{\beta} \cup Y_{\beta} \subseteq X_{\beta+1},\left|X_{\beta+1} \backslash X_{\beta}\right|=\mathfrak{c}$ para cada $\beta<\alpha$ e $X_{\beta}=\bigcup_{\gamma<\beta} X_{\gamma}$ se $\beta<\alpha$ for um número ordinal limite. Devemos construir $X_{\alpha} \subseteq \mathbb{R}$. Vamos analisar dois casos:

(i) Suponha que $\alpha=\gamma+1$. Por hipótese de indução temos que $X_{\gamma} \subseteq \mathbb{R}$ é magro. Como $Y_{\gamma} \subseteq \mathbb{R}$ é magro então $X_{\gamma} \cup Y_{\gamma} \subseteq \mathbb{R}$ é magro. Pela Proposição 5.1.12 existe $X_{\gamma+1} \subseteq \mathbb{R}$ magro tal que $X_{\gamma} \cup Y_{\gamma} \subseteq X_{\gamma+1}$ e $\left|X_{\gamma+1} \backslash\left(X_{\gamma} \cup Y_{\gamma}\right)\right|=\mathfrak{c}$. Como $X_{\gamma} \subseteq X_{\gamma} \cup Y_{\gamma}$ e $\left|X_{\gamma+1} \backslash\left(X_{\gamma} \cup Y_{\gamma}\right)\right|=\mathfrak{c}$ então $\left|X_{\gamma+1} \backslash X_{\gamma}\right|=\mathfrak{c}$.

(ii) Suponha que $\alpha<\mathfrak{c}$ é um número ordinal limite. Defina $X_{\alpha}=\bigcup_{\beta<\alpha} X_{\beta}$. Observe que $\left|\left\{X_{\beta}: \beta<\alpha\right\}\right| \leq \alpha<\mathfrak{c}$. Por hipótese de indução temos que $X_{\beta} \subseteq$ $\mathbb{R}$ é magro para cada $\beta<\alpha$. Como add $(\mathcal{M})=\mathfrak{c}$ então $X_{\alpha}=\bigcup_{\beta<\alpha} X_{\beta} \subseteq \mathbb{R}$ é magro.

Por construção temos que $\left\{X_{\alpha}: \alpha<\mathfrak{c}\right\}$ é base de $\mathcal{M}$ e satisfaz as propriedades desejadas.

Com demonstração análoga à do Lema 5.1 .14 também pode-se mostrar o seguinte resultado: 
Lema 5.1.15. Assuma add $(\mathcal{N})=\mathfrak{c}$. Existe $\left\{M_{\alpha}: \alpha<\mathfrak{c}\right\}$ base de $\mathcal{N}$ tal que $M_{\alpha} \subseteq M_{\beta}$ para cada $\alpha<\beta<\mathfrak{c} e\left|M_{\alpha+1} \backslash M_{\alpha}\right|=\mathfrak{c}$ para cada $\alpha<\mathfrak{c}$. Além disso, para cada $\alpha<\mathfrak{c}$ ordinal limite temos $M_{\alpha}=\bigcup_{\beta<\alpha} M_{\beta}$.

Apresentamos abaixo o Teorema de Erdős-Sierpiński, o resultado central desta seção. Seu enunciado original foi feito assumindo-se a Hipótese do Contínuo. Porém, apresentaremos aqui uma modificação, ao invés de assumirmos a Hipótese do Contínuo, assumiremos a hipótese $\operatorname{add}(\mathcal{M})=\operatorname{add}(\mathcal{N})=\mathfrak{c}$ que é mais fraca.

Teorema 5.1.16. Assuma $\operatorname{add}(\mathcal{M})=\operatorname{add}(\mathcal{N})=\mathfrak{c}$. Existe $f: \mathbb{R} \longrightarrow \mathbb{R}$ função bijetora tal que para cada $A \subseteq \mathbb{R}$ temos:

(i) $f[A] \in \mathcal{M} \longleftrightarrow A \in \mathcal{N}$;

(ii) $f[A] \in \mathcal{N} \longleftrightarrow A \in \mathcal{M}$.

Demonstração. Considere $\left\{X_{\alpha}: \alpha<\mathfrak{c}\right\}$ base de $\mathcal{M}$ dada pelo Lema 5.1.15 e $\left\{M_{\alpha}: \alpha<\right.$ $\mathfrak{c}\}$ base de $\mathcal{N}$ dada pelo Lema 5.1.3. Pelo Teorema 5.1.3 podemos supor que $X_{0} \cap M_{0}=\emptyset$ e $X_{0} \cup M_{0}=\mathbb{R}$. Como $\left|X_{\alpha+1} \backslash X_{\alpha}\right|=\left|M_{\alpha+1} \backslash M_{\alpha}\right|=\mathfrak{c}$ para cada $\alpha<\mathfrak{c}$ (Lema 5.1.14 e Lema 5.1.15) então existe $g_{\alpha}: X_{\alpha+1} \backslash X_{\alpha} \longrightarrow M_{\alpha+1} \backslash M_{\alpha}$ função bijetora para cada $\alpha<\mathfrak{c}$. Defina $g=\bigcup_{\alpha<\mathfrak{c}} g_{\alpha}$.

Afirmação 5.1.17. $g$ é uma função $\operatorname{com} \operatorname{dom}(g)=M_{0}$ e $\operatorname{Im}(g)=X_{0}$.

Demonstração. Suponha que $\alpha<\beta<\mathfrak{c}$. Então $\alpha+1 \leq \beta$. Logo, $X_{\alpha+1} \subseteq X_{\beta}$. Portanto, $\left(X_{\beta+1} \backslash X_{\beta}\right) \cap\left(X_{\alpha+1} \backslash X_{\alpha}\right)=\emptyset$. Como $M_{\alpha+1} \subseteq M_{\beta}$ então $\left(M_{\beta+1} \backslash M_{\beta}\right) \cap\left(M_{\alpha+1} \backslash M_{\alpha}\right)=$ $\emptyset$. Como $g_{\alpha}: X_{\alpha+1} \backslash X_{\alpha} \longrightarrow M_{\alpha+1} \backslash M_{\alpha}$ e $g_{\beta}: X_{\beta+1} \backslash X_{\beta} \longrightarrow M_{\beta+1} \backslash M_{\beta}$ então temos que $\operatorname{dom}\left(g_{\alpha}\right) \cap \operatorname{dom}\left(g_{\beta}\right)=\emptyset$ e $\operatorname{Im}\left(g_{\alpha}\right) \cap \operatorname{Im}\left(g_{\beta}\right)=\emptyset$. Logo, $g_{\alpha} \cap g_{\beta}=\emptyset$.

Portanto, $g_{\alpha} \cap g_{\beta}=\emptyset$ para cada $\alpha, \beta<\mathfrak{c}$ tais que $\alpha \neq \beta$. Então, $g=\bigcup_{\alpha<\mathfrak{c}} g_{\alpha}$ é uma função. Observe que $\operatorname{dom}(g)=\bigcup_{\alpha<\mathfrak{c}}\left(X_{\alpha+1} \backslash X_{\alpha}\right)=M_{0}$ e $i m(g)=\bigcup_{\alpha<\mathfrak{c}}\left(M_{\alpha+1} \backslash M_{\alpha}\right)=$ $X_{0}$.

Como $g_{\alpha}: X_{\alpha+1} \backslash X_{\alpha} \longrightarrow M_{\alpha+1} \backslash M_{\alpha}$ é bijetora para cada $\alpha<\mathfrak{c}$ então $g: M_{0} \longrightarrow X_{0}$ é bijetora. Logo, existe $g^{-1}: X_{0} \longrightarrow M_{0}$. Defina $f=g \cup g^{-1}$. Observe que $f: \mathbb{R} \longrightarrow \mathbb{R}$ é uma função bijetora.

Afirmação 5.1.18. $f[A] \in \mathcal{N} \longleftrightarrow A \in \mathcal{M}$. 
Demonstração. Seja $A \subseteq \mathbb{R}$ conjunto magro. Como $\left\{X_{\alpha}: \alpha<\mathfrak{c}\right\}$ é base de $\mathcal{M}$ então existe $\alpha<\mathfrak{c}$ tal que $A \subseteq X_{\alpha}$. Logo, $f[A] \subseteq f\left[X_{\alpha}\right]$. Pela construção de $f$ temos que $f\left[X_{\alpha}\right] \subseteq M_{\alpha} \cap X_{0}$. Como $M_{\alpha} \cap X_{0} \subseteq \mathbb{R}$ possui medida de Lebesgue igual a zero então $f\left[X_{\alpha}\right] \subseteq \mathbb{R}$ possui medida de Lebesgue igual a zero. Portanto, $f[A] \subseteq \mathbb{R}$ possui medida de Lebesgue igual a zero.

Defina $B=f[A]$. Suponha que $B \subseteq \mathbb{R}$ possui medida de Lebesgue igual a zero. Como $\left\{M_{\alpha}: \alpha<\mathfrak{c}\right\}$ é base de $\mathcal{M}$ então existe $\alpha<\mathfrak{c}$ tal que $B \subseteq M_{\alpha}$. Logo, $f^{-1}[B] \subseteq f^{-1}\left[M_{\alpha}\right]$. Pela construção de $f$ temos que $f^{-1}\left[M_{\alpha}\right] \subseteq X_{\alpha} \cap M_{0}$. Como $X_{\alpha} \cap M_{0} \subseteq \mathbb{R}$ é um conjunto magro então $f^{-1}\left[M_{\alpha}\right] \subseteq \mathbb{R}$ é um conjunto magro. Portanto, $A=f^{-1}[B] \subseteq \mathbb{R}$ é um conjunto magro.

A demonstração de $f[A] \in \mathcal{M} \longleftrightarrow A \in \mathcal{N}$ é análoga a 5.1.18

O Corolário 5.1.19 nos dá a segunda relação entre conjuntos de Luzin e conjuntos de Sierpiński, que desta vez será expressa por uma função bijetora de $\mathbb{R}$ em $\mathbb{R}$. Novamente, a hipótese adicional aqui é $\operatorname{add}(\mathcal{N})=\operatorname{add}(\mathcal{M})=\mathfrak{c}$. Porém, incialmente esse resultado foi obtido através da Hipótese do Contínuo, uma vez que a função utilizada aqui é dada pelo Teorema de Erdôs-Sierpiński.

Corolário 5.1.19. Assuma add $(\mathcal{N})=\operatorname{add}(\mathcal{M})=\mathfrak{c}$. Existe $f: \mathbb{R} \longrightarrow \mathbb{R}$ função bijetora tal que:

1. se $L \subseteq \mathbb{R}$ é conjunto de Luzin então $f[L]$ é um conjunto de Sierpiński

2. se $S \subseteq \mathbb{R}$ é conjunto de Sierpiński então $f[S]$ é um conjunto de Luzin.

Demonstração. Demonstraremos o item 1. O item 2 pode ser provado de forma análoga. Considere $f$ dada pelo Teorema 5.1.16. Suponha que $L \subseteq \mathbb{R}$ é conjunto de Luzin. Como $f$ é uma função bijetora e $|L|>\aleph_{0}$ então $|f[L]|>\aleph_{0}$. Considere $A \in \mathcal{N}$ arbitrário. Como $L$ é um conjunto de Luzin então $\left|f^{-1}[A] \cap L\right| \leq \aleph_{0}$. Como $f$ é bijetora então $\left|f^{-1}[A] \cap L\right|=|A \cap f[L]|$. Logo, $|A \cap f[L]| \leq \aleph_{0}$. 


\subsection{Conjectura Dual de Borel}

O objetivo central desta seção é apresentar uma demonstração de que a Conjectura Dual de Borel é independente de ZFC. Como o nome sugere a Conjectura Dual de Borel é uma afirmação dual à Conjectura de Borel no seguinte aspecto: ao invés de trabalharmos com conjuntos fortemente nulos iremos trabalhar com o conceito de conjuntos fortemente magros na formulação da afirmação.

$\mathrm{Na}$ seção anterior apresentamos a dualidade existente entre subconjuntos de medida zero e subconjuntos magros da reta real. O Teorema de Galvin-Mycielski-Solovay nos dá uma caracterização dos subconjuntos fortemente nulos da reta real através de translações. Utilizando esse resultado e a dualidade aparente entre subconjuntos de medida zero e subconjuntos magros da reta real podemos estabeler o conceito de conjunto fortemente magro.

Definição 5.2.1. Seja $X \subseteq \mathbb{R}$. Dizemos que $X$ é fortemente magro se para cada $Y \in \mathcal{N}$ existe $x \in \mathbb{R}$ tal que $(X+x) \cap Y=\emptyset$.

Denotaremos por $\mathcal{S} \mathcal{M}$ o conjunto $\{X \subseteq \mathbb{R}: X$ é fortemente magro $\}$. O Lema 3.3.3 nos permite formular uma noção equivalente da Definição 5.2 .1 .

$$
X \in \mathcal{S M} \longleftrightarrow \forall N \in \mathcal{N}(X+N \neq \mathbb{R})
$$

As Proposições 5.2 .2 e 5.2 .3 nos mostram que a definição de conjunto fortemente magro está, de fato, introduzindo um conceito novo uma vez que extende estritamente o conceito de conjunto magro.

Proposição 5.2.2. Se $X \in \mathcal{S} \mathcal{M}$ então $X \in \mathcal{M}$.

Demonstração. Suponha por absurdo que existe $X \in \mathcal{S M}$ tal que e $X \notin \mathcal{M}$. Sejam $A, B \subseteq \mathbb{R}$ como no Teorema 5.1.3. Demonstraremos que $X+x \nsubseteq B$ para cada $x \in \mathbb{R}$. De fato, suponha por absurdo que existe $x \in \mathbb{R}$ tal que $X+x \subseteq B$. Como $B \in \mathcal{M}$ e $X+x \subseteq B$ então $X+x \in \mathcal{M}$. Então $(X+x)-x \in \mathcal{M}$. Contradição, pois $(X+x)-x=X$.

Como $X+x \nsubseteq B$ para cada $x \in \mathbb{R}$ e $\mathbb{R}=A \cup B$ então $(X+x) \cap A \neq \emptyset$ para cada $x \in \mathbb{R}$. Portanto, não existe $x \in \mathbb{R}$ tal que $(X+x) \cap A=\emptyset$. Logo, $X \notin \mathcal{S M}$. Contradição. 
Proposição 5.2.3. Existe $X \in \mathcal{M}$ tal que e $X \notin \mathcal{S M}$.

Demonstração. Suponha por absurdo que para cada $X \subseteq \mathbb{R}$ magro temos que $X$ é fortemente magro. Sejam $A, B \subseteq \mathbb{R}$ como no Teorema 5.1.3. Como $A \cup B=\mathbb{R}$ então $0 \in A$ ou $0 \in B$. Suponha que $0 \in A$. Defina $B_{0}=B \cup\{0\}$. Note que $A \cup B_{0}=\mathbb{R}$. Como $0 \in A \cap B_{0}$ então $A \cup B_{0} \subseteq A+B_{0}$. Portanto, $A+B_{0}=\mathbb{R}$. Contradição, pois $A$ é nulo.

A Conjectura Dual de Borel é a afirmação (denotada por DBC):

$$
X \in \mathcal{S M} \longleftrightarrow|X| \leq \aleph_{0}
$$

Carlson em 1993 ([5]) enunciou a Conjectura Dual de Borel. No mesmo artigo, demonstra que tal afirmação é consistente com ZFC.

Assumindo a hipótese $\operatorname{cof}(\mathcal{N}) \leq \operatorname{cov}(\mathcal{N})$ iremos construir um subconjunto fortemente magro da reta real com cardinalidade $\operatorname{cof}(\mathcal{N})$. Como $\operatorname{cof}(\mathcal{N})$ é um cardinal não enumerável tal construção nos fornece uma prova de que a negação da Conjectura Dual de Borel é uma afirmação consistente com ZFC.

Abaixo apresentamos um lema auxiliar. Assumindo a hipótese $\operatorname{cof}(\mathcal{N}) \leq \operatorname{cov}(\mathcal{N})$ tal lema nos mostra como construir de maneira indutiva um conjunto (candidato a conjunto fortemente magro) e translações que nível a nível vão escapando de interseções com os elementos de uma base para o ideal dos subconjuntos de medida zero da reta real.

Lema 5.2.4. Assuma que $\operatorname{cof}(\mathcal{N}) \leq \operatorname{cov}(\mathcal{N})$. Se $\left\{B_{\zeta}: \zeta<\operatorname{cof}(\mathcal{N})\right\}$ é uma base de $\mathcal{N}$ então existem $\left\{x_{\xi}: \xi<\operatorname{cof}(\mathcal{N})\right\}$ e $\left\{y_{\xi}: \xi<\operatorname{cof}(\mathcal{N})\right\}$ tais que $\left(\left\{x_{\xi}: \xi<\right.\right.$ $\left.\operatorname{cof}(\mathcal{N})\}+y_{\zeta}\right) \cap B_{\zeta}=\emptyset$ para cada $\zeta<\operatorname{cof}(\mathcal{N})$.

Demonstração. Fixe $\eta<\operatorname{cof}(\mathcal{N})$ arbitrário. Suponha construídos $x_{\xi}, y_{\xi} \in \mathbb{R}$ para cada $\xi<\eta$ tais que $\left(\left\{x_{\xi}: \xi<\eta\right\}+y_{\zeta}\right) \cap B_{\zeta}=\emptyset$ para cada $\zeta<\eta$.

Defina $C_{\eta}=\bigcup_{\xi<\eta, \zeta \leq \eta}\left(B_{\zeta}-x_{\xi}\right)$. Como $B_{\zeta} \in \mathcal{N}$ para cada $\zeta \leq \eta$ então $B_{\zeta}-x_{\xi} \in \mathcal{N}$ para cada $\xi<\eta$. Como $\xi<\eta, \zeta \leq \eta$ então temos:

$$
\mid\left\{B_{\zeta}-x_{\xi}: \xi<\eta \text { e } \zeta \leq \eta\right\}|\leq| \eta \mid<\operatorname{cof}(\mathcal{N}) \leq \operatorname{cov}(\mathcal{N})
$$

Logo, $\bigcup_{\xi<\eta, \zeta \leq \eta}\left(B_{\zeta}-x_{\xi}\right) \neq \mathbb{R}$. Então existe $y_{\eta} \in \mathbb{R} \backslash C_{\eta}$.

Demonstraremos que $\left(\left\{x_{\xi}: \xi<\eta\right\}+y_{\zeta}\right) \cap B_{\zeta}=\emptyset$ para cada $\zeta \leq \eta$. De fato, se $\zeta<\eta$ então por hipótese de indução temos a afirmação. Vamos estudar o caso em que 
$\zeta=\eta$. Suponha por absurdo que $\left(\left\{x_{\xi}: \xi<\eta\right\}+y_{\zeta}\right) \cap B_{\zeta} \neq \emptyset$. Então existe $\xi<\eta$ tal que $x_{\xi}+y_{\zeta} \in B_{\zeta}$. Logo, $y_{\zeta} \in B_{\zeta}-x_{\xi}$. Como $B_{\zeta}-x_{\xi} \subseteq \bigcup_{\xi<\eta, \zeta \leq \eta}\left(B_{\zeta}-x_{\xi}\right)=C_{\eta}$ então $y_{\zeta} \in C_{\eta}$. Contradição.

Note que $\left|\left\{B_{\zeta}-y_{\zeta}: \zeta \leq \eta\right\}\right| \leq|\eta|<\operatorname{cof}(\mathcal{N}) \leq \operatorname{cov}(\mathcal{N})$. Como $B_{\zeta}-y_{\zeta} \in \mathcal{N}$ para cada $\zeta \leq \eta$ e $\eta<\operatorname{cov}(\mathcal{N})$ então $\bigcup_{\zeta \leq \eta}\left(B_{\zeta}-y_{\zeta}\right) \neq \mathbb{R}$. Logo, existe $x_{\eta} \in \mathbb{R} \backslash \bigcup_{\zeta \leq \eta}\left(B_{\zeta}-y_{\zeta}\right)$.

Afirmamos que $\left(\left\{x_{\xi}: \xi \leq \eta\right\}+y_{\zeta}\right) \cap B_{\zeta}=\emptyset$ para cada $\zeta \leq \eta$. Com efeito, se $\zeta<\eta$ então por hipótese de indução temos a afirmação. Suponha que $\zeta=\eta$. Como $x_{\eta} \in \mathbb{R} \backslash \bigcup_{\zeta \leq \eta}\left(B_{\zeta}-y_{\zeta}\right)$ então $x_{\eta} \notin B_{\zeta}-y_{\zeta}$ para cada $\zeta \leq \eta$. Logo, $x_{\eta}+y_{\zeta} \notin B_{\zeta}$ para cada $\zeta \leq \eta$.

O Teorema 5.2.5 nos mostra que a negação da Conjectura Dual de Borel é uma afirmação consistente com ZFC.

Teorema 5.2.5. Assuma $\operatorname{cof}(\mathcal{N}) \leq \operatorname{cov}(\mathcal{N})$. Existe $X \in \mathcal{S} \mathcal{M}$ tal que $|X|=\operatorname{cof}(\mathcal{N})$.

Demonstração. Considere $\mathcal{B}$ uma base de cardinalidade $\operatorname{cof}(\mathcal{N})$ para $\mathcal{N}$. Seja $\left\{B_{\zeta}\right.$ : $\zeta<\operatorname{cof}(\mathcal{N})\}$ uma indexação sem repetições de $\mathcal{B}$. Pelo Lema 5.2 .4 existem $\left\{x_{\xi}: \xi<\right.$ $\operatorname{cof}(\mathcal{N})\}$ e $\left\{y_{\xi}: \xi<\operatorname{cof}(\mathcal{N})\right\}$ tais que $\left(\left\{x_{\xi}: \xi<\operatorname{cof}(\mathcal{N})\right\}+y_{\zeta}\right) \cap B_{\zeta}=\emptyset$ para cada $\zeta<\operatorname{cof}(\mathcal{N})$. Defina $X=\left\{x_{\xi}: \xi<\operatorname{cof}(\mathcal{N})\right\}$. Pela construção realizada em 5.2 .4 temos $|X|=\operatorname{cof}(\mathcal{N})$.

Afirmamos que $X \in \mathcal{S M}$. Com efeito, considere $G \in \mathcal{N}$ arbitrário. Como $\mathcal{B}$ é uma base de $\mathcal{N}$ então existe $\zeta<\operatorname{cof}(\mathcal{N})$ tal que $G \subseteq B_{\zeta}$. Pelo Lema 5.2.4, $y_{\zeta} \in \mathbb{R}$ é tal que $\left(X+y_{\zeta}\right) \cap B_{\zeta}=\emptyset$. Portanto, $\left(X+y_{\zeta}\right) \cap G=\emptyset$.

No que segue iremos trabalhar para apresentar uma demonstração de que a Conjectura Dual de Borel é uma afirmação consistente com ZFC. Na verdade, demonstraremos que uma afirmação equivalente à Conjectura Dual de Borel é consistente com ZFC. Tal afirmação é a Conjectura Dual de Borel em $\mathbb{K}$.

Definição 5.2.6. Dizemos que $X \subseteq \mathbb{K}$ é fortemente magro em $\mathbb{K}$ se para cada $N \in$ $\mathcal{N}_{\mathbb{K}}$ existe $x \in \mathbb{K}$ tal que $(X+x) \cap N=\emptyset$. Denotaremos por $\mathcal{S M}_{\mathbb{K}}$ o conjunto $\{X \subseteq \mathbb{K}: X$ é fortemente magro $\}$. Usaremos $D B C_{\mathbb{K}}$ para denotar Conjetura Dual de Borel em $\mathbb{K}$ que é a afirmação:

Se $X \in \mathcal{S M}_{\mathbb{K}}$ então $|X| \leq \aleph_{0}$. 
O resultado abaixo nos permite trabalhar com a Conjectura Dual de Borel $\mathbb{K}$ no que segue.

Teorema 5.2.7. $D B C_{\mathbb{K}} \longleftrightarrow D B C$.

Demonstração. $(\longrightarrow)$ Considere $X \in \mathcal{S} \mathcal{M}$. Defina $X_{n}=X \cap[n, n+1[$ para cada $n \in \mathbb{Z}$. Note que $X_{n} \in \mathcal{S} \mathcal{M}$ para cada $n \in \mathbb{Z}$. Definimos $f: \mathbb{R} \longrightarrow \mathbb{K}$ como sendo $f(x)=x-\lfloor x\rfloor$ para cada $x \in \mathbb{R}$. Observe que $f\left[X_{n}\right] \in \mathcal{S} \mathcal{M}_{\mathbb{K}}$ para cada $n \in \mathbb{Z}$. De fato, $f\left[X_{n}\right]=X_{n}-n$ para cada $n \in \mathbb{Z}$. Como $X_{n} \in \mathcal{S M}$ para cada $n \in \mathbb{Z}$ então $X_{n}-n \in \mathcal{S M}_{\mathbb{K}}$ para cada $n \in \mathbb{Z}$.

Por hipótese, temos que $\left|X_{n}\right| \leq \aleph_{0}$ para cada $n \in \mathbb{Z}$. Como $X=\bigcup_{n \in \mathbb{Z}} f^{-1}\left[X_{n}\right]$ então $|X| \leq \aleph_{0}$.

$(\longleftarrow)$ Considere $X \in \mathcal{S M}_{\mathbb{K}}$. Fixe $N \in \mathcal{N}$ arbitrário. Definimos $N_{k}=N \cap[k, k+1[$ para cada $k \in \mathbb{Z}$. Observe que $N_{k} \in \mathcal{N}_{k}$ para cada $k \in \mathbb{Z}$.

Note que $f\left[N_{k}\right] \in \mathcal{N}_{\mathbb{K}}$ para cada $k \in \mathbb{Z}$. De fato, $f\left[N_{k}\right]=N_{k}-k$ para cada $k \in \mathbb{Z}$. Como a medida de Lebesgue é invariante por translações então $N_{k}-k \in \mathcal{N}_{\mathbb{K}}$

Considere $M=\bigcup_{n \in \mathbb{Z}} f\left[N_{k}\right]$. Observe que $M \in \mathcal{N}_{\mathbb{K}}$. Como $X \in \mathcal{S M}_{\mathbb{K}}$ então existe $x \in \mathbb{K}$ tal que $(X+x) \cap M=\emptyset$.

Afirmamos que $(X+x) \cap N=\emptyset$. De fato, suponha por absurdo que $(X+x) \cap N \neq \emptyset$. Seja $z \in(X+x) \cap N$. Considere $y \in X$ tal que $z=y+x \in N$. Como $N=\bigcup_{n \in \mathbb{Z}} N_{k}$ e $z \in N$ então existe $k_{0} \in \mathbb{Z}$ tal que $z \in N_{k_{0}}$. Por um lado, temos que $f(z) \in f\left[N_{k_{0}}\right] \subseteq M$. Por outro, lado $f(z)=y+x \in X+x$. Contradição, pois $(X+x) \cap M=\emptyset$.

Logo, $X \in \mathcal{S M}$. Então, $|X| \leq \aleph_{0}$.

Apresentamos abaixo um resultado referente a Teoria Aditiva dos Números. A prova de tal resultado foge dos nossos objetivos. Tal resultado, juntamente com o Corolário 5.2 .9 nos auxiliará a construir uma determinada ordem parcial para utilizarmos a técnica de forcing.

Teorema 5.2.8 (Lorentz, [11]). Existe uma constante $C$ tal que para cada $n \in \omega$ e para cada $\left\{\overline{a_{0}}, \overline{a_{1}}, \ldots, \overline{a_{j}}\right\} \subseteq \mathbb{Z}_{n}$ temos que existe $\left\{\overline{b_{0}}, \overline{b_{1}}, \ldots, \overline{b_{k}}\right\} \subseteq \mathbb{Z}_{n}$ com:

$$
k \leq \frac{C \cdot n \cdot \log (j)}{j}
$$

Além disso, temos que para cada $\bar{x} \in \mathbb{Z}_{n}$ existem $i \in\{0,1, \ldots, j\}$ e $l \in\{0,1, \ldots, k\}$ tais que $\bar{x}=\overline{a_{i}}+\overline{b_{l}}$. 
Corolário 5.2.9. Existe uma constante $C$ tal que para cada $n \in \omega$ com $n>1$ e para cada $A \subseteq \mathbb{Z}_{n}$ com $|A| \geq 2$ existe $B \subseteq \mathbb{Z}_{n}$ tal que $A+B=\mathbb{Z}_{n}$ e $|B| \leq \frac{C \cdot n \cdot \log (|A|)}{|A|}$.

Demonstração. Decorre imediatamente do Teorema 5.2.8.

Apresentamos abaixo uma maneira de escrever $\mathbb{K}$ como soma de um conjunto finito com cardinalidade maior do que dois e uma reunião de intervalos abertos com extremos racionais que possui sua medida majorada em função do conjunto finito inicial. Tal resultado é necessário para construirmos uma ordem parcial.

Proposição 5.2.10. Suponha que $X \subseteq \mathbb{K}$ é finito e $|X| \geq 2$. Então existe $U \subseteq \mathbb{K}$ aberto em $\mathbb{K}$ tal que $X+U=\mathbb{K}$ e $\mu(U) \leq \frac{2 \cdot C \cdot \log (|X|)}{|X|}$. Além disso, U é reunião finita de intervalos abertos com extremos racionais.

Demonstração. Suponha que $X \subseteq \mathbb{K}$ é finito e $|X| \geq 2$. Como $X$ é finito então existe $m \in \omega \backslash\{0\}$ tal que $\frac{1}{m}<|x-y|$ para cada $x, y \in X$.

Considere o seguinte conjunto $A=\left\{i \in\{0, \ldots, m-1\}: X \cap\left[\frac{i}{m}, \frac{i+1}{m}[\neq \emptyset\}\right.\right.$. Iremos demonstrar que vale a igualdade $|X|=|A|$. De fato, considere $\left\{i_{0}, i_{1}, \ldots, i_{k}\right\}$ enumeração sem repetições de $A$. Afirmamos que existe um único $x_{j} \in X \cap\left[\frac{i_{j}}{m}, \frac{i_{j}+1}{m}[\right.$ para cada $j \in\{0, \ldots, k\}$. Com efeito, a existência decorre diretamente da definição do conjunto $A$. Para demonstrar a unicidade iremos proceder por contradição. Suponha por absurdo que existem $x, y \in X$ com $x \neq y$ tais que $x, y \in\left[\frac{i_{j}}{m}, \frac{i_{j}+1}{m}[\right.$ para algum $j \in\{0, \ldots, k\}$. Então temos $|x-y| \leq \operatorname{diam}\left(\left[\frac{i_{j}}{m}, \frac{i_{j}+1}{m}[)\right.\right.$. Como diam $\left(\left[\frac{i_{j}}{m}, \frac{i_{j}+1}{m}[)=\frac{1}{m}\right.\right.$ então $\frac{1}{m}<|x-y| \leq \frac{1}{m}$. Contradição. Considere $f: A \longrightarrow X$ dada por $f\left(i_{j}\right)=x_{j}$ para cada $j \in\{0, \ldots, k\}$. Como $\left[\frac{i_{j}}{m}, \frac{i_{j}+1}{m}\left[\cap\left[\frac{i_{l}}{m}, \frac{i_{l}+1}{m}[=\emptyset\right.\right.\right.$ para cada $j, l \in\{0, \ldots, k\}$ com $j \neq l$ temos que $f$ é injetora. A sobrejetividade decorre da definição de $f$.

Como $X$ é finito e $|X|=|A|$ então $A$ é finito. Além disso, $|A|=|X| \geq 2$. Defina $\tilde{A}=\left\{\bar{i} \in \mathbb{Z}_{m}: i \in A\right\}$. Como $A$ é finito e $|A| \geq 2$ então $\tilde{A}$ é finito e $|\tilde{A}| \geq 2$. Pelo Corolário 5.2 .9 existe $\tilde{B} \subseteq \mathbb{Z}_{m}$ tal que $\tilde{A}+\tilde{B}=\mathbb{Z}_{m}$ e, além disso, $|\tilde{B}| \leq \frac{C \cdot m \cdot \log (|\tilde{A}|)}{|\tilde{A}|}$ sendo que $C$ é uma constante. Defina:

$$
U=\bigcup\{] \frac{j-1}{m}, \frac{j+1}{m}[: \bar{j} \in \tilde{B}\}
$$


Como a reunião de intervalos abertos de $\mathbb{K}$ é um aberto de $\mathbb{K}$ então temos que $U \subseteq \mathbb{K}$ é um aberto de $\mathbb{K}$. Observe que $U \subseteq \mathbb{K}$ é reunião finita de intervalos com extremos racionais.

Afirmação 5.2.11. Para cada $i \in\{0, \ldots, m-1\}$ existe $j \in\{0, \ldots, m\}$ tal que $\bar{j} \in \tilde{B}$ e existe $x \in X$ satisfazendo $\left[\frac{i}{m}, \frac{i+1}{m}[\subseteq] \frac{j-1}{m}, \frac{j+1}{m}[+x\right.$.

Demonstração. Fixe $i \in\{0, \ldots, m-1\}$ arbitrário. Como $\tilde{A}+\tilde{B}=\mathbb{Z}_{m}$ então existem $l \in\{0,1, \ldots, k\}$ e $\bar{j} \in \tilde{B}$ tais que $\bar{i}=\overline{i_{l}}+\bar{j}$. Pela escolha de $x_{l}$ temos que:

$$
\left[\frac{i}{m}, \frac{i+1}{m}[\subseteq] \frac{j-1}{m}, \frac{j+1}{m}\left[+x_{l} .\right.\right.
$$

Iremos demonstrar que $X+U=\mathbb{K}$. De fato, considere $y \in \mathbb{K}$ arbitrário. Como $\mathbb{K}=\bigcup_{i=0}^{m-1}\left[\frac{i}{m}, \frac{i+1}{m}\left[\right.\right.$ então existe $i \in\{0, \ldots, m\}$ tal que $y \in\left[\frac{i}{m}, \frac{i+1}{m}[\right.$. Pela Afirmação 5.2 .11 temos que existe $j \in\{0, \ldots, m\}$ tal que $\bar{j} \in \tilde{B}$ e existe $x \in X$ satisfazendo $\left[\frac{i}{m}, \frac{i+1}{m}[\subseteq] \frac{j-1}{m}, \frac{j+1}{m}[+x\right.$. Logo, $y \in] \frac{j-1}{m}, \frac{j+1}{m}[+x$. Portanto, existe $z \in] \frac{j-1}{m}, \frac{j+1}{m}[$ tal que $y=z+x$.

Demonstraremos que $\mu(U) \leq \frac{2 \cdot C \cdot \log (|X|)}{|X|}$. Com efeito, observe que $|\tilde{A}|=|A|$. Como $|X|=|A|$ então $|\tilde{A}|=|X|$. Logo, temos:

$$
\begin{aligned}
\mu(U) & =\mu\left(\bigcup\{] \frac{j-1}{m}, \frac{j+1}{m}[: \bar{j} \in \tilde{B}\}\right) \\
& \leq \sum_{\bar{j} \in \tilde{B}} \mu(\rfloor \frac{j-1}{m}, \frac{j+1}{m}\lfloor) \\
& =\sum_{\bar{j} \in \tilde{B}}\left(\frac{2}{m}\right) \\
& =|\tilde{B}| \cdot \frac{2}{m} \\
& \leq\left(C \cdot m \cdot \frac{\log (|\tilde{A}|)}{|\tilde{A}|}\right) \cdot \frac{2}{m} \\
& =2 \cdot C \cdot \frac{\log (|X|)}{|X|} .
\end{aligned}
$$


Abaixo apresentamos uma demonstração de que em todo grupo abeliano que pode ser escrito como soma de dois subconjuntos, as translações de um somando feitas por um elemento do grupo não encontram o oposto do outro somando. Tal fato é utilizado na Proposição 5.2.13. mais adiante.

Lema 5.2.12. Seja $(G,+)$ um grupo abeliano. Se $A, B \subseteq G$, são tais que $A+B=G$ então $(A+g) \cap(-B) \neq \emptyset$ para cada $g \in G$.

Demonstração. Fixe $g \in G$ arbitrário. Como $G$ é um grupo então existe $-g \in G$. Por hipótese, temos que $A+B=G$. Então existem $a \in A$ e $b \in B$ tais que $-g=a+b$. Note que $a+g=-b$. Como $-b \in(-B)$ então $a+g \in(-B)$. Logo, $a+g \in(A+g) \cap(-B)$.

A Proposição 5.2.13 nos dá uma maneira de construir uma reunião finita de intervalos fechados com extremos racionais que escapa de todas as translações feitas por elementos de $\mathbb{K}$ de um conjunto finito com cardinalidade maior do que dois dado e, além disso, sua medida majora um derterminado número real que pde ser escrito em função do conjunto finito.

Proposição 5.2.13. Suponha que $X \subseteq \mathbb{K}$ é finito e $|X| \geq 2$. Então existe $P \subseteq \mathbb{K}$ tal que $X \not \subset P+x$ para cada $x \in \mathbb{K}$ e $\mu(P) \geq 1-\frac{2 \cdot C \cdot \log (|X|)}{|X|}$. Além disso, P é reunião finita de intervalos fechados com extremos racionais.

Demonstração. Como $X \subseteq \mathbb{K}$ é finito e $|X| \geq 2$ então pela Proposição 5.2 .10 existe $U \subseteq \mathbb{K}$ aberto em $\mathbb{K}$ tal que $X+U=\mathbb{K}$ e $\mu(U) \leq \frac{2 \cdot C \cdot \log (|X|)}{|X|}$. Além disso, $U \subseteq \mathbb{K}$ é uma reunião finita de intervalos abertos com extremos racionais. Defina $P=\mathbb{K} \backslash(-U)$.

Como $U \subseteq \mathbb{K}$ é uma reunião finita de intervalos abertos com extremos racionais então $-U$ é uma reunião finita de intervalos abertos com extremos racionais.

Suponha por absurdo que existe $x \in \mathbb{K}$ tal que $X \subseteq P+x$. Observe que:

$$
\begin{aligned}
X \subseteq P+x & \Longleftrightarrow X-x \subseteq P \\
& \Longleftrightarrow(X-x) \cap(\mathbb{K} \backslash P)=\emptyset \\
& \Longleftrightarrow(X-x) \cap(-U)=\emptyset .
\end{aligned}
$$


Contradizendo o Lema 5.2 .12 , Resta demonstrar que $\mu(P) \leq 1-\frac{2 \cdot C \cdot \log (|X|)}{|X|}$. De fato, temos que:

$$
\begin{aligned}
\mu(P) & =\mu(\mathbb{K} \backslash(-U)) \\
& =1-\mu(-U) \\
& =1-\mu(U) \\
& \geq 1-\frac{2 \cdot C \cdot \log (|X|)}{|X|} .
\end{aligned}
$$

Note que $\bullet$ vale pois $\mu(U) \leq \frac{2 \cdot C \cdot \log (|X|)}{|X|}$

Apresentamos abaixo a ordem parical que será utilizada na técnica de forcing para demonstrarmos que existe um modelo que satisfaz a Conjectura de Dual Borel em $\mathbb{K}$.

Definição 5.2.14. Fixe $c \in \mathbb{K}$ arbitrário. Defina:

$\mathbb{P}_{c}=\left\{p \subseteq \mathbb{K}: p=\bigcup_{i=0}^{n}\left[a_{i}, b_{i}\right]\right.$ com $a_{i}, b_{i} \in \mathbb{Q}$ para cada $i \in\{0, \ldots, n\}, n \in \omega$ e $\left.\mu(p)>c\right\}$

Consideraremos $\mathbb{P}_{c}$ parcialmente ordenado pela inclusão.

É interessantante em alguns momentos que sucedem que possamos supor que os intervalos que compõem um elemento dessa ordem parcial (Definição 5.2.14) sejam dois a dois disjuntos. A observação abaixo vai nesse sentindo.

Observação 5.2.15. Fixe $c \in \mathbb{K}$ arbitrário. Seja $p \in \mathbb{P}_{c}$. Então existem $\left[a_{0}, b_{0}\right], \ldots,\left[a_{n}, b_{n}\right]$ intervalos com extremos racionais tais que $p=\bigcup_{i=0}^{n}\left[a_{i}, b_{i}\right]$. Podemos supor, a menos de menção contrário, que $\left[a_{i}, b_{i}\right] \cap\left[a_{j}, b_{j}\right]=\emptyset$ se $i, j \in\{0,1, \ldots, n\}$ e $i \neq j$.

A ordem parcial apresentada acima é enumerável. Demonstraremos esse fato abaixo.

Proposição 5.2.16. $\mathbb{P}_{c}$ é enumerável para cada $c \in[0,1[$

Demonstração. Para demonstrar o resultado desejado iremos provar que $\aleph_{0} \leq\left|\mathbb{P}_{c}\right|$ e $\left|\mathbb{P}_{c}\right| \leq \aleph_{0}$ 
$\aleph_{0} \leq\left|\mathbb{P}_{c}\right|:$ Como $\mathbb{Q} \cap \mathbb{K}$ é denso em $\mathbb{K}$ então $\left.\mid \mathbb{Q} \cap\right] c, 1\left[\mid=\aleph_{0}\right.$. Seja $\left\{x_{i}: i \in \omega\right\}$ uma enumeração de $\mathbb{Q} \cap] c, 1$ sem repetições. Observe que $\left[0, x_{i}\right] \in \mathbb{P}_{c}$ para cada $i \in \omega$. Como $\left[0, x_{i}\right] \neq\left[0, x_{j}\right]$ para cada $i, j \in \omega$ tais que $i \neq j$ temos que $\left|\mathbb{P}_{c}\right| \geq \aleph_{0}$.

$\left|\mathbb{P}_{c}\right| \leq \aleph_{0}$ : Iremos mostrar que existe uma função injetora $f: \mathbb{P}_{c} \longrightarrow[\mathcal{I}]^{<\omega}$, sendo que $\mathcal{I}$ é o conjunto $\{[a, b]: a, b \in \mathbb{Q}\}$.

Fixe $p \in \mathbb{P}_{c}$. Sejam $\left[a_{0}, b_{0}\right], \ldots,\left[a_{n}, b_{n}\right]$ intervalos fechados extremos racionais tais que $p=\bigcup_{i=0}^{n}\left[a_{i}, b_{i}\right]$. Pela Observação 5.2 .15 podemos supor que $\left[a_{i}, b_{i}\right] \cap\left[a_{j}, b_{j}\right]=\emptyset$ para cada $i, j \in\{0, \ldots, n\}$ tais que $i \neq j$. Defina $f(p)=\left\{\left[a_{0}, b_{0}\right], \ldots,\left[a_{n}, b_{n}\right]\right\}$.

Sejam $p, q \in \mathbb{P}_{c}$ tais que $p \neq q$. Considere $\left[a_{0}, b_{0}\right], \ldots,\left[a_{n}, b_{n}\right],\left[c_{0}, d_{0}\right], \ldots,\left[c_{m}, d_{m}\right]$ intervalos fechados extremos racionais tais que $p=\bigcup_{i=0}^{n}\left[a_{i}, b_{i}\right]$ e $q=\bigcup_{j=0}^{m}\left[c_{j}, d_{j}\right]$. Pela Observação 5.2 .15 podemos supor que $\left[a_{i}, b_{i}\right] \cap\left[a_{l}, b_{l}\right]=\emptyset$ e $\left[c_{j}, d_{j}\right] \cap\left[c_{k}, d_{k}\right]=\emptyset$ para cada $i, j \in\{0, \ldots, n\}$ e $j, k \in\{0, \ldots, m\}$ tais que $i \neq l$ e $j \neq k$. Como $p \neq q$ então existe $x \in \mathbb{K}$ tal que $x \in p$ e $x \notin q$ ou $x \in q$ e $x \notin p$. Iremos supor que $x \in p$ e $x \notin q$. Como $x \in p$ e $p=\bigcup_{i=0}^{n}\left[a_{i}, b_{i}\right]$ então existe $i \in\{0, \ldots, n\}$ tal que $x \in\left[a_{i}, b_{i}\right]$. Como $x \notin q$ e $q=\bigcup_{j=0}^{m}\left[c_{j}, d_{j}\right]$ então $x \notin\left[c_{j}, d_{j}\right]$ para cada $j \in\{0, \ldots, m\}$. Então temos que $\left[a_{i}, b_{i}\right] \neq$ $\left[c_{j}, d_{j}\right]$ para cada $j \in\{0, \ldots, m\}$. Portanto, $\left[a_{i}, b_{i}\right] \notin\left\{\left[c_{0}, d_{0}\right], \ldots,\left[c_{m}, d_{m}\right]\right\}$. Logo, temos que $\left\{\left[a_{0}, b_{0}\right], \ldots,\left[a_{n}, b_{n}\right]\right\} \neq\left\{\left[c_{0}, d_{0}\right], \ldots,\left[c_{m}, d_{m}\right]\right\}$. Como $f(p)=\left\{\left[a_{0}, b_{0}\right], \ldots,\left[a_{n}, b_{n}\right]\right\}$ e $f(q)=\left\{\left[c_{0}, d_{0}\right], \ldots,\left[c_{m}, d_{m}\right]\right\}$ então $f(p) \neq f(q)$.

Logo, $\left|\mathbb{P}_{c}\right| \leq\left|[\mathcal{I}]^{<\omega}\right|$. Sabemos que $|\mathcal{I}|=\aleph_{0}$. Portanto, $\left|[\mathcal{I}]^{<\omega}\right|=\aleph_{0}$. Então $\left|\mathbb{P}_{c}\right| \leq \aleph_{0}$

Abaixo apresentamos uma demonstração de que a ordem parcial que definimos (Definição 5.2.14 é separativa. Portanto, teremos uma ordem parcial enumerável e seperativa. De um modo geral forçar com ordens parciais com essas características é adicionar um real de Cohen na extensão.

Proposição 5.2.17. Fixe $c \in\left[0,1\left[\right.\right.$ arbitrário. Temos que $\mathbb{P}_{c}$ é uma ordem parcial separativa.

Demonstração. Fixe $p \in \mathbb{P}_{c}$ arbitrário. Sejam $\left[a_{0}, b_{0}\right], \ldots,\left[a_{n}, b_{n}\right]$ intervalos fechados de extremos racionais tais que $p=\bigcup_{i=0}^{n}\left[a_{i}, b_{i}\right]$. Pela Observação 5.2 .15 podemos supor que $\left[a_{i}, b_{i}\right] \cap\left[a_{j}, b_{j}\right]=\emptyset$ para cada $i, j \in\{0, \ldots, n\}$ tais que $i \neq j$.

Seja $\delta \in] \frac{(p)-c}{2}, \xi\left[\cap \mathbb{Q}\right.$, sendo que $\xi=\min \left\{\frac{\mu(p)}{2}, \mu(p)-c\right\}$. Defina $\frac{1}{2}<\alpha=\frac{\mu(p)-\delta}{\mu(p)}<$ 1. 
Considere $p^{\prime}=\bigcup_{i=0}^{n}\left[a_{i}, \alpha \cdot\left(b_{i}-a_{i}\right)+a_{i}\right]$ e $p^{\prime \prime}=\bigcup_{i=0}^{n}\left[\alpha \cdot\left(a_{i}-b_{i}\right)+b_{i}, b_{i}\right]$. Como $\frac{1}{2}<\alpha$ então $a_{i}<\alpha \cdot\left(b_{i}-a_{i}\right)+a_{i}$ e $\alpha \cdot\left(a_{i}-b_{i}\right)+b_{i}<b_{i}$ para cada $i \in\{0, \ldots, n\}$.

Note que

$$
\begin{aligned}
\mu\left(p^{\prime}\right) & =\mu\left(\bigcup_{i=0}^{n}\left[a_{i}, \alpha \cdot\left(b_{i}-a_{i}\right)+a_{i}\right]\right) \\
& =\sum_{i=0}^{n}\left(\left(a_{i}+\alpha\left(b_{i}-a_{i}\right)\right)-a_{i}\right) \\
& =\alpha \mu(p) .
\end{aligned}
$$

Como $\alpha \mu(p)=\mu(p)-\delta$ então decorre da escolha de $\delta$ que $\mu(p)-\delta>c$. Podese demonstrar de forma análoga à acima que $\mu\left(p^{\prime \prime}\right)=\mu\left(p^{\prime}\right)$. Resta provarmos que $\mu\left(p^{\prime} \cap p^{\prime \prime}\right) \leq c$. De fato, observe que

$$
\begin{aligned}
\mu\left(\bigcup_{i=0}^{n}\left[\alpha\left(a_{i}-b_{i}\right)+b_{i}, \alpha\left(b_{i}-a_{i}\right)+a_{i}\right]\right) & =\sum_{i=0}^{n}\left(\left(\alpha\left(b_{i}-a_{i}\right)+a_{i}\right)-\left(\alpha\left(a_{i}-b_{i}\right)+b_{i}\right)\right) \\
& =-\mu(p)+\alpha \cdot \sum_{i=0}^{n}\left(\left(b_{i}-a_{i}\right)-\left(a_{i}-b_{i}\right)\right) \\
& =-\mu(p)+2 \alpha \mu(p) .
\end{aligned}
$$

Como $-\mu(p)+2 \alpha \mu(p)=\mu(p)-2 \delta$ então decorre da escolha de $\delta$ que $\mu(p)-2 \delta \leq c$.

O lema abaixo nos mostra que é equivalente forçar com $\mathbb{P}_{c}$ ou $\left(F u n c_{\omega}(\omega, 2), \supset\right)$. Uma vez que essas ordens parciais produzem as mesmas extensões genéricas.

Lema 5.2.18. Fixe $c \in\left[0,1\left[\right.\right.$ arbitrário. As ordens parciais $\mathbb{P}_{c}$ e $\left(\right.$ Func $\left._{\omega}(\omega, 2), \supset\right)$ produzem as mesmas extensões genéricas.

Demonstração. Pela Proposição 5.2.16 e pela Proposição 5.2.17 temos que $\mathbb{P}_{c}$ é uma ordem parcial separativa enumerável. Pela Proposição 2.3.9 temos que existe uma imersão densa $\varphi: \widetilde{\mathbb{P}} \longrightarrow \mathbb{P}_{c}$. Pelo Teorema 2.3.2 temos que $\widetilde{\mathbb{P}}$ e $\mathbb{P}_{c}$ produzem as mesmas extensões genéricas. 
Pelo Exemplo 2.3.5 e pela Proposição 2.3.4 temos que $F u n c_{\omega}(\omega, 2)$ é uma ordem parcial separativa enumerável. Pela Proposição 2.3.9 temos que existe uma imersão densa $\psi: \widetilde{\mathbb{P}} \longrightarrow$ Func $_{\omega}(\omega, 2)$. Pelo Teorema 2.3.2 temos que $\widetilde{\mathbb{P}}$ e $F u n c_{\omega}(\omega, 2)$ geram as mesmas extensões genéricas. $\operatorname{Logo}, \mathbb{P}_{c}$ e $F u n c_{\omega}(\omega, 2)$ também fornecem as mesmas extensões genéricas.

Definição 5.2.19. Fixe $c \in \mathbb{K}$ arbitrário. Suponha que $V$ é um modelo transitivo enumerável de $Z F C$ e que $G$ é um filtro $V$-genérico sobre $\mathbb{P}_{c}$. Seja $p \in \mathbb{P}_{c}$. Então $p \subseteq \mathbb{K}$ e existem números racionais $a_{0}, \ldots, a_{n}, b_{0}, \ldots, b_{n}$ tais que $p=\bigcup_{i=0}^{n}\left[a_{i}, b_{i}\right]$. Além disso, $\mu(p)>c$. Definimos o conjunto compacto determinado por $G$ como sendo a interseção $\bigcap G$ de todos $p^{V[G]}$ onde $p \in G$ e $p^{V[G]}$ é o conjunto $\bigcup_{i=0}^{n}\left[a_{i}, b_{i}\right]$ calculado na estensão $V[G]$.

Apresentamos abaixo o cálculo da medida (na extensão) para o compacto determinado por um filtro genérico.

Proposição 5.2.20. Fixe $c \in \mathbb{K}$. Sejam $V$ um modelo transitivo enumerável de $Z F C$ tal que $\mathbb{P}_{c} \in V$ e $G$ um filtro $V$-genérico em $\mathbb{P}_{c}$. O conjunto compacto determinado por $G$ possui medida de Lebesgue igual a c em $V[G]$.

Demonstração. Denote por $K$ o conjunto compacto determinado por $G$ (Definição 5.2 .19 .

- $\mu(K) \leq c$ : Defina $D_{n}=\left\{p \in \mathbb{P}_{c}: \mu(p) \leq c+\frac{1}{n+1}\right\}$ para cada $n \in \omega$. Temos a seguinte:

Afirmação 5.2.21. $D_{n}$ é denso em $\mathbb{P}_{c}$ para cada $n \in \omega$.

Demonstração. Sejam $p \in \mathbb{P}_{c}$ e $n \in \omega$. Considere $\left[a_{0}, b_{0}\right], \ldots,\left[a_{n}, b_{n}\right]$ intervalos fechados com extremos racionais tais que $p=\bigcup_{i=0}^{n}\left[a_{i}, b_{i}\right]$. Pela Observação 5.2.15 podemos supor que $\left[a_{i}, b_{i}\right] \cap\left[a_{j}, b_{j}\right]=\emptyset$ para cada $i, j \in\{0, \ldots, n\}$ tais que $i \neq j$. Iremos analisar dois casos:

$$
\begin{aligned}
\mu(p) \leq c+\frac{1}{n+1}: & \text { Como } p \in \mathbb{P}_{c} \text { então } c<\mu(p) . \text { Logo, } p \in D_{n} . \\
c+\frac{1}{n+1}<\mu(p): & \text { Como } \left.c+\frac{1}{n+1}<\mu(p) \text { então }(\mu(p)-c)-\frac{1}{n+1}>0 \text {. Portanto, }\right](\mu(p)-c)-\frac{1}{n+1}, \mu(p)-c[ \\
& \text { é um intervalo aberto não-vazio em }[0,1[\text {. Como } \mathbb{Q} \cap[0,1[\text { é denso em }[0,1[ \\
& \text { então existe um número racional } x \in](\mu(p)-c)-\frac{1}{n+1}, \mu(p)-c[\text {. Considere }
\end{aligned}
$$


$\varepsilon=\frac{x}{n+1}$. Como $](\mu(p)-c)-\frac{1}{n+1}, \mu(p)-c\left[\subseteq\left[(\mu(p)-c)-\frac{1}{n+1}, \mu(p)-c[\right.\right.$ então $(\mu(p)-c)-\frac{1}{n+1} \leq(n+1) \cdot \varepsilon<\mu(p)-c$. Defina $q=\bigcup_{i=0}^{n}\left[a_{i}+\frac{\varepsilon}{2}, b_{i}-\frac{\varepsilon}{2}\right]$. Note que $\left[a_{i}+\frac{\varepsilon}{2}, b_{i}-\frac{\varepsilon}{2}\right]$ é um intervalo fechado com extremos racionais para cada $i \in\{0, \ldots, n\}$. Observe que:

$$
\begin{aligned}
\mu(q) & =\mu\left(\bigcup_{i=0}^{n}\left[a_{i}+\frac{\varepsilon}{2}, b_{i}-\frac{\varepsilon}{2}\right]\right) \\
& =\sum_{i=0}^{n} \mu\left(\left[a_{i}+\frac{\varepsilon}{2}, b_{i}-\frac{\varepsilon}{2}\right]\right) \\
& =\sum_{i=0}^{n}\left[\left(b_{i}-\frac{\varepsilon}{2}\right)-\left(a_{i}+\frac{\varepsilon}{2}\right)\right] \\
& =\sum_{i=0}^{n}\left(b_{i}-a_{i}\right)-\sum_{i=0}^{n} \varepsilon \\
& =\mu(p)-(n+1) \cdot \varepsilon .
\end{aligned}
$$

Como $(n+1) \cdot \varepsilon<\mu(p)-c$ então $c<\mu(p)-(n+1) \cdot \varepsilon$. Logo, $c<\mu(q)$. Note que $\left[a_{i}+\frac{\varepsilon}{2}, b_{i}-\frac{\varepsilon}{2}\right] \subseteq\left[a_{i}, b_{i}\right]$ para cada $i \in\{0, \ldots, n\}$. Logo, $\bigcup_{i=0}^{n}\left[a_{i}+\frac{\varepsilon}{2}, b_{i}-\frac{\varepsilon}{2}\right] \subseteq$ $\bigcup_{i=0}^{n}\left[a_{i}, b_{i}\right]$. Como $q=\bigcup_{i=0}^{n}\left[a_{i}+\frac{\varepsilon}{2}, b_{i}-\frac{\varepsilon}{2}\right]$ e $p=\bigcup_{i=0}^{n}\left[a_{i}, b_{i}\right]$ então $q \subseteq p$, isto é, $q$ estende $p$. Como $(\mu(p)-c)-\frac{1}{n+1} \leq(n+1) \cdot \varepsilon$ então $\mu(p)-(n+1) \cdot \varepsilon \leq c+\frac{1}{n+1}$. Portanto, $q \in D_{n}$.

Note que $D_{n} \in V$ para cada $n \in \omega$. Como $G$ é um filtro $V$-genérico em $\mathbb{P}_{c}$ então $G \cap D_{n} \neq \emptyset$ para cada $n \in \omega$. Escolha $p_{n} \in G \cap D_{n}$ para cada $n \in \omega$. Como $\mu(K)=\mu(\bigcap G)$ e $p_{n} \in G$ para cada $n \in \omega$ então $\mu(K) \leq \mu\left(p_{n}\right)$ para cada $n \in \omega$. Portanto, $\mu(K) \leq c+\frac{1}{n+1}$ para cada $n \in \omega$. Logo, $\mu(K) \leq c$.

- $\mu(K) \geq c$ : Como $\left|\mathbb{P}_{c}\right|=\aleph_{0}$ e $G \subseteq \mathbb{P}_{c}$ então $|G| \leq \aleph_{0}$. Seja $\left\{P_{n}: n \in \omega\right\}$ uma enumeração sem repetições de $G$. Defina $Q_{n}=\bigcap_{m \leq n} P_{m}$ para cada $n \in \omega$. Como $P_{n} \in G$ para cada $n \in \omega$ e $G$ é um filtro então $Q_{n} \in G$ para cada $n \in \omega$. Observe que $\bigcap G=\bigcap_{n \in \omega} Q_{n}$. Logo, temos que:

$$
\mu(K)=\mu(\bigcap G)
$$




$$
\begin{aligned}
& =\mu\left(\bigcap_{n \in \omega} Q_{n}\right) \\
& =\lim _{n \in \omega} \mu\left(Q_{n}\right) .
\end{aligned}
$$

Como $Q_{n} \in G$ para cada $n \in \omega$ então $\mu\left(Q_{n}\right)>c$ para cada $n \in \omega$. Logo, $\lim _{n \in \omega} \mu\left(Q_{n}\right) \geq c$. Como $\mu(K)=\lim _{n \in \omega} \mu\left(Q_{n}\right)$ então $\mu(K) \geq c$.

O resultado abaixo nos mostra que cada translação do compacto determinado por um fitro genérico que é feita por um elemento do círculo na extenção encontra o círculo do modelo base de maneira enumerável.

Teorema 5.2.22. Fixe $c \in[0,1[$ arbitrário. Sejam $V$ um modelo transitivo enumerável de $Z F C$ tal que $\mathbb{P}_{c} \in V$ e $G$ um filtro $V$-genérico em $\mathbb{P}_{c}$. Se $K$ é o conjunto compacto determinado por $G$ então temos que:

$$
V[G] \models(K+x) \cap \mathbb{K}^{V} \text { é enumerável para cada } x \in \mathbb{K}^{V[G]} \text {. }
$$

Demonstração. Trabalhando em $V[G]$ : Suponha, por absurdo, que existe $x^{\prime} \in \mathbb{K}^{V[G]}$ tal que $\left(K+x^{\prime}\right) \cap \mathbb{K}^{V}$ é não-enumerável. Denote por $X$ o conjunto $\left(K+x^{\prime}\right) \cap \mathbb{K}^{V}$.

Iremos demonstrar que existe $\tilde{X} \subseteq X$ tal que $\tilde{X} \in V$ e $V[G] \models " \tilde{X}$ é não-enumerável ". De fato, como $X \in V[G]$ então existe $\sigma$ um $\mathbb{P}_{c}$-nome tal que $X=v_{a l}(\sigma)$. Além disso, $V[G] \models$ " $X$ é não-enumerável". Defina $X_{q}=\{x \in \mathbb{K} \cap V: q \Vdash \hat{x} \in \sigma\}$ para cada $q \in G$. Note que $\{x \in \mathbb{K} \cap V: q \Vdash \hat{x} \in \sigma\}=\left\{x \in \mathbb{K} \cap V:\left(q \Vdash^{\star} \hat{x} \in \sigma\right)^{V}\right\}$ para cada $q \in G$. Como $\left\{x \in \mathbb{K} \cap V:\left(q \Vdash^{\star} \hat{x} \in \sigma\right)^{V}\right\} \in V$ para cada $q \in G$ então $X_{q} \in V$ para cada $q \in G$.

Como $X \subseteq V$, sabemos que se $V[G] \models y \in X$ então existe $q \in G$ tal que $q \Vdash \hat{y} \in \sigma$. Então $V[G] \models X=\bigcup_{q \in G} X_{q}$. Como $G \subseteq \mathbb{P}_{c}$ e $\mathbb{P}_{c}$ é enumerável então $G$ é enumerável. Logo, existe $r \in G$ tal que $V[G] \models " X_{r}$ é não-enumerável". Basta então tomar $\widetilde{X}=X_{r}$.

Podemos supor que $X \in V$. Considere $\zeta$ um $\mathbb{P}_{c^{-}}$-nome tal que $x^{\prime}=v_{a l}(\zeta)$. Como $V[G] \models X \subseteq\left(K+x^{\prime}\right)$ então existe $p \in G$ tal que $p \Vdash \hat{X} \subseteq \rho+\xi$, sendo que $\rho$ é um $\mathbb{P}_{c}$-nome tal que $\operatorname{val}_{G}(\rho)=K$. Seja $C$ a constante dada pelo Teorema 5.2.9.

Existe $n \geq 2$ tal que $\frac{2 \cdot C \cdot \log (n)}{n}<\mu(p)-c$. De fato, $p \in G$ e $G \subseteq \mathbb{P}_{c}$ então $p \in \mathbb{P}_{c}$. Logo, $\mu(p)>c$. Então $\mu(p)-c>0$. Como $\frac{\log (n)}{n} \stackrel{n \rightarrow \infty}{\longrightarrow} 0$ e $2 \cdot C$ é uma constante então 
$\frac{2 \cdot C \cdot \log (n)}{n} \stackrel{n \rightarrow \infty}{\longrightarrow} 0$. Portanto, existe $m_{0} \in \omega$ tal que $\frac{2 \cdot C \cdot \log (m)}{m}<\mu(p)-c$ para cada $\stackrel{n}{m} \geq m_{0}$. Considerando $n \geq \max \left\{m_{0}, 2\right\}$ temos o resultado desejado.

Sabemos que $X$ é não-enumerável. Em particular, existe $X^{*} \subseteq X$ tal que $\left|X^{*}\right|=n$.

Trabalhando em $V$ : Como $X \in V, X^{*} \subseteq X$ e $\left|X^{*}\right|<\omega$ então temos $X^{*} \in V$. Por hipótese, temos que $\left|X^{*}\right|=n \geq 2$. Como $X^{*} \subseteq \mathbb{K}$ e $X^{*}$ é finito então pela Proposição 5.2 .13 temos que existe $p_{0} \subseteq \mathbb{K}$ tal que:

$$
\text { (*) } \quad X^{*} \nsubseteq p_{0}+x \text { para cada } x \in \mathbb{K}^{V} \text {. }
$$

Adicionalmente, a Proposição 5.2 .13 nos fornece a desigualdade $\mu\left(p_{0}\right) \geq 1-\frac{2 \cdot C \cdot \log (n)}{n}$ com $p_{0}$ sendo reunião finita de intervalos fechados com extremos racionais.

Demonstraremos que $p_{0}$ é uma condição de $\mathbb{P}_{c}$. Note que basta mostrarmos que $\mu\left(p_{0}\right)>c$. De fato, temos que:

$$
\begin{aligned}
\mu\left(p_{0}\right) \geq 1-\frac{2 \cdot C \cdot \log (n)}{n} \Longleftrightarrow & \mu\left(p_{0}\right)-1 \geq-\frac{2 \cdot C \cdot \log (n)}{n} ; \\
& \Longleftrightarrow 1-\mu\left(p_{0}\right) \leq \frac{2 \cdot C \cdot \log (n)}{n} ; \\
& \Longrightarrow 1-\mu\left(p_{0}\right)<\mu(p)-c .
\end{aligned}
$$

Portanto, temos que $\mu\left(p_{0}\right)>c+(1-\mu(p))$. Como $\left.\mu(p) \in\right] c, 1[$ temos que $1-\mu(p)>0$. Logo, $\mu\left(p_{0}\right)>c$.

Defina $p_{1}=p_{0} \cap p$. Temos que $p_{1}$ é uma condição de $\mathbb{P}_{c}$. De fato, $p_{0}$ é uma reunião finita de intervalos fechados com extremos racionais e $p$ é uma reunião finita de intervalos fechados com extremos racionais então $p_{1}$ é uma reunião finita de intervalos fechados com extremos racionais. Temos a seguinte desigualdade:

$$
\mu(p)>c+\frac{2 \cdot C \cdot \log (n)}{n} .
$$

Além disso, temos:

$$
\mu\left(p_{0}\right) \geq 1-\frac{2 \cdot C \cdot \log (n)}{n} .
$$

Somando as desigualdades e temos: 
○

$$
\mu\left(p_{0}+\mu(p)>1+c .\right.
$$

Logo, temos que:

$$
\begin{aligned}
\mu\left(p_{1}\right) & =\mu\left(p_{0} \cap p\right) \\
& =\mu\left(p_{0}\right)+\mu(p)-\mu\left(p_{0} \cup p\right) \\
& >c+\left(1-\mu\left(p_{0} \cup p\right)\right) .
\end{aligned}
$$

Como $\left.\left.\mu\left(p_{0} \cup p\right) \in\right] c, 1\right]$ então $1-\mu\left(p_{0} \cup p\right) \geq 0$. Portanto, temos que $\mu\left(p_{1}\right)>c$.

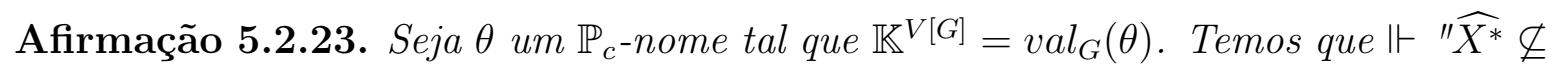
$p_{1}+x$ para cada $x \in \theta$."

Demonstração. Sabemos que $X^{*}$ é um conjunto finito. Seja $\left\{x_{0}, x_{1}, \ldots, x_{n}\right\}$ uma enumeração sem repetições de $X^{*}$. Por $(*)$ temos que $V \models " X^{*}+x \nsubseteq p_{1}$ para cada $x \in \mathbb{K}^{V}$ ". Para demonstrarmos o resultado desejado iremos proceder por contradição. Suponha por absurdo que existe $q \in \mathbb{P}$ tal que $q \not \forall " X^{*} \nsubseteq p_{1}+x$ para cada $x \in \theta^{\prime}$. Então existe $H$ filtro $V$-genérico em $\mathbb{P}_{c}$ com $q \in H$ tal que $V[H] \not X^{*} \nsubseteq p_{1}+x$ para cada

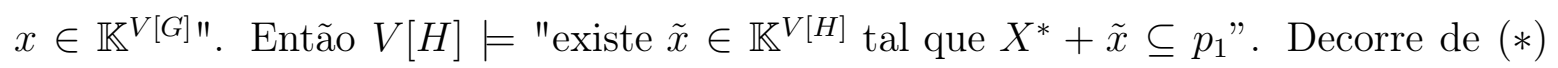
que $\tilde{x} \notin V$.

Como $p_{1} \in \mathbb{P}_{c}$ (Afirmação 3.1.4) então existem $n \in \omega$ e números racionais $a_{0}, b_{0}, a_{1}, b_{1}, \ldots, a_{n}, b_{n}$ tais que $p_{1}=\bigcup_{j=0}^{k}\left[a_{j}, b_{j}\right]$. Logo, para cada $y \in \mathbb{K}^{V}$ temos que $y \in p_{1}$ se e somente se existe $j \in\{0,1, \ldots, k\}$ tal que $y \in\left[a_{j}, b_{j}\right]$. Então temos que para cada $i \in\{0,1, \ldots, n\}$ existe $j_{i} \in\{0,1, \ldots, k\}$ tal que $a_{j_{i}}<x_{i}+\tilde{x}<b_{j_{i}}$. Como $X \in V$ então $x_{i} \in V$ para cada $i \in\{0,1, \ldots, n\}$. Observe que $a_{j}, b_{j} \in V$ para cada $j \in\{0,1, \ldots, k\}$. Seja $\left.\delta \in\right] 0,1[$ tal que $] \tilde{x}-\delta, \tilde{x}+\delta[\subseteq] a_{j_{i}}-x_{i}, b_{j_{i}}+x_{i}\left[\right.$ para cada $i \in\{0,1, \ldots, n\}$. Como $\mathbb{Q}^{V[G]} \cap \mathbb{K}^{V[G]}$ é denso em $\mathbb{K}^{V[G]}$ então existe um número racional $z \in \mathbb{K}^{V}$ tal que $\left.z \in\right] \tilde{x}-\delta, \tilde{x}+\delta[$. Observe que $\mathbb{Q}^{V}=\mathbb{Q}^{V[G]}$. Logo, temos que $V \models X^{*}+z \subseteq p_{1}$. Contradição.

Afirmação 5.2.24. Existe $p_{2} \in \mathbb{P}_{c}$ tal que $p_{2} \leq p_{1}$ e $p_{2} \Vdash \widehat{X^{*}} \nsubseteq \rho+\xi$.

Demonstração. Considere $G^{\prime}$ filtro $V$-genérico em $\mathbb{P}_{c}$ com $p_{1} \in G^{\prime}$. Como $\Vdash " \forall x \in$ $\theta\left(\widehat{X^{*}} \nsubseteq p_{1}+x\right)$ "então temos que $V\left[G^{\prime}\right] \models " \forall x \in \mathbb{K}\left(X^{*}+x \nsubseteq p_{1}\right)$ ". Logo, temos que $V\left[G^{\prime}\right] \models " \forall x \in \mathbb{K}\left(X^{*}+x \nsubseteq K\right)$ ". Como $V\left[G^{\prime}\right] \models " \forall x \in \mathbb{K}\left(X^{*}+x \nsubseteq K\right)$ "então 
existe $q \in G^{\prime}$ tal que $q \Vdash " \forall x \in \theta\left(\widehat{X^{*}} \nsubseteq \rho+x\right)$ ". Defina $p_{2}=q \cap p_{1}$. Como $G^{\prime} \subseteq \mathbb{P}_{c}$ é um filtro então $p_{2} \in G^{\prime}$. Como $p_{2} \leq q$ e $q \Vdash " \forall x \in \theta\left(\widehat{X^{*}} \nsubseteq \subseteq \rho+x\right)$ "então $p_{2} \Vdash$ " $\forall x \in \theta\left(\widehat{X^{*}} \nsubseteq \rho+x\right)$ ". Particularmente, $p_{2} \Vdash " \widehat{X^{*}} \nsubseteq \rho+\xi^{\prime}$.

Afirmamos que $p_{2} \Vdash " \widehat{X^{*}} \subseteq \rho+\xi^{\prime}$. De fato, sabemos que $p \Vdash " \widehat{X} \subseteq \rho+\xi^{\prime}$. Então $p_{2} \Vdash " \widehat{X} \subseteq \rho+\xi^{\prime}$, pois $p_{2}$ estende $p$. Como $V \models X^{*} \subseteq X$ então $\Vdash \widehat{X^{*}} \subseteq \widehat{X}$. Logo, $p_{2} \Vdash " \widehat{X^{*}} \subseteq \rho+\xi "$. Contradição.

Abaixo a apresentação de que existe um modelo que satisfaz a Conjectura Dual de Borel em $\mathbb{K}$ e, portanto, na reta real.

Teorema 5.2.25. Sejam $V$ um modelo transitivo enumerável de $Z F C$ tal que $\mathbb{P}=$ $\left(\right.$ Func $\left._{\omega}\left(\omega_{2}^{V} \times \omega, 2\right), \supset\right) \in V$ e $G$ um filtro $V$-genérico em $\mathbb{P}$. Então temos que:

$$
V[G] \models D B C_{\mathbb{K}}
$$

Demonstração. Iremos proceder por contradição. Suponha por absurdo que a Conjectura Dual de Borel não vale em $V[G]$. Trabalhando em $V[G]$ podemos encontrar $X \subseteq \mathbb{K}$ um conjunto fortemente magro tal que $|X|=\aleph_{1}$.

Usando a notação da Definição 2.3.12 considere $\left(Q_{\xi}, \leq_{Q_{\xi}}\right)=\left(F u n c_{\omega}(\omega, 2), \supset\right)$ para cada $\xi<\omega_{2}^{V}$. Pelo Lema 2.3 .13 temos que existe $\zeta<\omega_{2}^{V}$ tal que $X \in V\left[G_{\zeta}\right]$, onde $G_{\zeta}=G \cap \mathbb{P}_{\zeta}$

Fixe $c \in \mathbb{K}^{V}$ um número racional. Observe que:

$$
\begin{gathered}
\operatorname{Func}_{\omega}\left(\omega_{2}^{V} \times \omega, 2\right) \cong \operatorname{Func}_{\omega}\left(\left(\omega_{2}^{V} \backslash\{\zeta\}\right) \times \omega, 2\right) \times \operatorname{Func}_{\omega}(\{\zeta\} \times \omega, 2) \\
\cong \operatorname{Func}_{\omega}\left(\left(\omega_{2}^{V} \backslash\{\zeta\}\right) \times \omega, 2\right) \times \operatorname{Func}_{\omega}(\omega, 2) \\
\cong \operatorname{Func}_{\omega}\left(\left(\omega_{2}^{V} \backslash\{\zeta\}\right) \times \omega, 2\right) \times \mathbb{P}_{c} .
\end{gathered}
$$

Defina $\mathbb{P}^{\prime}=$ Func $_{\omega}\left(\left(\omega_{2}^{V} \backslash\{\zeta\} \times \omega\right), 2\right)$. Note que $\mathbb{P} \cong \mathbb{P}^{\prime} \times \mathbb{P}_{c}$. Seja $\psi: \mathbb{P} \longrightarrow \mathbb{P}^{\prime} \times \mathbb{P}_{c}$ um isomorfismo de ordem. Como $\psi[G] \subseteq \mathbb{P}^{\prime} \times \mathbb{P}_{c}$ é um filtro em $\mathbb{P}^{\prime} \times \mathbb{P}_{c}$ então existem $G^{\prime} \subseteq \mathbb{P}^{\prime}$ e $G_{c} \subseteq \mathbb{P}_{c}$ filtros em $\mathbb{P}^{\prime}$ e $\mathbb{P}_{c}$ respectivamente tais que $\psi[G]=G^{\prime} \times G_{c}$. Como $\psi[G]$ é $V$-genérico pelo Lema do Produto (Teorema 2.3.10) temos que $V[G]=V\left[G^{\prime}\right]\left[G_{c}\right]$.

Afirmação 5.2.26. $X \in V\left[G^{\prime}\right]$. 
Demonstração. Como $\psi[G]$ é um filtro $V$-genérico pelo Lema do Produto (Teorema 2.3 .10 temos que $G^{\prime}$ é um filtro $V$-genérico em $\mathbb{P}^{\prime}$. Sabemos que:

$$
\left.\left.\mathbb{P}^{\prime} \cong\left(F u n c_{\omega}(\zeta \times \omega), 2\right) \times F u n c_{\omega}\left(\omega_{2}^{V} \backslash(\zeta+1)\right) \times \omega, 2\right)\right)
$$

Então existem $G_{A} \subseteq F u n c_{\omega}(\zeta \times \omega, 2)$ e $G_{B} \subseteq F u n c_{\omega}\left(\omega_{2}^{V} \backslash(\zeta+1) \times \omega, 2\right)$ filtros em $\operatorname{Func}_{\omega}(\zeta \times \omega, 2)$ e $\operatorname{Func}_{\omega}\left(\omega_{2}^{V} \backslash(\zeta+1) \times \omega, 2\right)$ respectivamente tais que $\psi[G]=G_{A} \times G_{B}$. Como $G^{\prime} \subseteq \mathbb{P}^{\prime}$ é um filtro $V$-genérico em $\mathbb{P}^{\prime}$ então pelo Lema do Produto (Teorema 2.3.10 temos que $V\left[G^{\prime}\right]=V\left[G_{A}\right]\left[G_{B}\right]$. Pela Observação 2.3.11 temos que $G_{A}=G_{\zeta}=$ $\psi[G] \cap \mathbb{P}_{\zeta}$. Como $X \in V\left[G_{\zeta}\right]$ e $V\left[G_{\zeta}\right] \subseteq V\left[G_{\zeta}\right]\left[G_{B}\right]$ então $X \in V\left[G_{A}\right]\left[G_{B}\right]$. Logo, $X \in V\left[G^{\prime}\right]$, pois $V\left[G^{\prime}\right]=V\left[G_{A}\right]\left[G_{B}\right]$.

Aplicação do Teorema 5.2.22 : Fixe $c \in\left[0,1\left[^{V}\right.\right.$ um número racional arbitrário. Pelo Lema 5.2 .18 temos que $\mathbb{P}_{c}$ e $F u n c_{\omega}(\omega, 2)$ produzem as mesmas extensões genéricas. Pelo Lema do Produto (Teorema 2.3.10 temos que $G_{c}$ é um filtro $V\left[G^{\prime}\right]$-genérico em $\mathbb{P}_{c}$. Portanto, o filtro $G_{c}$ adiciona apenas um real de Cohen na extensão genérica $V\left[G^{\prime}\right]\left[G_{c}\right]$. Como $\mathbb{P}_{c} \in V$ e $V \subseteq V\left[G^{\prime}\right]$ então $\mathbb{P}_{c} \in V\left[G^{\prime}\right]$. Denote por $K_{c}$ o conjunto compacto determinado por $G_{c}$. Pela Proposição 5.2 .20 temos que $V\left[G^{\prime}\right]\left[G_{c}\right] \models " \mu\left(K_{c}\right)=c^{\prime}$. Pelo Teorema 5.2 .22 temos que:

$$
V\left[G^{\prime}\right]\left[G_{c}\right] \models \forall x \in \mathbb{K}^{V\left[G^{\prime}\right]\left[G_{c}\right]}\left(\left|\left(K_{c}+x\right) \cap \mathbb{K}^{V\left[G^{\prime}\right]}\right| \leq \aleph_{0}\right) .
$$

Pela Afirmação 5.2 .26 temos que $X \subseteq \mathbb{K}^{V\left[G^{\prime}\right]}$. Em particular, temos:

$$
V\left[G^{\prime}\right]\left[G_{c}\right] \models \forall x \in \mathbb{K}^{V\left[G^{\prime}\right]\left[G_{c}\right]}\left(\left|\left(K_{c}+x\right) \cap X\right| \leq \aleph_{0}\right) .
$$

Como $V[G]=V\left[G^{\prime}\right]\left[G_{c}\right]$ então para cada número racional $c \in[0,1[V$ temos que:

$$
V[G] \models \forall x \in \mathbb{K}^{V\left[G^{\prime}\right]\left[G_{c}\right]}\left(\left|\left(K_{c}+x\right) \cap X\right| \leq \aleph_{0}\right) \wedge \mu\left(K_{c}\right)=c .
$$

Afirmação 5.2.27. $V[G] \models \exists K \subseteq \mathbb{K}^{V\left[G^{\prime}\right]}\left(\mu(K)=1 \wedge \forall x \in \mathbb{K}^{V[G]}\left(|(K+x) \cap X| \leq \aleph_{0}\right)\right.$.

Demonstração. Defina $K=\bigcup_{c \in \mathbb{Q} \cap \mathbb{K}^{V}} K_{c} \cdot \operatorname{Em} V[G]$, temos:

$$
\mu(K)=\mu\left(\bigcup_{c \in \mathbb{Q} \cap \mathbb{K}^{V}} K_{c}\right)
$$




$$
\begin{aligned}
& \geq \sup \left\{\mu\left(K_{c}\right): c \in \mathbb{Q} \cap \mathbb{K}^{V}\right\} \\
& =\sup \left\{c: c \in \mathbb{Q} \cap \mathbb{K}^{V}\right\} \\
& =1 .
\end{aligned}
$$

Como $\left|\left(K_{c}+x\right) \cap X\right| \leq \aleph_{0}$ para cada $x \in \mathbb{K}^{V[G]}$ e para cada $c \in \mathbb{Q} \cap[0,1[V$ então temos que $|(K+x) \cap X| \leq \aleph_{0}$ para cada $x \in \mathbb{K}^{V[G]}$.

Pela Afirmação 5.2 .27 temos que o complementar de $K$ em $\mathbb{K}^{V\left[G^{\prime}\right]}$ atesta que $X$ não é fortemente magro em $V[G]$. Contradição. 


\section{Bibliografia}

[1] Arnold W. Miller. Corretions ans additions to: "Some properties of measure and category". Transations of the American Mathematical Society, 271(1): 347-348, 1982.

[2] Arnold W. Miller. The $\gamma$-Borel Conjecture. Arch. Math. Logic 44, 425-434 (2005).

[3] Arnold W. Miller e David H. Fremlin. On some properties of Hurewicz, Menger and Rothberger. Fundamenta Mathematicae, 129(1): 17-33, 1988.

[4] E. Borel. Sur la classification des ensambles de mesure nulle. Bull. Soc. Math.France, 47 (1919) 97-125.

[5] T. J. Carlson, Strong measure zero and strongly meager sets, Proceedings of the American Mathematical Society 118:2 (1993), 577-586.

[6] Fred Galvin, Jan Mycielski e Robert Solovay. Strong Measure Sets. Notices of American Mathematical Society, página A-280 1973.

[7] Fred Galvin, Jan Mycielski e Robert Solovay. Strong measure zero and infinite games, Arch. Math. Logic (2017) 56, 725-732.

[8] Martin Goldstern, Haim Judah e Saharon Shelah. Strong measure zero sets without Cohen reals. The Journal of Symbolic Logic, 58(4): 1323-1341, 1993.

[9] H. Judah, T. Bartoszyński, Set Theory On The structure of the Real Line, AK Peters/CRC Press, Weslley, Massachusetts, QA248:B374 (1995), 511.3'22-dc20.

[10] K. Kuratowski. Topology, volume II. London; Polish Scientific Publishers, Warsaw, 1968.

[11] G.G. Lorentz. On a Problem of Additive Number Theory. Proc. Amer. Math. Soc. 5 (1954), 838-841.

[12] Richard Laver. On the Consistency of Borel's Conjecture. Acta-Math., 137(3-4): 151-169, 1976. 
[13] Fritz Rothberger. Eine aquivalenz zwishen der kontinuumhypothese under der Existenz der Lusinschen und Sierpinschishen mengen. Fundamenta Mathematicae, 30, 215-217, 1938.

[14] Fritz Rothberger. Sur les familles indenombrables de suites de nombres naturels et les problemes concernant la propriete C. Procedings of the Cambridge Philosophical Society, 37: 109-126, 1941.

[15] W.Sierpiński. Sur un ensemble non dénombrable, dont toute image continue est de mesure nulle. Fund. Math. 11 (1928), 302-304. 